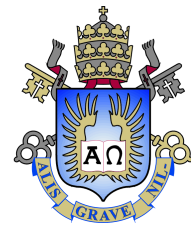

Monique Feitosa Dali

\title{
Flow in porous media with macropores using
} Brinkman's model

Dissertation presented to the Programa de Pós-Graduação em Engenharia Mecânica of PUC-Rio in partial fulfillment of the requirements for the degree of Mestre em Engenharia Mecânica.

Advisor : Prof. Márcio da Silveira Carvalho Co-advisor: Dr. Frederico Carvalho Gomes 


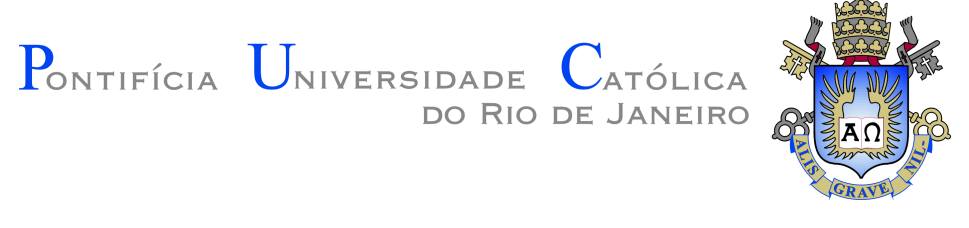

Monique Feitosa Dali

\section{Flow in porous media with macropores using \\ Brinkman's model}

Dissertation presented to the Programa de Pós-Graduação em Engenharia Mecânica of PUC-Rio in partial fulfillment of the requirements for the degree of Mestre em Engenharia Mecânica. Approved by the Examination Committee.

Prof. Márcio da Silveira Carvalho

Advisor

Departamento de Engenharia Mecânica - PUC-Rio

Dr. Frederico Carvalho Gomes

Co-advisor

Departamento de Engenharia Mecânica - PUC-Rio/LMMP

Prof. Abelardo Borges Barreto Junior

Departamento de Engenharia Mecânica - PUC-Rio

Dr. Leonardo Cabral Pereira

Petrobras - CENPES

Rio de Janeiro, April 02nd, 2019 
All rights reserved.

\section{Monique Feitosa Dali}

Bachelor in Industrial Engineering by Universidade Federal Fluminense (2014).

Bibliographic data

Dali, Monique Feitosa

Flow in porous media with macropores using Brinkman's model / Monique Feitosa Dali; advisor: Márcio da Silveira Carvalho; co-advisor: Frederico Carvalho Gomes. - 2019.

107 f: il.color. ; $30 \mathrm{~cm}$

Dissertação (mestrado) - Pontifícia Universidade Católica do Rio de Janeiro, Departamento de Engenharia Mecânica, 2019 .

Inclui bibliografia

1. Engenharia Mecânica - Teses. 2. Equação de Brinkman;. 3. Método dos elementos finitos;. 4. Permeabilidade equivalente;. 5. Meios porosos carstificados.. I. Carvalho, Márcio da Silveira. II. Gomes, Frederico Carvalho. III. Pontifícia Universidade Católica do Rio de Janeiro. Departamento de Engenharia Mecânica. IV. Título. 


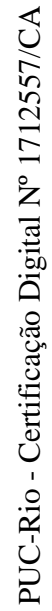

To my big family for their love and support. 


\section{Acknowledgments}

I would like to thank my entire family who has always supported, guided and helped me at all times. In particular, to my grandparents Maria Francisca, Moacyr Feitosa and Ismeria Maria for their dedication and my husband André Dali for his support.

To all my university colleagues, especially my friends, Gabriela Castro and Paulo Roberto, for personal and professional partnership.

To my advisor, Prof. Márcio Carvalho for the shared knowledge and dedication to lead me in this project.

To my lab colleagues from LMMP, for the work environment, all the questions and support, and Frederico Gomes for his attention.

To Coordenação de Aperfeicoamento de Pessoal de Nível Superior (CAPES), Fundação Carlos Chagas Filho de Amparo à Pesquisa do Estado do Rio de Janeiro (FAPERJ) and PUC-Rio for the scholarship.

This study was financed in part by the Coordenação de Aperfeiçoamento de Pessoal de Nível Superior - Brasil (CAPES) - Finance Code 001, Fundação Carlos Chagas Filho de Amparo à Pesquisa do Estado do Rio de Janeiro (FAPERJ, E-26/200.696/2018) and Petrobras.

Finally, I appreciate I have achieved one more dream in my life. 


\section{Abstract}

Dali, Monique Feitosa; Carvalho, Márcio da Silveira (Advisor); Gomes, Frederico Carvalho (Co-Advisor). Flow in porous media with macropores using Brinkman's model. Rio de Janeiro, 2019. 107p. Dissertação de Mestrado - Departamento de Engenharia Mecânica, Pontifícia Universidade Católica do Rio de Janeiro.

The present work focuses on a numerical investigation of the flow through porous media with macropores, such as carbonates. The presence of vugs and fractures have a strong effect on the flow characteristics. The flow through the porous matrix is usually described by the Darcy equation and the flow through the macropores by Stokes equation. The coupling between these two distinct approaches brings great complexity to the modeling of such flows. In this work, we use Brinkman formulation that is able to describe the flow, both in the porous matrix and macropores with a single differential equation. We solved the set of differential equations using the finite element model and implemented the code in the FEniCS platform. We first solved the 1-D flow through parallel plates with one of the walls being a porous material. The goal was to compare the predictions obtained with the Brinkman formulation to that obtained by using the Beavers-Joseph boundary condition. Then, we solved a 2-D flow through a porous medium with macropores. The geometry of the pore structure was obtained from $2 \mathrm{D}$ slices of tomographic images of carbonates. The goal of this analysis was to evaluate an equivalent permeability as a function of macropores area and structures.

\section{Keywords}

Brinkman Equation; Finite Element Method; Equivalent permeability; Karst porous media. 


\section{Resumo}

Dali, Monique Feitosa; Carvalho, Márcio da Silveira; Gomes, Frederico Carvalho. Escoamento em meios porosos com macroporos utilizando o modelo de Brinkman. Rio de Janeiro, 2019. 107p. Dissertação de Mestrado - Departamento de Engenharia Mecânica, Pontifícia Universidade Católica do Rio de Janeiro.

O presente trabalho foca em uma investigação numérica do escoamento através de meios porosos com macroporos, como os carbonatos. A presença de vugs e fraturas tem um forte efeito na característica do escoamento. $\mathrm{O}$ fluxo através da matriz porosa é geralmente descrito pela equação de Darcy e o fluxo através dos macroporos pela equação de Stokes. O acoplamento entre essas duas abordagens distintas traz grande complexidade para a modelagem de tais fluxos. Neste trabalho, utilizamos a formulação de Brinkman que é capaz de descrever ambos os fluxos através da matriz porosa e macroporos com uma única equação diferencial. Resolvemos o sistema de equações diferenciais usando o modelo de elementos finitos e implementamos o código com auxílio da plataforma FEniCS. Primeiro, resolvemos o fluxo 1-D através de placas paralelas com uma das paredes sendo um material poroso. O objetivo foi comparar as previsões obtidas pela formulação de Brinkman com aquela obtida usando a condição de contorno de BeaversJoseph. Então, resolvemos um fluxo 2-D através de um meio poroso com macroporos. A geometria da estrutura porosa foi obtida a partir de cortes 2D de imagens tomográficas de carbonatos. O objetivo desta análise foi avaliar uma permeabilidade equivalente em função da área e estrutura dos macroporos.

\section{Palavras-chave}

Equação de Brinkman; Método dos elementos finitos; Permeabilidade equivalente; Meios porosos carstificados. 


\section{Table of contents}

1 Introduction $\quad 16$

1.1 Problem Statement 16

$\begin{array}{ll}1.2 \text { Motivation } & 16\end{array}$

$\begin{array}{lll}1.3 & \text { Dissertation goals } & 17\end{array}$

$\begin{array}{lll}1.4 & \text { State of art } & 17\end{array}$

1.5 Delimitation of the study 22

1.6 Division of Chapters 22

2 Literature Review $\quad 24$

2.1 Porous Media Flow 24

2.2 Carbonate Rocks 25

2.2.1 Finite element method 27

3 Methodology $\quad 29$

3.1 Mathematical modeling 29

$\begin{array}{lll}3.1 .1 & \text { Conservations equations } & 29\end{array}$

3.1.2 Conservation of linear momentum 30

3.1.2.1 Navier-Stokes Equations 30

3.1.2.2 Darcy's Law 30

3.1.2.3 Brinkman Equation 31

3.2 Numerical formulation 32

3.2.1 FEniCS 32

3.2.2 Brinkman's variational formulation 33

4 Model analysis $\quad 36$

4.1 Flow behavior near a porous solid and channel interface 36

4.1.1 Mesh test 37

$\begin{array}{ll}\text { 4.2 Flow behavior at solid/void interface } & 39\end{array}$

4.2.1 The effect of aperture 43

4.2.2 Comparison Beavers-Joseph boundary condition 48

4.2.3 Effect of model parameter $\mu^{*}$ in the flow prediction 49

5 Flow through vuggy porous media $\quad 53$

5.1 Core Samples 53

5.1.1 Model of a rock sample $\quad 55$

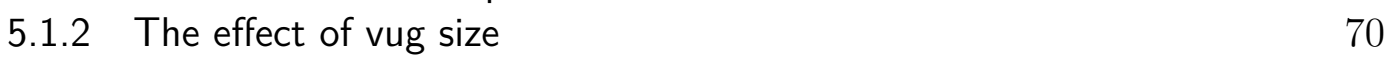

$\begin{array}{lll}5.1 .3 & \text { Results analysis } & 83\end{array}$

$\begin{array}{lll}6 & \text { Discussion and conclusions } & \mathbf{9 0}\end{array}$

$\begin{array}{lll}6.1 & \text { Future works } & 91\end{array}$

$\begin{array}{ll}\text { Bibliography } & 93\end{array}$

$\begin{array}{lll}\text { A FEniCS Algorithms } & 98\end{array}$ 


\section{List of figures}

Figure 2.1 Permeability combinations. From Exploration and Production Geology.

Figure 2.2 Dunham (1967) classification for carbonate sedimentary rocks with Embry \& Klovan (1971) modifications. From Nichols, 1999.

Figure 2.3 Porosity types usually found in carbonates. From Choquette \& Pray, 1970.

Figure 2.4 Steps of Finite Element Analysis

Figure 3.1 Brinkman's model. Adapted from Choquette \& Pray, 1970. 31

Figure 3.2 Type of finite element used. From Arnold and Logg, 2014. 33

Figure 4.1 First mesh generated to be tested, where the green area is the free flow section and the brown one is the porous medium. 37

Figure 4.2 Comparison between Poiseuille and our numeric model. 39

Figure 4.3 Speed profile proposed by Beavers and Joseph. From Beavers \& Joseph, 1967

Figure 4.4 Velocity profile at the midpoint of the section $(\mathrm{x}=0.1$ $\mathrm{m})$ with multiple permeabilities input and $\Delta P=6,894.76 \mathrm{~Pa}$.

Figure 4.5 Zoom in at the interface $(\mathrm{y}=0.01 \mathrm{~m})$ between free section and permeable wall to show the slip velocity above the interface.

Figure 4.6 Velocity field for three different porous medium permeabilities input and $\Delta P=6,894.76 \mathrm{~Pa}$. Below each velocity field is the velocity profile at the midpoint of the section $(\mathrm{x}=0.1 \mathrm{~m}) .42$

Figure 4.7 Comparison of velocity profile between theoretical and experimental presented by Yao \& Huang, and numerical results of this work.

Figure 4.8 Beavers \& Joseph experimental arrangement. From Beavers \& Joseph, 1967.

Figure 4.9 Generated mesh to analyze the Beavers-Joseph condition with an smaller aperture, where the green area is the free section and the other is the porous medium.

Figure 4.10 Velocity and pressure field for $0.0008 \mathrm{~m}$ aperture with $k_{p m}=1.98 \times 10^{-9} m^{2}$ and $\Delta P=6,894.76 \mathrm{~Pa}$. On top, 4.10a, are the velocity diagram and profile at $\mathrm{x}=0.1 \mathrm{~m}$. Below this is the pressure diagram and pressure distribution from an inlet to the outlet at $\mathrm{y}=0.01 \mathrm{~m}$.

Figure 4.11 Velocity diagram and profile with same velocity scale for two heights of aperture and $\Delta P=6,894.76 \mathrm{~Pa}$.

Figure $4.12 \Phi$ as a function of $h / \sqrt{k_{p m}}$ for the same permeabilities of porous specimens used by Beavers-Joseph. $-k_{p m}=8.19 \mathrm{x}$ $10^{-8} m^{2} ; \mathbf{\Delta}, k_{p m}=3.94 \times 10^{-8} ; \mathbf{\square}, k_{p m}=9.68 \times 10^{-9} m^{2} ; \bullet, k_{p m}$ $=1.6 \times 10^{-9} m^{2}$ and $k_{p m}=6.45 \times 10^{-10} m^{2}$. 
Figure $4.13 \Phi$ as a function of $h / \sqrt{k_{p m}}$. C, $k_{p m}=8.19 \times 10^{-8} m^{2} ; \mathrm{B}$, $k_{p m}=3.94 \times 10^{-8} ; \mathrm{A}, k_{p m}=9.68 \times 10^{-9} m^{2} ; \mathrm{D}, k_{p m}=1.6 \mathrm{x}$ $10^{-9} m^{2}$ and $k_{p m}=6.45 \times 10^{-10} m^{2}$. From Beavers \& Joseph, 1967. 48

Figure 4.14 Curve adjustment for various apertures. 50

Figure 4.15 Stability model test: Pressure. 51

Figure 4.16 Stability model test: Velocity. 52

Figure 5.1 Pore size distribution for coquinas samples. Adapted from Lima et al.,2018. 54

Figure 5.2 Construction of the virtual model for sample 1. 55

Figure 5.3 Virtual model for sample 2.

Figure 5.4 X-ray microtomography of coquine samples: 3D images of the samples. $\quad 56$

Figure 5.6 Procedure used for a 2D simulation in representative geometries based on $\mu$-tomography images. 56

Figure 5.5 X-ray microtomography of coquine samples: slices extracted at the top, middle and bottom of the rocks. $\quad 57$

$\begin{array}{lll}\text { Figure 5.7 } & \text { Flow chart of digital image processing. } & 57\end{array}$

Figure 5.8 Pre-processing of a slice of the upper part (slice 1) of sample 1.

Figure 5.9 Segmentation of a slice of the upper part (slice 1) of sample 1.

Figure 5.10 Defining the voids in a slice of the upper part (slice 1) of sample 1 .

Figure 5.11 Domains for one 2D slice of sample 1.
Figure 5.12 Zoom in one part of the mesh to show the limit between the 2 domains.

Figure 5.13 Section under investigation.

Figure 5.14 Pressure field: $\mathrm{x}$-axis and $\mathrm{y}$-axis with fluid flowing in the x-direction, $\Delta P=6,894.76 \mathrm{~Pa}$ and $k_{p m}=10^{-12} \mathrm{~m}^{2}$.

Figure 5.15 Velocity field with fluid flowing in the x-direction, $\Delta P=$ $6,894.76 \mathrm{~Pa}$ and $k_{p m}=10^{-12} \mathrm{~m}^{2}$.

Figure 5.18 Flow rate for different pressure gradient inputs for the domain shown in fig. 5.13 with $k_{p m}=10^{-12} \mathrm{~m}^{2}$ and fluid flowing in the $x$ direction.

Figure 5.16 Velocity field: y-axis with fluid flowing in the x-direction, $\Delta P=6,894.76 \mathrm{~Pa}$ and $k_{p m}=10^{-12} \mathrm{~m}^{2}$.

Figure 5.17 Velocity field: $\mathrm{x}$-axis with fluid flowing in the $\mathrm{x}$-direction, $\Delta P=6,894.76 \mathrm{~Pa}$ and $k_{p m}=10^{-12} \mathrm{~m}^{2}$.

Figure 5.22 Flow rate for different pressure gradient inputs for the domain shown in fig. 5.13 with $k_{p m}=10^{-12} \mathrm{~m}^{2}$ and fluid flowing in the y-direction.

Figure 5.19 Pressure field: $\mathrm{x}$-axis and $\mathrm{y}$-axis with fluid flowing in the y-direction, $\Delta P=6,894.76 \mathrm{~Pa}$ and $k_{p m}=10^{-12} \mathrm{~m}^{2}$.

Figure 5.20 Velocity field: $\mathrm{y}$-axis with fluid flowing in the $\mathrm{y}$-direction, $\Delta P=6,894.76 \mathrm{~Pa}$ and $k_{p m}=10^{-12} \mathrm{~m}^{2}$.

Figure 5.21 Velocity field: $\mathrm{x}$-axis with fluid flowing in the y-direction, $\Delta P=6,894.76 \mathrm{~Pa}$ and $k_{p m}=10^{-12} \mathrm{~m}^{2}$. 
Figure 5.23 Comparison of the flow rate as function of pressure gradient on both flow directions with $k_{p m}=10^{-12} \mathrm{~m}^{2}$ for the domain shown in 5.13 .

Figure 5.24 Vugs enlargement on slice 1 of sample 1. From left to right, the first image is the one which was processed to identify voids as seen in 5.10 , next to this were applied different thresholds to enlarge the voids.

Figure 5.25 Pressure and velocity field for first cells described in the domains shown in fig. 5.24 with flow in the x-direction, $\Delta P=6,894.76 \mathrm{~Pa}$ and $k_{p m}=10^{-12} \mathrm{~m}^{2}$.

Figure 5.26 Pressure and velocity field for last cells described in the domains shown in fig. 5.24 with flow in the $\mathrm{x}$-direction, $\Delta P=6,894.76 \mathrm{~Pa}$ and $k_{p m}=10^{-12} \mathrm{~m}^{2}$.

Figure 5.27 Equivalent permeability $\left(k_{e q}\right)$ of the geometries shown in fig. 5.24 with different permeabilities of porous matrix, fluid flowing in the $x$-direction and $\Delta P=6,894.76 \mathrm{~Pa}$.

Figure 5.28 Equivalent permeability $\left(k_{e q}\right)$ obtained at the outlet with flow on $x$-direction and $\Delta P=6,894.76 \mathrm{~Pa}$, divided by each porous media permeability $\left(k_{p m}\right)$ as a function of the voids of the cells shown in fig. 5.24. The dependent variable is on the logarithmic scale.

Figure 5.31 Equivalent permeability $\left(k_{e q}\right)$ obtained at the outlet with flow on $y$-direction and $\Delta P=6,894.76 \mathrm{~Pa}$, divided by each porous media permeability $\left(k_{p m}\right)$ as a function of the voids of the cells shown in fig. 5.24. The dependent variable is on the logarithmic scale.

Figure 5.29 Pressure and velocity field for first cells described in fig. 5.24 with flow in the y-direction, $\Delta P=6,894.76 \mathrm{~Pa}$ and $k_{p m}=10^{-12} \mathrm{~m}^{2}$.

Figure 5.30 Pressure and velocity field for last cells described in fig. 5.24 with flow in the y-direction, $\Delta P=6,894.76 \mathrm{~Pa}$ and $k_{p m}=10^{-12} \mathrm{~m}^{2}$.

Figure 5.32 Comparison of equivalent permeability as function of vugs area on both flow directions with $k_{p m}=10^{-12} \mathrm{~m}^{2}$ and $\Delta P=6,894.76 \mathrm{~Pa}$ for slice 1 of sample 1 .

Figure 5.33 From b-d are the vugs enlarged of slice 2 (middle part) and from $\mathrm{f}$-h, slice 3 (lower part) of sample 1 . On left column are the ones which were processed to identify voids as done from $5.24 \mathrm{a}$ to $5.24 \mathrm{~b}$, next to this were applied different thresholds to enlarge voids with same threshold methods applied in fig. 5.24.

Figure 5.34 Equivalent permeability $\left(k_{e q}\right)$ for $\Delta P=6,894.76 \mathrm{~Pa}$, divided by each porous medium permeability input $\left(k_{p m}\right)$ as a function of voids of the cells shown in fig. 5.24 for sample 1. $k_{x}$ represents permeability of the porous medium when flow guidance is on the $\mathrm{x}$-direction and $k_{y}$ is when flow guidance is on the $y$-direction. 
Figure 5.35 Vugs enlargement on slice 1 (a-d), 2 (e-h) and 3 (i-l) of sample 2. In first row are the images for slice 1, which was taken from the upper part of the core sample 2. In the left column are those that have been processed to identify voids as done from $5.24 \mathrm{a}$ to $5.24 \mathrm{~b}$, next to this was applied different thresholds to increase voids with same methods applied in fig. 5.24.

Figure 5.36 Equivalent permeability $\left(k_{e q}\right)$ for $\Delta P=6,894.76 \mathrm{~Pa}$, divided by each porous medium permeability $\left(k_{p m}\right)$ input as a function of the voids of the cells shown in fig. 5.35 for sample 2 . $k_{x}$ represents permeability of porous media when flow guidance is on the $\mathrm{x}$-direction and $k_{y}$ is when flow guidance is on the $\mathrm{y}$-direction.

Figure 5.37 Subsets from the 3-D microtomography of macropores with different porosity values: (a) $\phi=0.05$, (b) $\phi=0.075$, and (c-f) $\phi=0.10$. From Okabe \& Blunt, 2007

Figure 5.38 Variation of computed permeabilities in combined images of fig. 5.37 with different macroporosities. From Okabe \& Blunt, 2007.

Figure 5.39 Curve adjustment based on the Carman-Kozeny equation with $\Delta P=6,894.76 \mathrm{~Pa}$ and $k_{p m}=10^{-12} \mathrm{~m}^{2}$.

Figure 5.40 Representative cell of a 2D vuggy porous media. From Golfier et al., 2015.

Figure 5.41 Procedure adopted to create an equivalent vug system.

Figure 5.42 Size partitioning and number of sub-samples for a slice of sample Am05.

Figure 5.43 Equivalent homogeneous permeabilities for $\Delta P=$ 6, 894.76 Pa, different slices of core samples and $\mathbf{n}$ sub-samples.

Figure 5.44 Equivalent homogeneous permeability for macroporosity under $25 \%$.

Figure 5.45 Images used to build graphic 5.43. Figures 5.45a, 5.45b, $5.45 \mathrm{e}$ and $5.45 \mathrm{~g}$ were extracted from sample $2, \mathrm{Am} 05$. The others are images based on sample $1, \mathrm{Am08}$. 


\section{List of tables}

Table 4.1 Comparison of results for mesh refining. 37

Table 4.2 Comparison of the values obtained between Poiseuille's flow and this numerical model. 38

Table 4.3 Curve adjustment for boundary condition equation. 49

Table 4.4 Difference between methods. 51

Table 5.1 Core plug geometry details and routine core analysis results for coquinas samples.

Table 5.2 Comparison between calculated equivalent permeability $\left(k_{e q}\right)$ using eq.5-3 and equivalent permeability $\left(k_{e q}\right)$ for a $\mu C T$ image divided by porous region permeability $\left(k_{p m}\right)$. 


\section{List of Abreviations}

\section{Greek Symbols}

$\begin{array}{ll}\rho & \text { Liquid density }\left[\mathrm{kg} / \mathrm{m}^{3}\right] \\ \boldsymbol{\tau} & \text { Stress tensor }\left[\mathrm{N} / \mathrm{m}^{2}\right] \\ \boldsymbol{\mu} & \text { Viscosity }[\text { Pa.s }] \\ \boldsymbol{\mu}^{*} & \text { Effective viscosity }[\text { Pa.s }] \\ \phi & \text { Porosity } \\ \phi_{\eta} & \text { Macroporosity } \\ \Omega & \text { Space domain } \\ \alpha & \text { Correction number } \\ \Gamma & \text { Boundary domain } \\ \mu \nabla^{2} \boldsymbol{u} & \text { Viscous term }\end{array}$

\section{Roman Symbols}

BJ Beavers \& Joseph boundary condition

$\boldsymbol{u} \quad$ Velocity field $[\mathrm{m} / \mathrm{s}]$

$\mathrm{k}_{e q} \quad$ Equivalent permeability $\left[\mathrm{m}^{2}\right]$

$\mathrm{k}_{p m} \quad$ Permeability of porous matrix $\left[\mathrm{m}^{2}\right]$

$Q \quad$ Flow rate $\left[\mathrm{m}^{3} / \mathrm{s}\right]$ 
"Everybody is a genius. But if you judge a fish by its ability to climb a tree, it will live its whole life believing that it is stupid."

Unknown author . 


\section{Introduction}

\section{1}

\section{Problem Statement}

In the oil industry, the difficulty of extracting hydrocarbons increases with each new reserve found, impelling research on the required technological development. In the case of Brazil, the increase in proved reserves brought a greater challenge due to the high cost of extracting hydrocarbons from the pre-salt layer. This cost is related to the hard access to oil reservoirs and technological challenges posed by the reservoirs characteristic.

Faced with this scenario, the studies in the area seek to optimize the techniques of production. For this, it is essential to understand in advance the behavior of the reservoir over its useful life, so that better choices of production techniques and infrastructure are made.

Carbonate rocks were formed through basins decomposed by biological materials, chemicals and debris. The presence of this type of rock in reservoirs is abundant, where surveys estimate that approximately $60 \%$ of the oil reserves are in carbonates[1].

This type of rock has attributes that vary considerably in relation to other types of geological formations, being expected a significant variation in regard to the porosity and the permeability. The structure also presents particularities, contributing to its heterogeneity, being able to present caves, fractures, isolated and connected cavities[2].

\section{2}

\section{Motivation}

Permeability is a parameter of vital importance during the production of oil fields. For a particular rock type, it is also highly dependent on pore geometry, both size and the degree of interconnectivity between pores and voids. The permeability can be determined through experimental measurement and theoretical calculation[3]. There are many problems in both methods due to the spatial multi-scale of fractures and vugs in heterogeneous porous media[4]. Many available theoretical models can be used to estimate the permeability as a 
function of porosity. However, because of the high heterogeneity of carbonates, the determination of permeability in carbonates samples is still a challenge. The problem becomes even more complex because of the different pore sizes that are encountered in carbonates. The presence of vugs and fracture have a great impact on the fluid flow through carbonate rocks, and the equivalent permeability is a function of the permeability of the porous matrix (micropores) and the volume and structure of the vugs and fractures (macropores).

\section{3}

\section{Dissertation goals}

The main objective of this work is to develop a methodology based on flow simulation to estimate the permeability in heterogeneous porous media containing large vugs.

To achieve these goals, the work will be developed through the following steps:

- Implementation of simulation tool to study flow through vuggy porous media using Brinkman's model;

- Analyze the flow behavior near porous matrix-vug interface predicted by Brinkman's model;

- Parametric analysis of the flow behavior and estimated equivalent permeability as a function of vug volume (area in 2D) and structure.

The study primarily focused on evaluating whether the mathematical model adopted replicates the flow behavior at the interface between free flow and porous surface observed in experiments. To perform the study, the developed simulation tool was applied to numerically determine the equivalent permeability. Slices of two micro-tomographic carbonate samples were used to create a two-dimensional geometry composed by two sections (vugs and porous matrix). The purpose of the parametric analysis is to evaluate the geometry effects of the vug structure.

\section{4}

\section{State of art}

In carbonates, there is the presence of many free flow domains such as fractures, vugs, large pores, and cavities. In this case, the fluid flows preferentially through these open spaces, leading to behavior different than that in a heterogeneous porous media. A carbonate reservoir with large cavities is a typical medium of double-porosity due to the different sizes between the matrix pores and the vugs. 
The permeability for a given rock can be determined by experimental measurement and theoretical calculation. The second method demands to build a model that corresponds to the flow condition to predict the equivalent permeability in complex media using equivalence principles. A common porous media has a solid fluid flow theory that explain flow characteristic and numerical simulation[3].

The permeability of a heterogeneous porous media can be numerically determined by solving Navier-Stokes or Stokes equation in the pore space. In fractured-vuggy porous media, these equations described the flow in porescale (micro) and large cavities (macro). The modeling with different scales of investigation leads to an extremely expensive computational cost.

The Two-Domain Approach (TDA) uses a mathematical model in the two different flow regions, which are coupled by specific interface conditions. A classical way in TDA is to solve flow in karst reservoirs or permeable surfaces using Navier-Stokes to solve the equation in the macropores and Darcy's Law to solve flow in the porous solid matrix. The coupling between the two domains demands explicitly interface modeling, which is usually done by the BeaversJoseph[5] equation.

The Beavers-Joseph (BJ) boundary condition (Eq.1-1) proposes a model to describe the flow behavior at the interface between free-flow and permeable walls. The problem of defining relevant boundary conditions at the interface between the two domains then remains.

$$
-\left.\frac{d u}{d y}\right|_{\text {int }}=\frac{\alpha}{\sqrt{k}}\left(u_{B}-u_{D}\right) .
$$

where $u_{B}$ is the velocity at the interface, $u_{D}$ is the Darcy velocity of the fluid in the porous medium, $k$ the permeability of the material and $\alpha$ is a nondimensional parameter that represents the porous medium structure in the interface region.

Due to few physical experiments, Beavers and Joseph work of singlephase flow is still a representative physical experiment about the coupling flow problem[3]. The boundary condition proposed by them was theoretically validated by Saffman[6] for similar experiments. He proposed a more generic boundary condition, the Beavers-Joseph-Saffman interface condition presented in Eq.1-2.

$$
u_{B}=\frac{\sqrt{k}}{\alpha} \frac{d u}{d n}+O(k)
$$

where $O(k)$ is the average velocity in the porous medium and $n$ is the normal 
direction of the interface.

Mikelić and Jäger[7] further confirmed the Beavers-Joseph-Saffman boundary condition by theoretical study deducing the same interface condition based on the homogenization theory[3][8]. Despite Saffman[6], Jones[9] and, Liu and Prosperetti[10] have proposed new boundaries conditions for generic cases, BJ boundary condition is considered a special case for one-dimensional channel flows.

Beavers and Joseph[5], Beavers[11], Richardson[12], Beavers et al.[13] and Goyeau[14] pointed that the parameter $\alpha$ has a strong relation to the structure of the porous media at interface. Even materials with similar permeability may have different slip coefficients[15]. Larson and Higdon [16] concluded in their numerical study that is not possible to define a consistent $\alpha$ value for any media.

Yao and Huang[3] presented an extensive review of the coupling theory of porous media-free flow since the relevance of Beavers and Joseph work until validations and other scientific contributions to apply this knowledge in a discrete fractured-vuggy network model.

An alternative way to two-domain approach is to use Brinkman formulation. A single differential equation is used in both the macropores and porous matrix domains, without the need of an explicit interfacial boundary condition. The equations parameters are set such that Darcy's model is recovered in the porous matrix and Stokes equation is recovered in the macropores (vugs). This model establishes a seamless transition between Darcy and Stokes zones.

Brinkman[17] first studied the calculus of the viscous force on a dense swarm of particles. The use of a unified approach avoids some problems faced in coupled equations[18]. This model is currently used as a general momentum equation for a mixture of two materials, one incompressible fluid and another composed by rigid solid[15][19].

The work of Taylor [20] showed that the BJ condition can be deduced from the Brinkman equation. Neale and Nader [21] demonstrated that the predictions of Beavers-Joseph and Brinkman's [22] extension of Darcy's law are identical in seepage region.

The single-domain Brinkman model and the multiple-domain StokesDarcy model were compared by Le Bars and Worster[23] using flow through adjacent porous media and free fluid region during binary alloy solidification. Defining a viscous transition zone inside the porous domain, a good agreement between the two formulations for the solid, liquid and mushy layer was obtained.

Many papers[4][24][25][26][2][18][27] on karst reservoirs consider a 2D 
synthetic geometry composed by perfect cavities (ellipsis and circles), connected by fractures or isolated, as a representation of the rock. StokesBrinkman equation is used in many of them in numerical formulation considering single-phase steady-state flow. Simplified modeling approach facilitates the flow simulation and is less computationally expensive being an effective alternative to obtain information about the main flow characteristics.

Arbogast et al.[4] solve Darcy-Stokes coupled equations in a macro-model for vuggy porous media using the finite element method. The computational study considered synthetic vuggy media with layered vugs, meandering vug channels, constricted vug channels, and isolated vugs to accurately predict the effective flow properties in this type of aquifers on the field scale. The results showed that vug interconnectivity is the most critical variable in predicting effective permeability.

Using a mixed finite element method, Popov et al.[24] solve Brinkman's model at fine-scale to study flow through vuggy porous media with isolated and connected vugs by different types of fracture networks. The analysis showed that Stokes-Brinkman equations can be used to model physical effects pertaining to vugular porous media on a fine-scale model. Taking these equations to a coarser scale, the presence of short fractures connecting large vugs increase the effective permeability by a factor of 2-4 remaining in the same order of magnitude. Long fractures with large apertures changed the effective permeability by many orders of magnitude.

Multi-scale approaches and numerical solution of Brinkman model were used in Gulbransen et al.[25] and Qin et al. [26] on artificial carbonate karst formation composed by fractures and cavities. A multiscale mixed finite element method is applied by Gulbransen et al.[25] to solve fluid transport on a fine scale. The basis functions extracted in the first step are subsequently used in Darcy equation solution to approximate pressure and velocity on a coarse grid.

Upscaling analysis of vuggy porous media using Brinkman equation was successfully applied by Qin et al.[26]. In this paper, the effective permeability is obtained from local solution on fine scale and then the upscaled permeability is used to solve Darcy equation on coarse scale. It also shows that global scale-up gives better results than local scale-up.

In the same way as Krotkiewskiet al.[28], this study proposes to estimate permeability based on simulations made in a digital rock physics of carbonate samples. It was used the same model to calculate the equivalent permeability of carbonate karst rock samples in 3D but used Darcy or Stokes in some parts of the domain. 
Brinkman model was used by them when the ratio between crack and matrix permeability does not exceed four orders of magnitude, uses Darcy in the low permeability matrix and Stokes in large channels. This approach was employed to reduce the computational cost of the 3D model. The void space connection proved once again to be an important factor in determining the effective permeability.

In He et al.[18] research, it was also employed Brinkman model in 2D synthetic representations of naturally fractured carbonate karst reservoirs. The application of this model in a single-phase transient flow properly described fluid flow in both domains. Their results showed that the presence of cavities has a significant impact on fluid transport in this type of heterogeneous media.

Pore space representations of vuggy porous media were used in Okabe and Blunt[29], and Golfier et al.[27] to predict equivalent permeability. Okabe and Blunt[29] analyzed the permeability under the control of vugs and smallscale pores, managing to capture the behavior of computed permeability in relation to macroporosity. The multi-scale study combined microtomography and multiple-point statistics images for a Cretaceous carbonate rock and compute the permeabilities using Lattice Boltzmann method. The average of computed permeability reached a good agreement with the measured value for a small sample.

Golfier et al.[27] focused on macroscopic behavior using the DarcyBrinkman model. Representations of fractured and cavity porous media are numerically solved. An explicitly analytical solution for effective permeability is derived and compared to the numerical solution. The relation obtained of effective global permeability of a two-dimensional vuggy system made of circular fluid inclusion showed consistent values for a certain range of dimensionless porous region permeability and macroporosity, $k_{\omega} / l^{2} \lesssim 10^{-4}$ and $R \ll l$.

Previously, in our research group, a dissertation [2] on numerical simulation in two dimensions was done using Brinkman's model in simple geometries - pores and fractures, connected or not - besides preliminary studies with core samples. Now, we use this knowledge to deepen the simulation on several slices of microtomographic images of carbonate samples.

The first focus was to demonstrate the efficiency of the Brinkman model in describing the transport of fluids at the interface between free flow and porous surface. For this, we use a geometry composed by a channel and a porous material inspired in the Beavers and Joseph experiment and compare the obtained data. In sequence, micro-tomographic images of carbonate samples were used to create a geometry composed by vugs and porous matrix, to 
determinate the equivalent permeability based on flow simulation.

\section{5}

\section{Delimitation of the study}

This thesis aims to analyze the behavior of the flow in porous media with the presence of macropores. Simulations were carried out on two core samples of carbonate rocks taken from the Morro do Chaves Formation in the Sergipe-Alagoas Basin located in northeastern Brazil. These samples are considered analogous to those found in oil reservoirs on the Brazilian east coast pre-salt reservoirs. The simulations were done in two dimensions based on the tomography slices of these samples where the macropores were identified and later classified as vugs.

Several softwares were used in the characterization phase of the samples, vug geometry construction, mesh generation and flow modeling. The simulated flow is in steady state in the presence of only one fluid. We impose a pressure gradient on the $2 \mathrm{D}$ section. Numerous simulations were done by the program developed in Python with the addition of the FEniCS library where geometry, mesh size, flow direction, pressure gradient, material permeability and fluid viscosity were varied.

\section{6 \\ Division of Chapters}

\section{Chapter 1: Introduction}

Chapter $\S 1$ gives a brief introduction of the subject, defines the problem and motivation. Also, it shows the objectives and delimitation of this dissertation.

\section{Chapter 2: Literature review}

Chapter $\S 2$ presents an analysis of the information contained in the literature and previous work on flow in porous media, type of porous media, the scale of investigation and description of finite element method.

\section{Chapter 3: Methodology}

Chapter $\S 3$ presents the main equations, its fundamentals and the aspects of the numerical methodology employed to solve the flow. It also presents the variational form implemented in the computational simulation.

\section{Chapter 4: Model Analysis}


Chapter $\S 4$ starts with mesh testing and numerical validation of the model. The model developed in this work was analyzed under different conditions for the problem of flow between permeable surface and free flow. The synthetic model was constructed to be a the reproduction of the BeaversJoseph experiment. The results of this analysis was compared to the Beavers and Joseph data. After, it presents the results obtained with the simulation in two carbonate samples.

\section{Chapter 5: Results}

Chapter $\S 5$ presents the methodology used to create a $2 \mathrm{D}$ virtual representation of rocks. The simulation tool described in chapter $\S 4$ is used and some validations were presented. After, it shows the results obtained with the flow simulation in two carbonate samples.

\section{Chapter 6: Discussions and Conclusions}

Based on the results obtained by the program, we discuss the conclusions for the phenomenon investigated and suggest future works. 


\section{2}

\section{Literature Review}

The bibliographic review chapter was divided into 3 sections. First, basic concepts about porous media flow are introduced. It also includes a brief introduction to the most common average techniques to homogenizing the permeability of a non-homogeneous reservoir. The second section presents the type of rock studied and used to build virtual representations of small segments of a reservoir. The third section presents a numerical method that is crucial in this work to solve the partial differential equations of the flow problem.

\section{1}

\section{Porous Media Flow}

A permeable porous medium is a type of material that contains connected pores that allow the flow of fluids through it[30]. Porosity and permeability are important properties of a porous medium. Porosity is how much empty space exists in a material in relation to its total volume as eq. 2-1, therefore, it measures the ability to retain fluids. These voids are formed between the grains of the solid matrix and vary in size depending on the shape, packaging and selectivity of these forming particles.

$$
\phi=\frac{V_{\text {pores }}}{V_{\text {total }}}
$$

The permeability tells us how easily the fluid flow through a porous medium. Permeability is a function of the porous structure, including pore size distribution and connectivity[31].

For the hypothesis that the permeability does not have a uniform value for the system, an average value for the permeability of the system can be calculated using typical methods for permeability upscaling, that are weighted (eq.2-2a), harmonic (eq. 2-2b) and geometric (eq.2-2c). In this case, the region can be approximated to a system in layers, blocks or rings with different permeabilities[32]. These representative values of given regions, represented 
in fig. 2.1[33], are used to upscale the properties.

$$
\begin{aligned}
& k_{\text {avg }}=\frac{k_{1} \cdot h_{1}+k_{1} \cdot h_{2}+\ldots+k_{n} \cdot h_{n}}{h_{1}+h_{2}+\ldots+h_{n}}=\frac{\sum_{i=1}^{n} k_{i} \cdot h_{i}}{\sum_{i=1}^{n} h_{i}} \\
& k_{\text {avg }}=\frac{l_{1}+l_{2}+\ldots+l_{n}}{\frac{l_{1}}{k_{1}}+\frac{l_{2}}{k_{2}}+\ldots+\frac{l_{n}}{k_{n}}}=\frac{\sum_{i=1}^{n} l_{i}}{\sum_{i=1}^{n} \frac{l_{i}}{k_{i}}} \\
& k_{\text {avg }}=\sqrt[n]{k_{1} \cdot k_{2} \ldots k_{n}}
\end{aligned}
$$

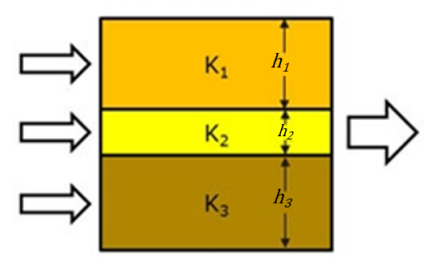

(a) Weighted-average

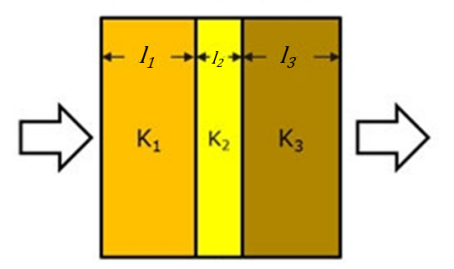

(b) Harmonic-average

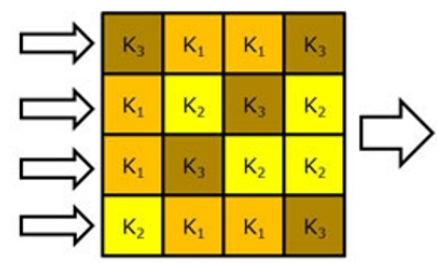

(c) Geometric-average

Figure 2.1: Permeability combinations. From Exploration and Production Geology.

For a linear flux of an incompressible fluid through parallel and horizontal beds is used weighted-average permeability. The harmonic-average is used when the flow direction is perpendicular to the beds, layers in series, and geometric when there is no apparent flow preference for any direction, in this case, the beds are similar to the blocks.

\section{2}

\section{Carbonate Rocks}

The generic term coquinas represents rocks composed by the accumulation of shells of diverse origins[34]. The variability of the depositional and diagenetic process contributes to the high heterogeneity, which makes these reservoirs complex, thus interfering with the predictability of the flow behavior.

Coquinas are sedimentary rocks consisting mainly of calcium carbonate. According to Terra[35], carbonates are rocks composed of carbonaceous particles (forming grains) - which may be peloids, oncolites, oolites, spherulites, bioclasts and intraclasts - micrite (microcrystalline calcite) and very often cement. Figure 2.2 shows the Dunham classification expanded by Embry \& 
Klovan for carbonate rocks, which is widely used and is based on depositional texture. This method classifies rocks based on the mud-grains relation.

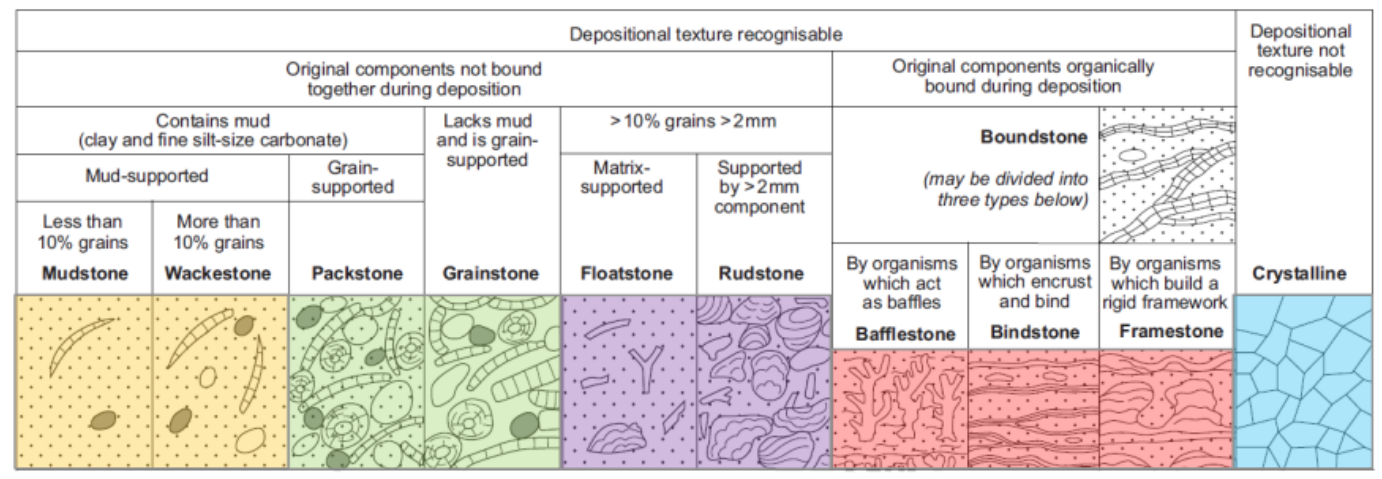

Figure 2.2: Dunham (1967) classification for carbonate sedimentary rocks with Embry \& Klovan (1971) modifications. From Nichols, 1999.

The discovery of carbonate reservoirs in the 1950s in different parts of the world stimulated research[35][36]. Later, carbonate rocks were found in some Brazilian reservoirs and, currently, most of the known reserves are found in sandstones and carbonates[1][37]. Nowadays, the study of this type of rock has become important again due to the exploration of large offshore deposits in the pre-salt range. The determination of the porosity, permeability and connectivity allows for a better understanding of the behavior of the system, refining the prediction of reserves and fluid flow[38].

We can classify carbonate porosity according to Choquete \& Pray[39], which is based on the relation between porosity and fabric, as shown in fig. 2.3. In the fabric selective group are the interparticles, intraparticles, fenestral, shelter and framework costitute the primary porosity type, which is formed during sedimentation; and intercrystalline and moldic establish the secondary porosity, developed after deposition. In the group with no relation between porosity and fabric (not fabric selective) are channel, fracture and vuggy[40]. There are also caverns, which are holes the size of a man. 

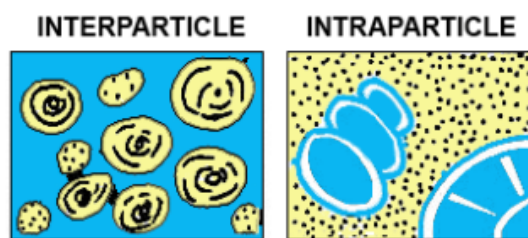

INTRAPARTICLE
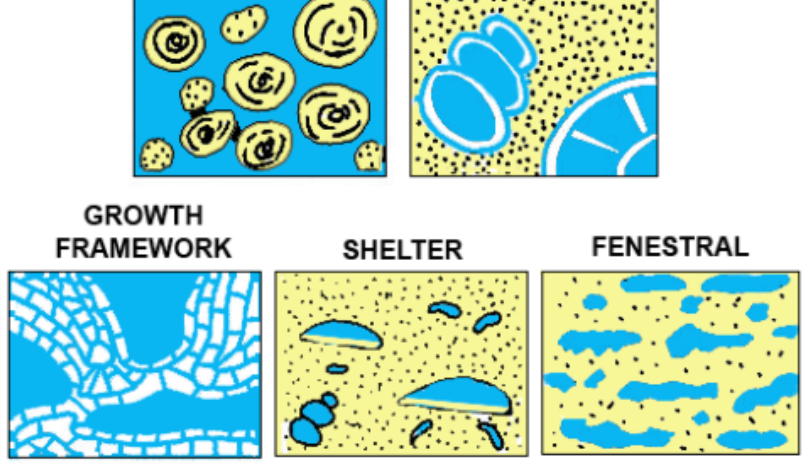

INTER-
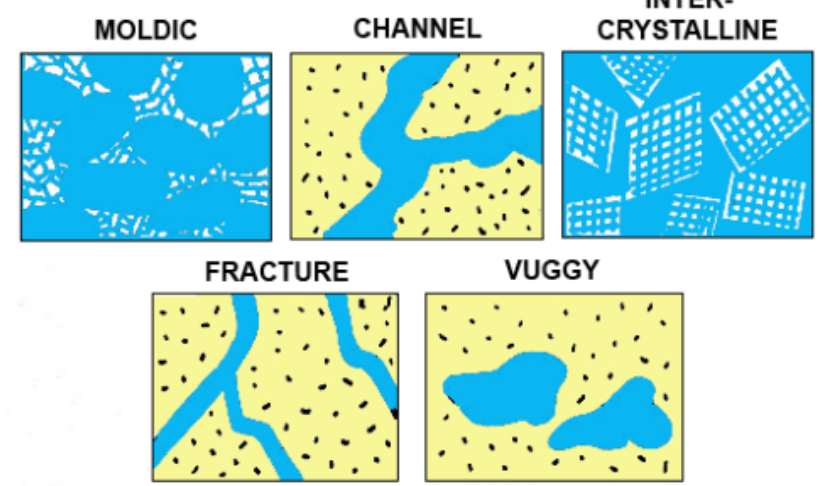

Figure 2.3: Porosity types usually found in carbonates. From Choquette \& Pray, 1970.

The difference between moldic and vuggy is the selection of the dissolution of the material, where, in the moldic, it would be only one material, which could be grain or matrix; while in the vugs, there are more than one. In our case, we will analyze the vug porosity that comes from the dissolution of the shell together with part of the matrix.

The core samples under investigation are coquinas, a type of carbonate rocks, with large vugs. The interest is to observe the flow behavior in this heterogeneous rock and how the presence of vugs changes the equivalent permeability. A methodology to describe the relation between macroporosity and equivalent permeability is the main goal.

\subsection{1}

\section{Finite element method}

The Finite Element Method (FEM) is a method for numerical solution of partial differential equations (PDE's), in which a continuous medium is subdivided into multiple small parts, elements, which are connected by nodes[41]. This is a tool used to determine solutions, mainly to those problems that do not have an exact solution. The approximate solution is a projection 
of the exact solution in a finite-dimensional subspace[42].

The method presented is widely used to solve engineering problems, such as fluid dynamics, due to its robustness and efficiency. Some typical steps used in problem-solving by finite element analysis are described in fig. 2.4.

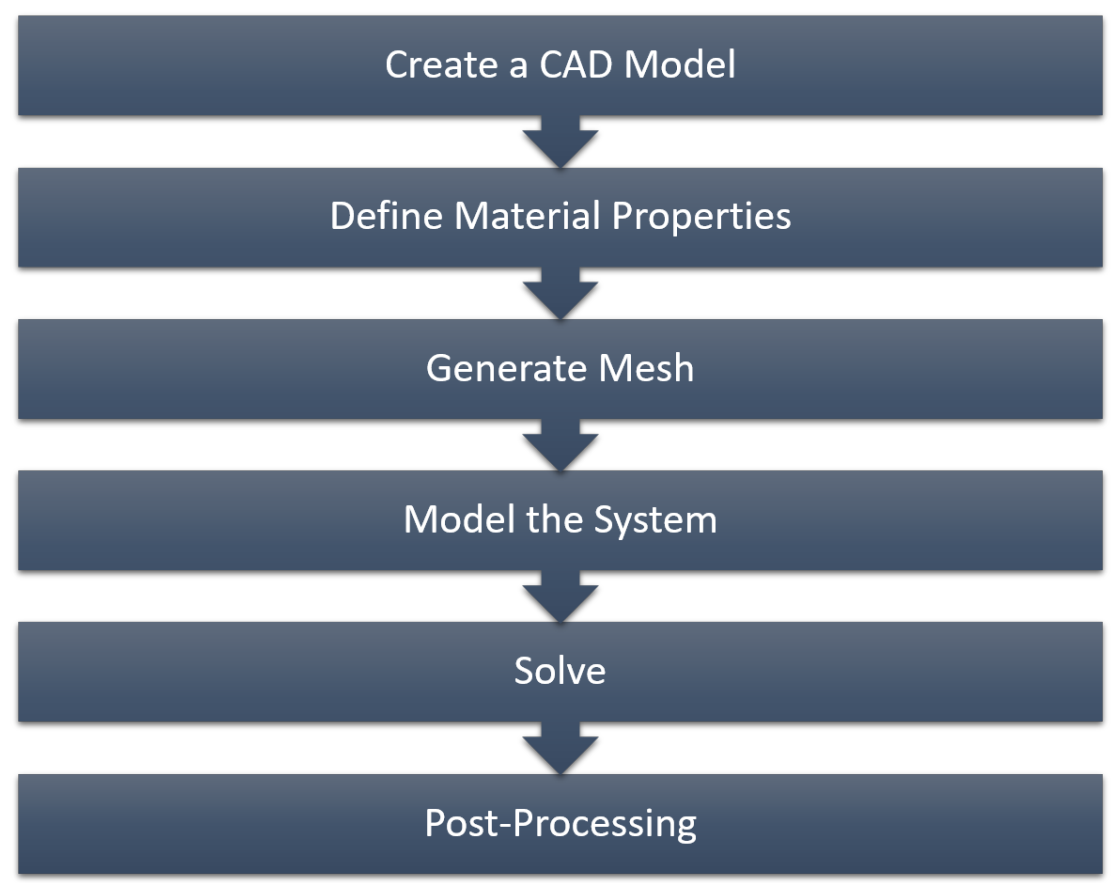

Figure 2.4: Steps of Finite Element Analysis

Firstly, a representative geometric model of the system is constructed as a CAD model. Then, a mesh is generated for the geometry, thus dividing the CAD model into numerous small pieces. From there, it is required to model the problem, the definition of the constraints, boundary conditions and parameters. In the FEM, the differential equation is transformed into a set of algebric equations. The solution of the linear system yields the approximate solution. The post-processing step is the graphic representation of the solution.

Next chapter presents the mathematical model selected and the application of the variational formulation required by the finite element method. 


\section{3}

\section{Methodology}

For this study, a mathematical model is developed based on the equations of mass and momentum conservation. The Brinkman equation is used, which combines Darcy and Stokes equations in a single partial differential equation [22], making it possible to model the fluid flow in the porous medium and in the voids with a single equation.

The finite element method (FEM) will be used to find an approximate solution for the partial differential equation (PDE) for flow through a $2 \mathrm{D}$ section. The code will be implemented in the Python programming language in conjunction with the Dolfin library, developed in the FEniCS [43] project for the PDE solution using FEM.

This chapter presents the mathematical formulation of the numerical methodology used to solve the coupled equations that describe the flow through porous media with macropores.

\section{1}

\section{Mathematical modeling}

This section presents the set of differential equations that govern the flow in vuggy porous media based on the mathematical formulation developed by Brinkman [22]. The variational form of the Brinkman equation is also derived in order to obtain a solution by the finite element method.

\subsection{1}

\section{Conservations equations}

Mass conservation is described by eq. 3-1.

$$
\frac{\partial \rho}{\partial t}+\nabla \cdot(\rho \mathbf{u})=0
$$

where $\rho$ is the liquid density, and $\mathbf{u}$ is the velocity field.

The specific mass of the an incompressible fluid is independent of the spatial coordinates and time. So, the continuity equation reduces to: 


$$
\nabla \cdot \mathbf{u}=0
$$

\subsection{2}

\section{Conservation of linear momentum}

The conservation of momentum for incompressible fluids is described by the Cauchy equation. For a Newtonian fluid, it becomes the Navier-Stokes equation.

\subsubsection{1}

\section{Navier-Stokes Equations}

Navier-Stokes equation (3-3) and continuity equation (3-2), when coupled, form a set of nonlinear second order partial differential equations that can not be solved analytically in most situations, except for basic cases where the initial conditions, boundary conditions and geometries are simple[44].

$$
\rho\left(\frac{\partial \boldsymbol{u}}{\partial t}+\boldsymbol{u} \nabla \cdot \boldsymbol{u}\right)=\rho \boldsymbol{g}-\nabla p+\mu \nabla^{2} \boldsymbol{u}
$$

For slow velocity flows at which inertia is negligible, i.e., for low Reynolds number flows, the Navier-Stokes equation is simplified to Stokes equation:

$$
\rho \boldsymbol{g}+\mu \nabla^{2} \boldsymbol{u}=\nabla p
$$

\subsubsection{2}

\section{Darcy's Law}

Proposed by french engineer Henry Darcy during experiments on water filtration in sand beds in 1856, Darcy's Law describes flow behavior through a permeable material.

$$
Q=-\frac{k}{\mu} A \nabla p
$$

The flow rate per unit area is proportional to the pressure gradient and inversily proportional to the fluid viscosity. The proportionality constant is the medium permeability. 


\subsubsection{3}

\section{Brinkman Equation}

The Brinkman equation (3-6) is an extension of Darcy's law to describe both the flow through a porous material and in an open channel. Therefore, Brinkman suggested a general interpolation equation that combines Darcy's law, which governs slow flow in porous media, and Stokes equation, in order to describe flow in a channel[22][17].

$$
\nabla p=-\mu \boldsymbol{k}^{-1} \boldsymbol{u}+\mu^{*} \nabla^{2} \boldsymbol{u}
$$

where $p$ is the pressure, $\mu$ the fluid viscosity, $\mu^{*}$ a model parameter, $\boldsymbol{u}$ the velocity field and $\boldsymbol{k}$ the permeability tensor of the porous medium.

As sketched in fig. 3.1, when the fluid particle is inside a pore or vug, we can assume that $k$ is infinite and $\mu^{*}=\mu$, consequently, Darcy's term $\mu \boldsymbol{k}^{-\mathbf{1}} \boldsymbol{u}$ vanishes, which leads to Stokes equation. Whereas, if the particle is flowing within the porous media, we set $\mu^{*}=0$ and Darcy equation is recovered. With a choice of parameters, we change the equation term to be solved in each domain.

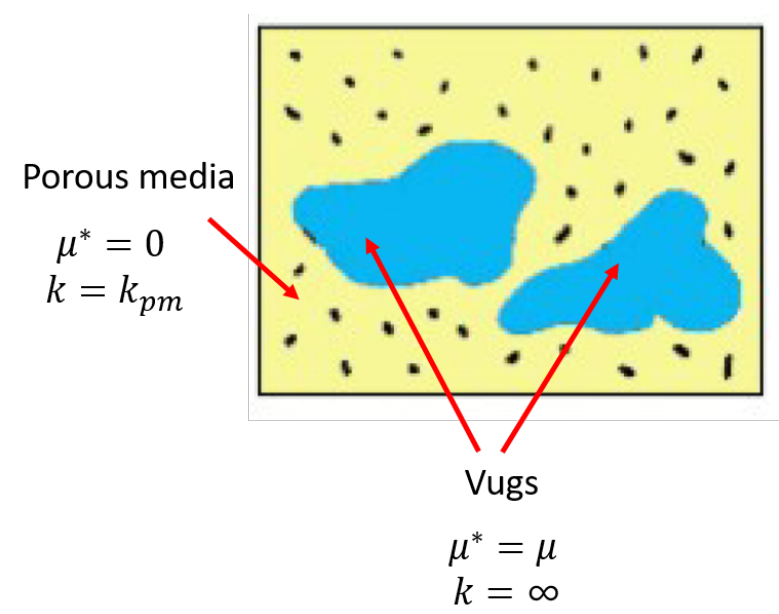

Figure 3.1: Brinkman's model. Adapted from Choquette \& Pray, 1970.

The hypothesis considered in this model to simplify the problem are steady-state regime, fully-developed laminar flow in the inlet and outlet planes, rigid porous matrix, Newtonian and incompressible liquid, isothermal flow. There is also no chemical interaction, and it was neglected gravitational and inertial effects. 


\section{2}

\section{Numerical formulation}

For the solution of the mathematical model, composed of a system of partial differential equations, the numerical approach known as finite element method is used. In this way, it is possible to obtain an approximate solution for the flow in different arrangements using the FEniCS package, a library that can be used with the python interface for solving partial differential equations.

\subsection{1}

\section{FEniCS}

FEniCS is an open-source platform for solving partial differential equations (PDEs) by finite element method. Dolfin is a library that acts as FEniCS computational backend which can be used in Python or $\mathrm{C}++$ interfaces[43].

To use this library, first, we have to transform the PDE problem into the proper weak formulation readable for FEniCS. For this, the problem has to be expressed as a variational formulation while finite elements are used for discretization in space[43].

In order to discretize the space, we have to choose the appropriate set of basis functions. Returning to eq. 3-6, we can see that the dependent variables are pressure $p$ and velocity field $\boldsymbol{u}$. Fluid viscosity $\mu$ and permeability of the porous medium $k$ are parameters of the problem.

We choose the triangular elements on fig. 3.2. This type of element belongs to the Lagrange family, also known as Continuous Galerkin, and are based on polynomial equations[45]. The element with 6 degrees of freedom on fig. 3.2b was used for velocity and the element with 3 degrees of freedom (fig. $3.2 \mathrm{a})$ for pressure. Thus, the pressure varies linearly (P1) and the velocity is based on quadratic polynomials (P2). 

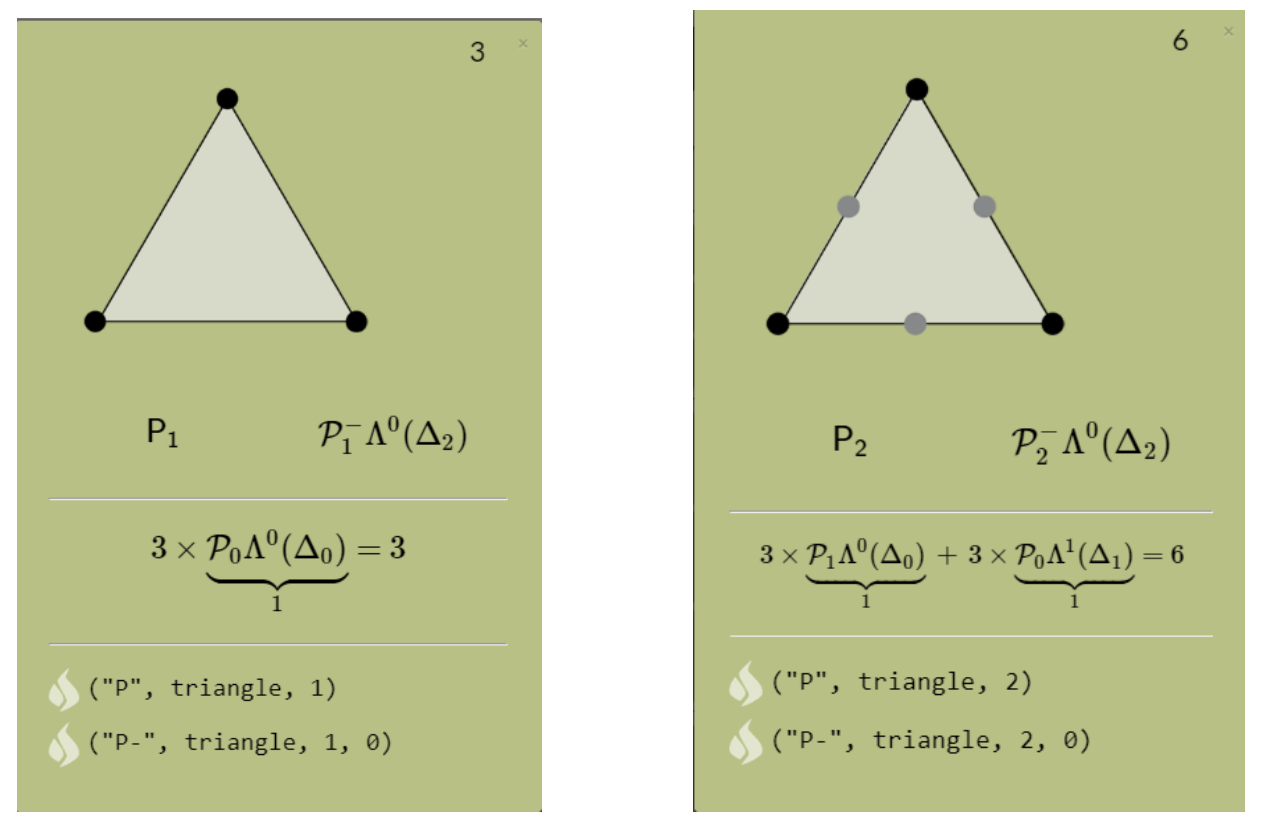

(a) edge element of 3 degrees of freedom (b) edge element of 6 degrees of freedom

Figure 3.2: Type of finite element used. From Arnold and Logg, 2014.

In the following section, the variational formulation used in this project is described.

\subsection{2}

\section{Brinkman's variational formulation}

Consider the following PDE system, the Brinkman's flow equation 3-6 presented in section \$3.1.2.3 and the continuity equation 3-2 for an incompressible fluid:

$$
\begin{aligned}
-\mu^{*} \nabla^{2} \mathbf{u}+\nabla p+\mu \mathbf{k}^{-1} \mathbf{u} & =\mathbf{f} \text { in } \Omega, \\
\nabla \cdot \mathbf{u} & =0 \text { in } \Omega .
\end{aligned}
$$

where $\mathbf{f}$ is a known function, an external force applied to the fluid and subject to the following boundary conditions:

$$
\begin{array}{cl}
\mathbf{u} \cdot \mathbf{n}=u_{0} & \text { on } \Gamma_{D}, \\
p=p_{\text {in }} & \text { on } \Gamma_{p_{\text {in }}}, \\
p=p_{\text {out }} & \text { on } \Gamma_{p_{\text {out }}} .
\end{array}
$$

where $\Gamma$ represents the domain boundary defined for the pressure boundary conditions, with $p_{\text {in }}$, the input pressure, and $p_{\text {out }}$, the output pressure, are imposed, $u_{0}$ the velocity pointing out of the boundary $\Gamma_{D}$ and $\boldsymbol{n}$ the vector 
normal to the border $\Gamma_{D}$.

The basic recipe for turning a PDE into a variational problem is take the equation as eq. 3-7a, multiply by a function $\mathbf{v}$, called weight function, and integrate over the domain $\Omega[43]$, resulting in:

$$
-\int_{\Omega} \mu^{*} \nabla^{2} \mathbf{u} \cdot \mathbf{v} \partial \Omega+\int_{\Omega} \nabla p \cdot \mathbf{v} \partial \Omega+\int_{\Omega} \mu \mathbf{k}^{-1} \mathbf{u} \cdot \mathbf{v} \partial \Omega=\int_{\Omega} \mathbf{f} \cdot \mathbf{v} \partial \Omega
$$

For the mass conservation 3-7b, we multiply this by a scalar weight function $q$ and integrate over $\Omega$ transforming into a variational form:

$$
\int_{\Omega} \nabla \cdot \mathbf{u} \cdot q \partial \Omega=0
$$

Integration by parts of terms with second-order derivatives is required[43] in order to be able to use basis functions, which have discontinuous derivatives. Following that, it will apply these concepts at the first integration on eq. 3-9.

$$
-\int_{\Omega} \mu^{*} \nabla^{2} \mathbf{u} \cdot \mathbf{v} \partial \Omega=\int_{\Omega} \mu^{*} \nabla \mathbf{u} \cdot \nabla \mathbf{v} \partial \Omega-\int_{\Omega} \mu^{*} \nabla \cdot(\mathbf{v} \cdot \nabla \mathbf{u}) \partial \Omega
$$

Using the divergence theorem, which states $\int_{\Omega} \nabla \cdot \mathbf{f} \partial \Omega=\int_{\Gamma} \mathbf{n} \cdot \mathbf{f} \partial S$, the second derivative term in the weak formulation becomes:

$$
-\int_{\Omega} \mu^{*} \nabla^{2} \mathbf{u} \cdot \mathbf{v} \partial \Omega=\int_{\Omega} \mu^{*} \nabla \mathbf{u} \cdot \nabla \mathbf{v} \partial \Omega-\int_{\Gamma} \mu^{*} \mathbf{n} \cdot(\mathbf{v} \cdot \nabla \mathbf{u}) \partial S
$$

As $\nabla u$ at the entrance is zero, eq. 3-12 becomes:

$$
\int_{\Omega} \mu^{*} \nabla \mathbf{u} \cdot \nabla \mathbf{v} \partial \Omega
$$

Moving to the second integration on eq. 3-9, the term is rewritten with integration by parts on 3-14a and the Gauss-Green's theorem on 3-14b:

$$
\begin{aligned}
\int_{\Omega} \nabla p \cdot \mathbf{v} \partial \Omega & =\int_{\Omega} \nabla \cdot(p \cdot \mathbf{v}) \partial \Omega-\int_{\Omega} p \cdot(\nabla \cdot \mathbf{v}) \partial \Omega \\
& =\int_{\Gamma} \mathbf{n} \cdot(p \cdot \mathbf{v}) \partial S-\int_{\Omega} p \cdot(\nabla \cdot \mathbf{v}) \partial \Omega
\end{aligned}
$$

Assuming an isotropic medium, the permeability $k$ becomes a scalar, thus, the variational form of eq. 3-7a becomes:

$$
\begin{array}{r}
\int_{\Omega} \mu^{*} \nabla \mathbf{u} \cdot \nabla \mathbf{v} \partial \Omega+\int_{\Gamma} \mathbf{n} \cdot(p \cdot \mathbf{v}) \partial S-\int_{\Omega} p \cdot(\nabla \cdot \mathbf{v}) \partial \Omega \\
+\int_{\Omega} \mu k^{-1} \mathbf{u} \cdot \mathbf{v} \partial \Omega=\int_{\Omega} \mathbf{f} \cdot \mathbf{v} \partial \Omega
\end{array}
$$


The canonical notation for variational problems is:

$$
a_{B}(\mathbf{u}, \mathbf{v})+b(\mathbf{v}, p)+b(\mathbf{u}, q)=L(\mathbf{v})
$$

where:

$$
\begin{aligned}
a_{B}(\mathbf{u}, \mathbf{v}) & =\int_{\Omega} \mu^{*} \nabla \mathbf{u} \cdot \nabla \mathbf{v} \partial \Omega+\int_{\Omega} \mu k^{-1} \mathbf{u} \cdot \mathbf{v} \partial \Omega \\
b(\mathbf{v}, p) & =-\int_{\Omega} p \cdot(\nabla \cdot \mathbf{v}) \partial \Omega \\
b(\mathbf{u}, q) & =-\int_{\Omega} \nabla \cdot \mathbf{u} \cdot q \partial \Omega \\
L(\mathbf{v}) & =\int_{\Omega} \mathbf{f} \cdot \mathbf{v} \partial \Omega-\int_{\Gamma} \mathbf{n} \cdot(p \cdot \mathbf{v}) \partial S
\end{aligned}
$$

The variational formulation was implemented into our code using the mathematical operators of Dolfin library. Each integral is applied to one part of the domain. The first integral which is based on the Stokes flow is used only in the vug domain. The integral associated with the Darcy equation, the second, is used only within the porous matrix domain. The pressure assumes a certain value $p_{\text {in }}$ at inlet and value $p_{\text {out }}$ is imposed on the outlet wall.

Appendix A.1 contains the implementation of the mathematical model that can be used to solve the pressure and velocity fields in a porous medium composed of two domains. The code was applied in both problems that we simulated, a channel with a bounding porous media and porous media with large vugs.

In next chapter, the model developed in this section will be applied to the problem of a flow through a channel with a permeable porous wall to study the flow behavior at the interface and if the Brinkman model can describe the behavior near matrix-vug observed in physical experiments. 


\section{4}

\section{Model analysis}

This chapter presents the analysis of the flow behavior at interface between void and porous surface. The numerical methodology to solve the set of partial differential equation composed by momentum conservation and mass is applied in a coupling flow problem. The model presented in the previous chapter was implemented as described before and the implementation was validated by obtaining solution of simple problems, such as flow between two parallel plates.

The geometry used is composed by a channel and a porous material. The results for this case was compared with the classical experimental analysis of Beavers and Joseph. An extensive analysis of the model was made in order to check the accuracy of the methodology used to describe the phenomenon observed in experimental data. The results show that Brinkman model is able to describe flow behavior at laminar channel flow over a porous surface.

\section{1}

Flow behavior near a porous solid and channel interface

We first study the flow through a channel composed of a permeable solid region and an open region, as sketched in fig. 4.1.

Since the finite element method was chosen as the solution method, one of the crucial steps is to generate a mesh based on the geometry of the problem to be studied. After create the geometry in Gmsh software, its necessary to divide it in many small parts. Triangle elements were chosen to form together a mesh. As can be seen in fig. 4.1, it was created a non-structured mesh in a simple rectangle geometry with $2 \mathrm{~cm}$ x $20 \mathrm{~cm}$. This section was divided into two sub-domains, Darcy and Stokes domain, corresponding to the porous matrix and void, respectively. This mesh is used in the simulation tool where it was imposed a horizontal flow, a pressure gradient at inlet and outlet plane, and a scalar permeability is defined for the porous section. 


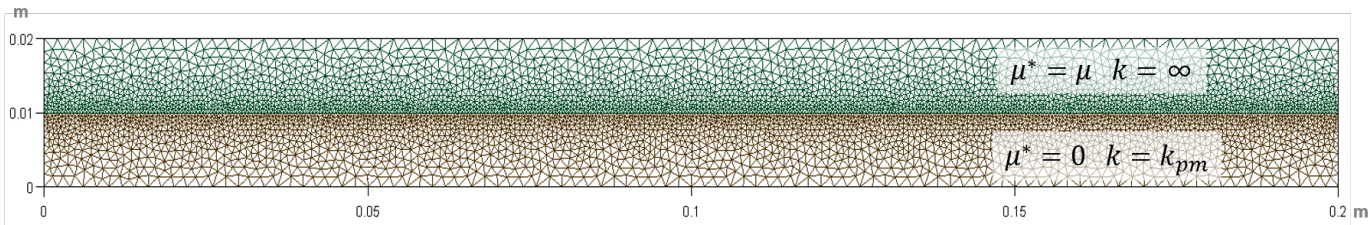

Figure 4.1: First mesh generated to be tested, where the green area is the free flow section and the brown one is the porous medium.

\subsection{1}

\section{Mesh test}

To ensure that the solution is independent of the mesh size, a mesh test was performed for the geometry shown in fig. 4.1. The flow rate at a given set of parameters was obtained for different meshes. The benchmark chosen was the values calculated for flow rate through a rectangular section. This form is composed of two regions that divide the vertical section in the middle, which a channel with a thickness of $1 \mathrm{~cm}$ is simulated, where a free flow occurs and a porous medium with $1 \mathrm{~cm}$ of height.

The hypotheses considered in the simulation are steady-state regime, fully-developed flow in the inlet and outlet planes and no-slip condition in the upper impermeable wall.

Three different meshes sizes were tested for different set of parameters: imposed pressure difference and permeability of the permeable region.

\begin{tabular}{|c|c|c|c|c|c|c|c|c|c|c|c|c|}
\hline \multicolumn{2}{|c|}{ Mesh size: } & \multicolumn{3}{|c|}{0,001} & \multicolumn{4}{|c|}{0,0005} & \multicolumn{4}{|c|}{$0,0005-0,0003$} \\
\hline & & \multicolumn{3}{|c|}{1} & \multicolumn{4}{|c|}{2} & \multicolumn{4}{|c|}{3} \\
\hline$K_{p m}\left(m^{2}\right)$ & $\Delta \mathrm{P}(\mathrm{Pa})$ & $Q_{T}\left(m^{3} / s\right)$ & $a_{h}$ & $\mathrm{Q}_{\mathrm{pm}}$ & $Q_{T}$ & $Q_{h}$ & $Q_{p m}$ & $Q_{T} \operatorname{Dev}(\%)$ & $Q_{T}$ & $a_{h}$ & $Q_{p m}$ & $Q_{T} \operatorname{Dev}(\%)$ \\
\hline \multirow[t]{5}{*}{$1,00 \mathrm{E}-20$} & 6.895 & 2,84 & 2,84 & $2,09 \mathrm{E}-14$ & 2,86 & 2,86 & $2,20 \mathrm{E}-14$ & $-0,76$ & 2,86 & 2,86 & $2,81 \mathrm{E}-14$ & $-0,09$ \\
\hline & 34.474 & 14,19 & 14,19 & $1,04 \mathrm{E}-13$ & 14,30 & 14,30 & $1,10 \mathrm{E}-13$ & & 14,31 & 14,31 & $1,40 \mathrm{E}-13$ & \\
\hline & 68.948 & 28,38 & 28,38 & $2,09 \mathrm{E}-13$ & 28,60 & 28,60 & $2,20 \mathrm{E}-13$ & & 28,63 & 28,63 & $2,81 \mathrm{E}-13$ & \\
\hline & 172.369 & 70,96 & 70,96 & $5,22 \mathrm{E}-13$ & 71,50 & 71,50 & $5,49 E-13$ & & 71,56 & 71,56 & $7,01 \mathrm{E}-13$ & \\
\hline & 344.738 & 141,92 & 141,92 & $1,04 \mathrm{E}-12$ & 143,00 & 143,00 & $1,10 \mathrm{E}-12$ & & 143,13 & 143,13 & $1,40 \mathrm{E}-12$ & \\
\hline \multirow[t]{5}{*}{$1,00 \mathrm{E}-12$} & 6.895 & 2,84 & 2,84 & $2,09 \mathrm{E}-06$ & 2,86 & 2,86 & $2,20 \mathrm{E}-06$ & $-0,76$ & 2,86 & 2,86 & $2,81 \mathrm{E}-06$ & $-0,09$ \\
\hline & 34.474 & 14,19 & 14,19 & $1,04 \mathrm{E}-05$ & 14,30 & 14,30 & $1,10 \mathrm{E}-05$ & & 14,31 & 14,31 & $1,40 E-05$ & \\
\hline & 68.948 & 28,38 & 28,38 & $2,09 \mathrm{E}-05$ & 28,60 & 28,60 & $2,20 \mathrm{E}-05$ & & 28,63 & 28,63 & $2,81 \mathrm{E}-05$ & \\
\hline & 172.369 & 70,96 & 70,96 & $5,22 \mathrm{E}-05$ & 71,50 & 71,50 & $5,49 \mathrm{E}-05$ & & 71,56 & 71,56 & $7,02 E-05$ & \\
\hline & 344.738 & 141,92 & 141,92 & $1,04 \mathrm{E}-04$ & 143,00 & 143,00 & $1,10 \mathrm{E}-04$ & & 143,13 & 143,13 & $1,40 \mathrm{E}-04$ & \\
\hline \multirow[t]{5}{*}{$1,00 E-10$} & 6.895 & 2,84 & 2,84 & $2,09 \mathrm{E}-04$ & 2,86 & 2,86 & $2,22 \mathrm{E}-04$ & $-0,79$ & 2,87 & 2,87 & $2,84 \mathrm{E}-04$ & $-0,13$ \\
\hline & 34.474 & 14,20 & 14,20 & $1,05 \mathrm{E}-03$ & 14,31 & 14,31 & $1,11 \mathrm{E}-03$ & & 14,33 & 14,33 & $1,42 \mathrm{E}-03$ & \\
\hline & 68.948 & 28,39 & 28,39 & $2,09 \mathrm{E}-03$ & 28,62 & 28,62 & $2,22 \mathrm{E}-03$ & & 28,66 & 28,65 & $2,84 \mathrm{E}-03$ & \\
\hline & 172.369 & 70,99 & 70,98 & $5,23 \mathrm{E}-03$ & 71,55 & 71,54 & $5,55 \mathrm{E}-03$ & & 71,64 & 71,63 & $7,11 \mathrm{E}-03$ & \\
\hline & 344.738 & 141,97 & 141,96 & $1,05 \mathrm{E}-02$ & 143,09 & 143,08 & $1,11 \mathrm{E}-02$ & & 143,28 & 143,27 & $1,42 \mathrm{E}-02$ & \\
\hline \multirow[t]{5}{*}{$1,00 \mathrm{E}-09$} & 6.895 & 2,85 & 2,85 & $2,13 \mathrm{E}-03$ & 2,88 & 2,88 & $2,35 \mathrm{E}-03$ & $-1,09$ & 2,89 & 2,89 & $2,93 \mathrm{E}-03$ & $-0,45$ \\
\hline & 34.474 & 14,24 & 14,23 & $1,06 \mathrm{E}-02$ & 14,40 & 14,39 & $1,17 \mathrm{E}-02$ & & 14,46 & 14,45 & $1,46 \mathrm{E}-02$ & \\
\hline & 68.948 & 28,49 & 28,46 & $2,13 \mathrm{E}-02$ & 28,80 & 28,77 & $2,35 \mathrm{E}-02$ & & 28,93 & 28,90 & 2,93E-02 & \\
\hline & 172.369 & 71,21 & 71,16 & $5,31 \mathrm{E}-02$ & 71,99 & 71,93 & $5,87 \mathrm{E}-02$ & & 72,32 & 72,24 & $7,32 \mathrm{E}-02$ & \\
\hline & 344.738 & 142,43 & 142,32 & $1,06 \mathrm{E}-01$ & 143,98 & 143,86 & $1,17 \mathrm{E}-01$ & & 144,63 & 144,48 & $1,46 \mathrm{E}-01$ & \\
\hline \multirow[t]{5}{*}{$1,00 \mathrm{E}-08$} & 6.895 & 2,94 & 2,92 & $2,28 \mathrm{E}-02$ & 3,05 & 3,02 & $2,64 \mathrm{E}-02$ & $-3,82$ & 3,15 & 3,12 & $2,96 \mathrm{E}-02$ & $-3,28$ \\
\hline & 34.474 & 14,69 & 14,58 & $1,14 \mathrm{E}-01$ & 15,26 & 15,12 & $1,32 \mathrm{E}-01$ & & 15,76 & 15,61 & $1,48 \mathrm{E}-01$ & \\
\hline & 68.948 & 29,39 & 29,16 & $2,28 \mathrm{E}-01$ & 30,51 & 30,25 & $2,64 \mathrm{E}-01$ & & 31,51 & 31,22 & $2,96 \mathrm{E}-01$ & \\
\hline & 172.369 & 73,47 & 72,90 & $5,71 \mathrm{E}-01$ & 76,28 & 75,62 & $6,61 \mathrm{E}-01$ & & 78,79 & 78,04 & $7,41 \mathrm{E}-01$ & \\
\hline & 344.738 & 146,95 & 145,81 & $1,14 \mathrm{E}+00$ & 152,56 & 151,24 & $1,32 E+00$ & & 157,57 & 156,09 & $1,48 \mathrm{E}+00$ & \\
\hline
\end{tabular}

Table 4.1: Comparison of results for mesh refining. 
The results for each mesh are shown in tab. 4.1. Five values of permeability of the porous matrix was defined for the porous domain and five values of pressure gradient at inlet and outlet planes. The choice of the best mesh was guided by the total flow rate. The flow rate was calculate integrating the velocity at each node of the outlet wall. The third mesh tested was chosen because it had little variation of the result of the simulation in relation to the others and a greater amount of elements in the border between the two regions. For this mesh, the values for the flow differed by a maximum of $3.28 \%$ in the case of a porous medium with high permeability. The element size varies between $3 \times 10^{-4}$ and $5 \times 10^{-4}$, where the smallest element size was used to increase the accuracy of the calculations near the interface between the two regions and the largest at the top and bottom wall of the domain.

In order to validate our numerical implementation, the permeability of the porous wall was set to a value close to zero, such that all the flow occurred through the void. The flow rate was compared to the analytical solution of the flow through parallel plates. The results are shown in tab. 4.2. The deviation values are the same because the problem solved is linear.

\begin{tabular}{|r|cc|c|}
\cline { 2 - 3 } \multicolumn{1}{c|}{} & \multicolumn{2}{c|}{ Flow rate $\left(\mathrm{m}^{\mathbf{3}} / \mathrm{s}\right)$} & \multicolumn{1}{c}{} \\
\hline \multicolumn{1}{c|}{$\Delta \mathbf{P}(\mathbf{P a})$} & Poiseuille & Simulation & Deviation \\
\hline $6.894,76$ & 2,87 & 2,86 & $0,16 \%$ \\
$34.473,80$ & 14,34 & 14,31 & $0,16 \%$ \\
$68.947,60$ & 28,67 & 28,63 & $0,16 \%$ \\
$172.369,00$ & 71,68 & 71,56 & $0,16 \%$ \\
$344.738,00$ & 143,35 & 143,13 & $0,16 \%$ \\
\hline
\end{tabular}

Table 4.2: Comparison of the values obtained between Poiseuille's flow and this numerical model. 


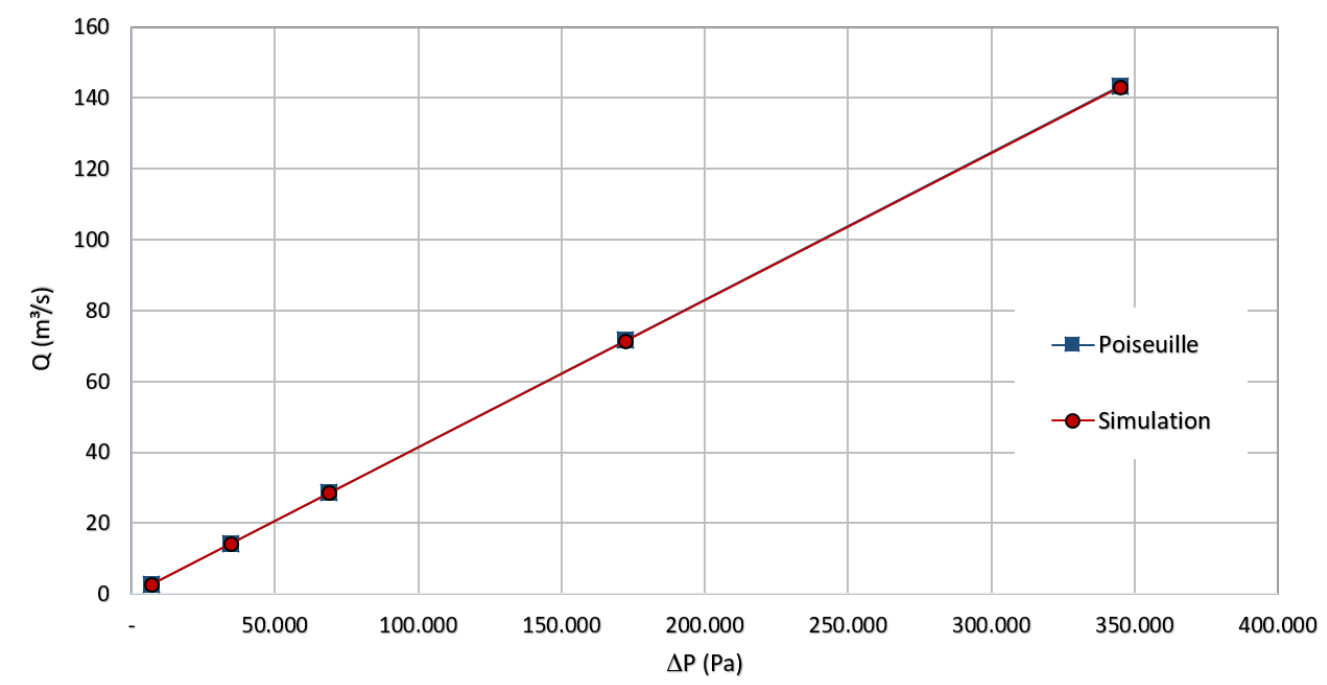

Figure 4.2: Comparison between Poiseuille and our numeric model.

The numerical solution deviated only by $0.16 \%$ from the analytical Poiseuille equation, Eq.4-1. The same deviation is expected for the same imposed conditions because the problem is linear.

$$
Q=-\frac{d p}{d x} \frac{h^{3}}{12 \mu}
$$

The results for 5 pressure values imposed and permeability of the lower region defined as $k_{p m}=1 \times 10^{-20} \mathrm{~m}^{2}$ also reproduced the Poiseuille solution, as shown in fig. 4.2. The code was validated by this analysis.

\section{2}

\section{Flow behavior at solid/void interface}

Beavers and Joseph (BJ) [5] proposed a semi-empirical boundary condition for an interface between a channel and a permeable porous medium. This experiment in a channel formed by an impermeable wall on top of a permeable surface provides a velocity profile in the channel similar to Poiseuille flow. However, as a consequence of the permeable surface presence, the velocity at the interface is not zero, showing an apparent slip velocity.

It was observed that there was a viscous shear effect on the permeable material in a boundary layer region[21], producing a velocity distribution shown in fig. 4.3. Based on the slip velocity at the interface between the channel and the porous medium, they proposed a boundary condition for this transition zone, in order to enable the calculation of this type of problem through the coupling between Darcy's law and Stokes equation. 


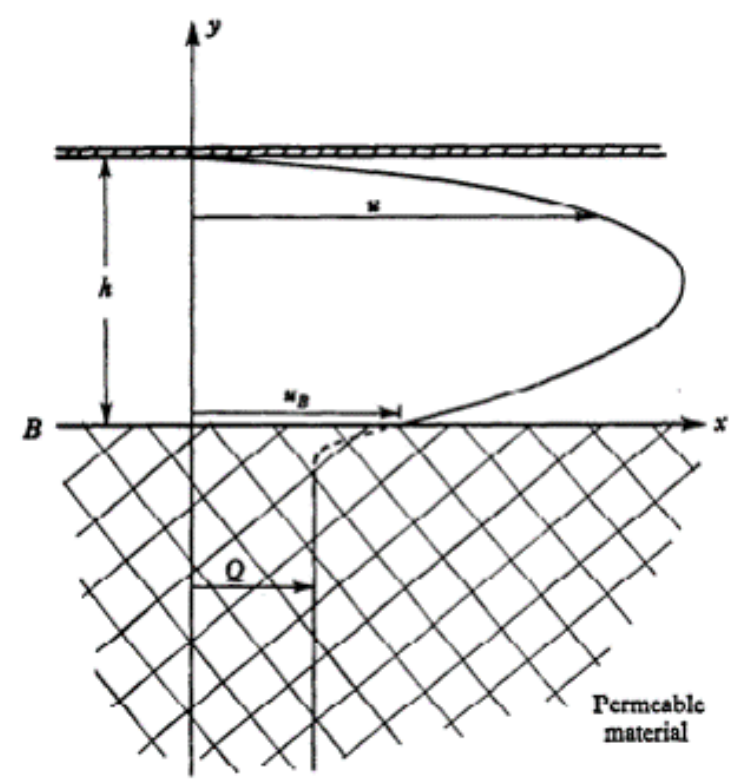

Figure 4.3: Speed profile proposed by Beavers and Joseph. From Beavers \& Joseph, 1967

In order to investigate the flow prediction of the Brinkman model, used in this work, near the surface, a parametric analysis is performed to compare the results with the Beavers and Joseph experiments. In these computations, a pressure gradient was imposed through the inlet and outlet of both domains.

Figure 4.4 shows an almost parabolic profile in the void region and a very low velocity in the permeable solid matrix of the porous medium. Figure 4.5 presents detail of the velocity field near the interface between the open channel and permeable region. As expected, the slip velocity increases as the permeability of the porous region rises.

Figure 4.6 shows the velocity field for the same mesh with different permeability values of the porous medium and $\Delta P=6,894.76 \mathrm{~Pa}$. The velocity values within the permeable material are close to zero for the three cases if compared with the free flow at the channel. A parabolic profile is formed in the channel region. Figure 4.6a presents a similar profile reached by physical experiments near the interface between domains, $\mathrm{h}=0.01 \mathrm{~m}$. With the velocity profile observed in Figs. 4.5 and $4.6 \mathrm{a}$, it is possible to confirm that the Brinkman model is able to reproduce the same velocity profile reported in experiments without the necessity of a formulation for the boundary.

To verify the accuracy of the predicted velocity field, the results were compared to the experimental values presented by Yao and Huang[3] which are also based on the BJ experiment. The same properties established in their experiment were used, where the pressure gradient is $-0.33 \mathrm{~Pa} / \mathrm{m}$ and the fluid used is water with its viscosity being 0.001 Pa.s. Another mesh is constructed. 
The representative porous media in this model is similar to that of figure 4.1, having a permeability of $k_{p m}=5 \times 10^{-9} \mathrm{~m}^{2}$ and a rectangular geometry of 20 $\mathrm{cm}$ by $5 \mathrm{~cm}$. The height of the free flow region is $2 \mathrm{~cm}$ and $5 \mathrm{~cm}$ for the porous medium. The mesh composed by the sum of the described porous medium and the channel has $20 \mathrm{~cm}$ of extension and $7 \mathrm{~cm}$ of height.

Figure 4.7 show that the velocity profile predicted here matches well the one presented by Yao and Huang, with similar slip velocity.

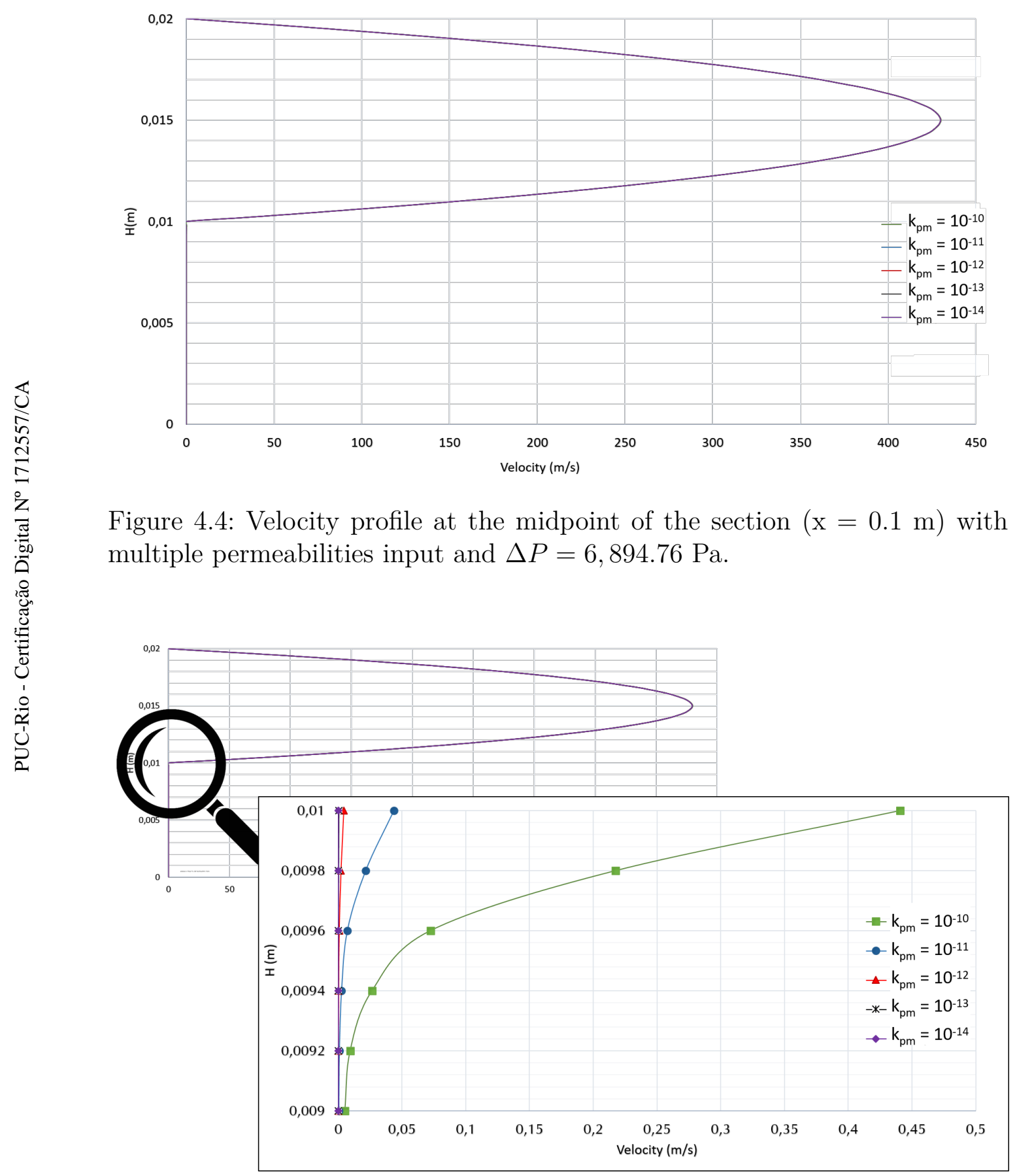

Figure 4.5: Zoom in at the interface $(\mathrm{y}=0.01 \mathrm{~m})$ between free section and permeable wall to show the slip velocity above the interface. 

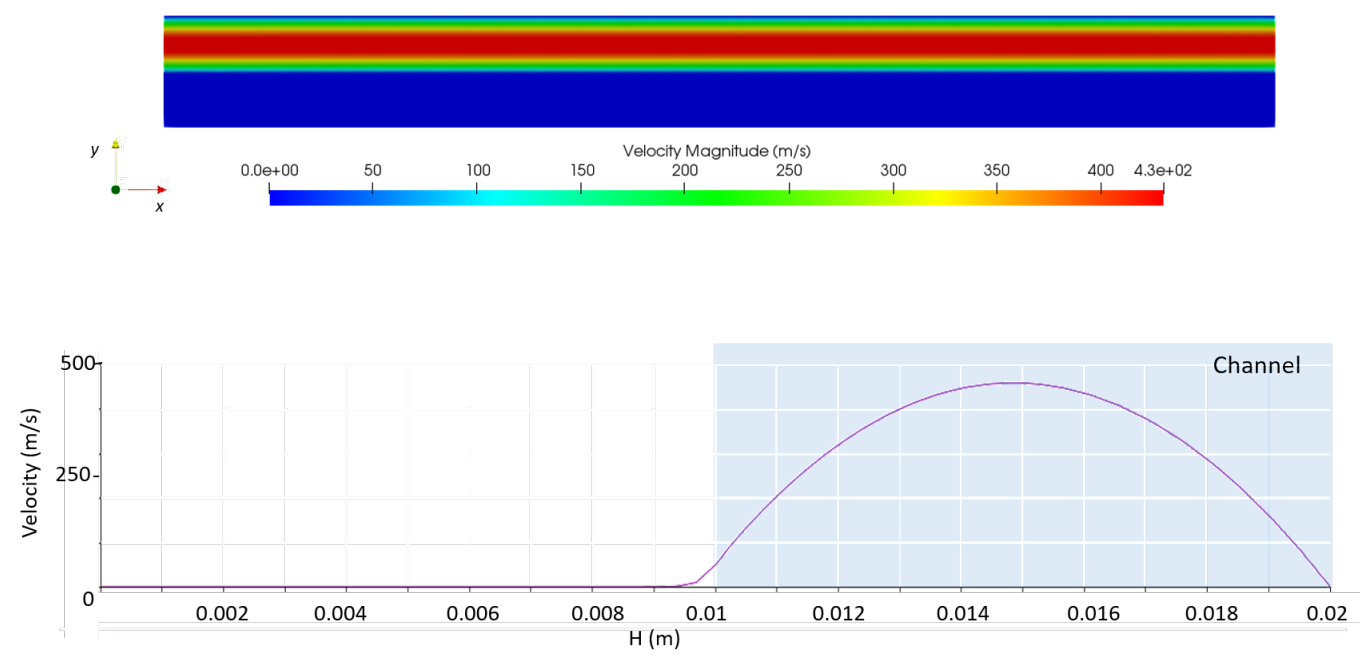

(a) $k_{p m}=1 \times 10^{-8} m^{2}$.
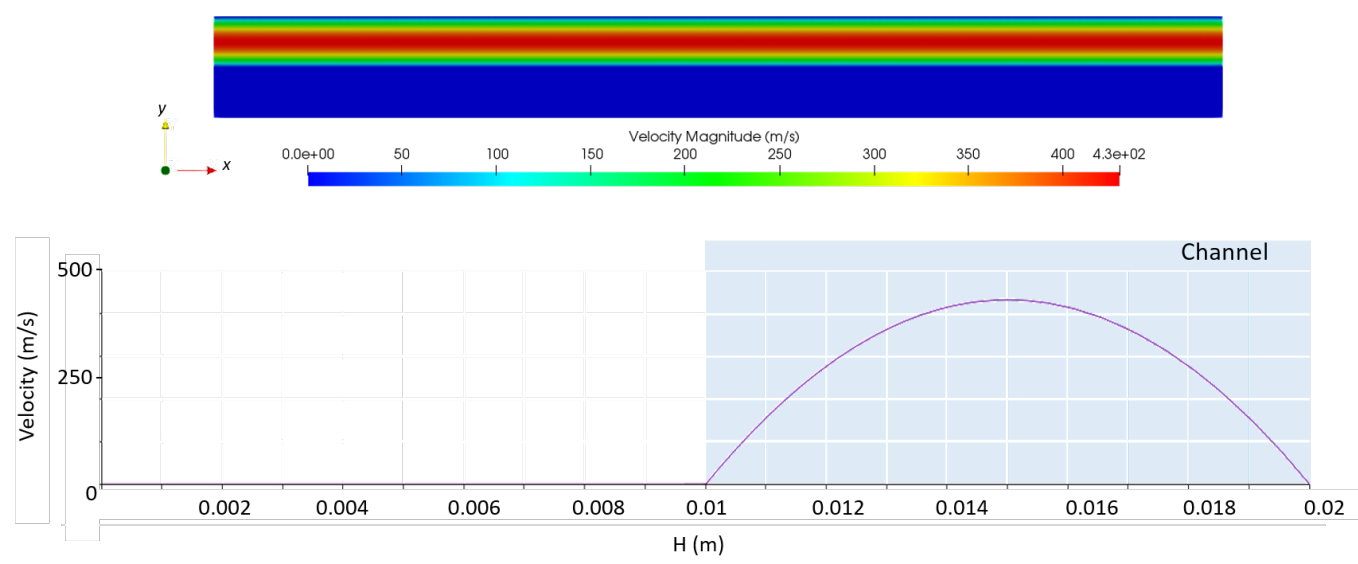

(b) $k_{p m}=1 \times 10^{-10} m^{2}$.

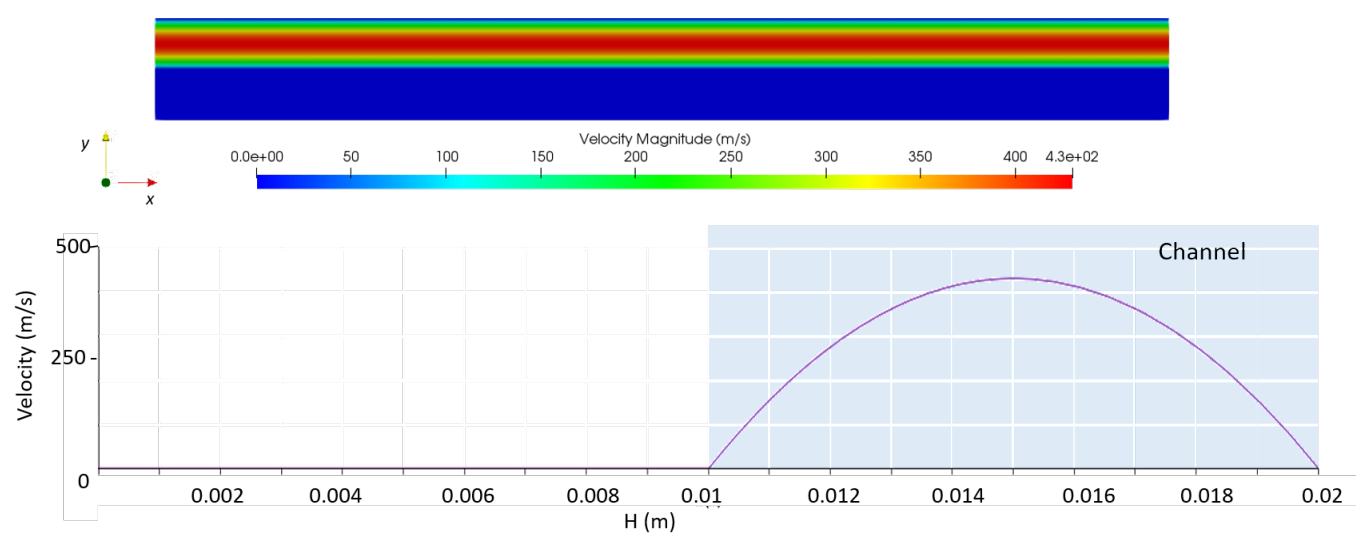

(c) $k_{p m}=1 \times 10^{-12} m^{2}$.

Figure 4.6: Velocity field for three different porous medium permeabilities input and $\Delta P=6,894.76 \mathrm{~Pa}$. Below each velocity field is the velocity profile at the midpoint of the section $(\mathrm{x}=0.1 \mathrm{~m})$. 


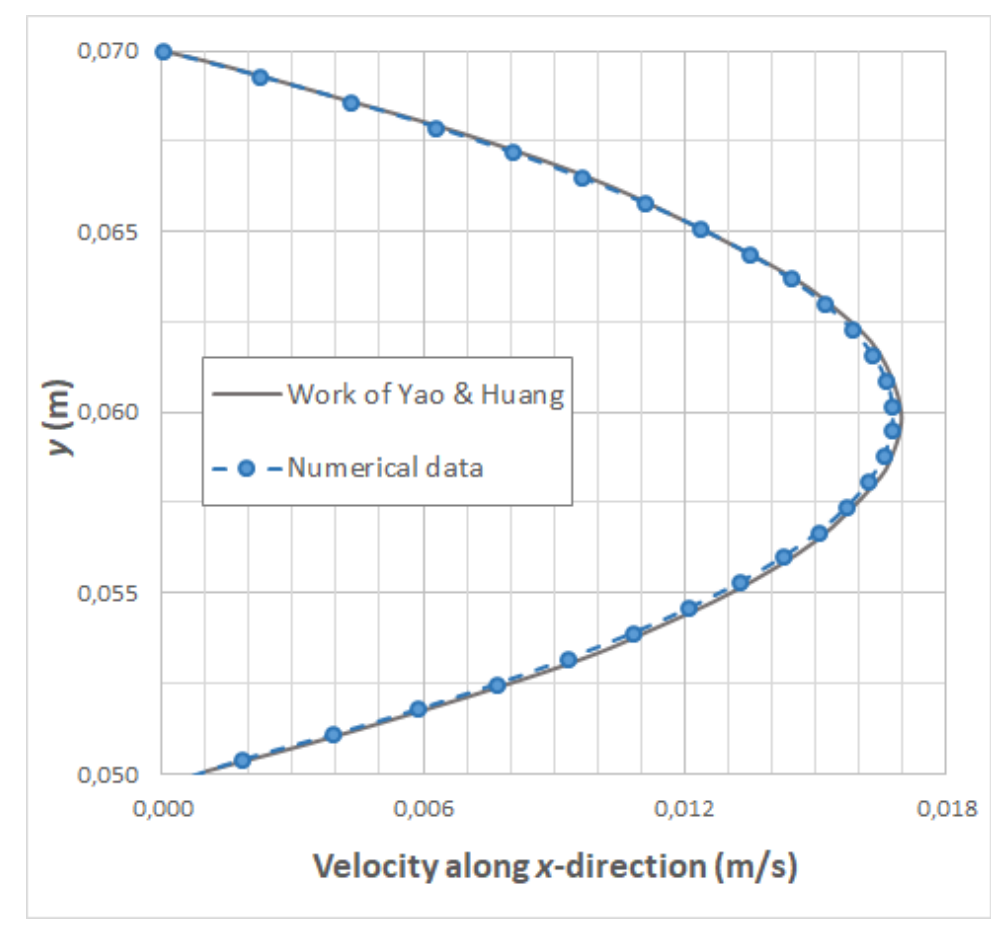

Figure 4.7: Comparison of velocity profile between theoretical and experimental presented by Yao \& Huang, and numerical results of this work.

\subsection{1}

\section{The effect of aperture}

By varying the channel thickness it is possible to study the impact of porous matrix permeability $k_{p m}$ and channel aperture $h$. This free path can be interpreted as a fracture in a reservoir. With this intention, it was created a second mesh, shown in fig. 4.9, similar to the experiment that this study is trying to recover, sketched in fig. 4.8. The no-slip condition was defined in the upper impermeable wall to re-create the conditions of the BJ experimental arrangement. 


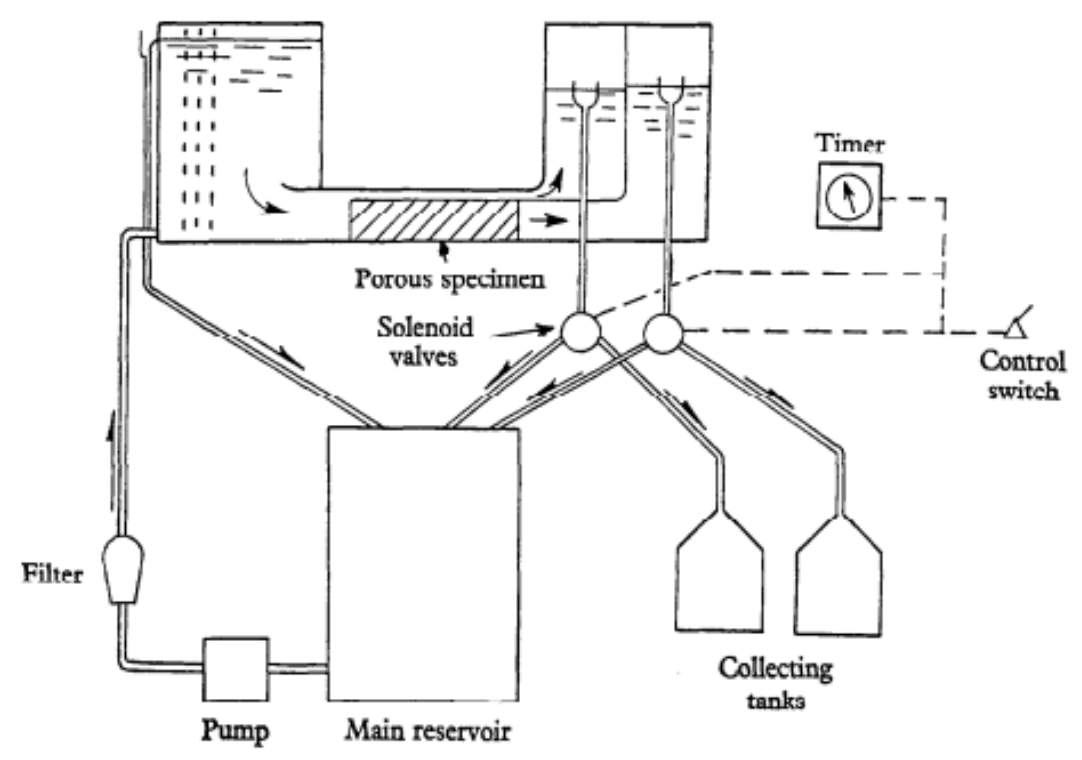

Figure 4.8: Beavers \& Joseph experimental arrangement. From Beavers \& Joseph, 1967.

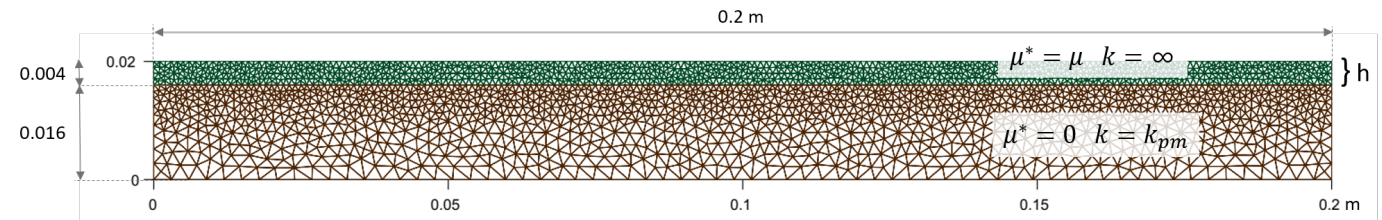

Figure 4.9: Generated mesh to analyze the Beavers-Joseph condition with an smaller aperture, where the green area is the free section and the other is the porous medium.

Figures 4.10 and 4.11 show the results for gaps of $0.0008,0.002$ and 0.004 $\mathrm{m}$. The permeability values $k_{p m}$ were chosen to allow a comparison between experimental and numerical values. It is possible to observe the velocity profile formed inside the channel, blue area, and in the permeable material at the middle of the section, $\mathrm{x}=0.01 \mathrm{~m}$. A parabolic velocity profile is formed in the channel, with a slip velocity at the interface with the permeable wall. This behavior is similar to the one predicted by using the BJ boundary condition.

As expected, the average velocity in the channel and the slip velocity rises as the channel height increases.

For a larger channel as in fig. $4.11 \mathrm{~b}$ with $4 \mathrm{~mm}$, the velocity in the channel is much higher than that of the previous case, fig. 4.11a, and that has an abrupt transfer at the interface and a higher sliding velocity. 


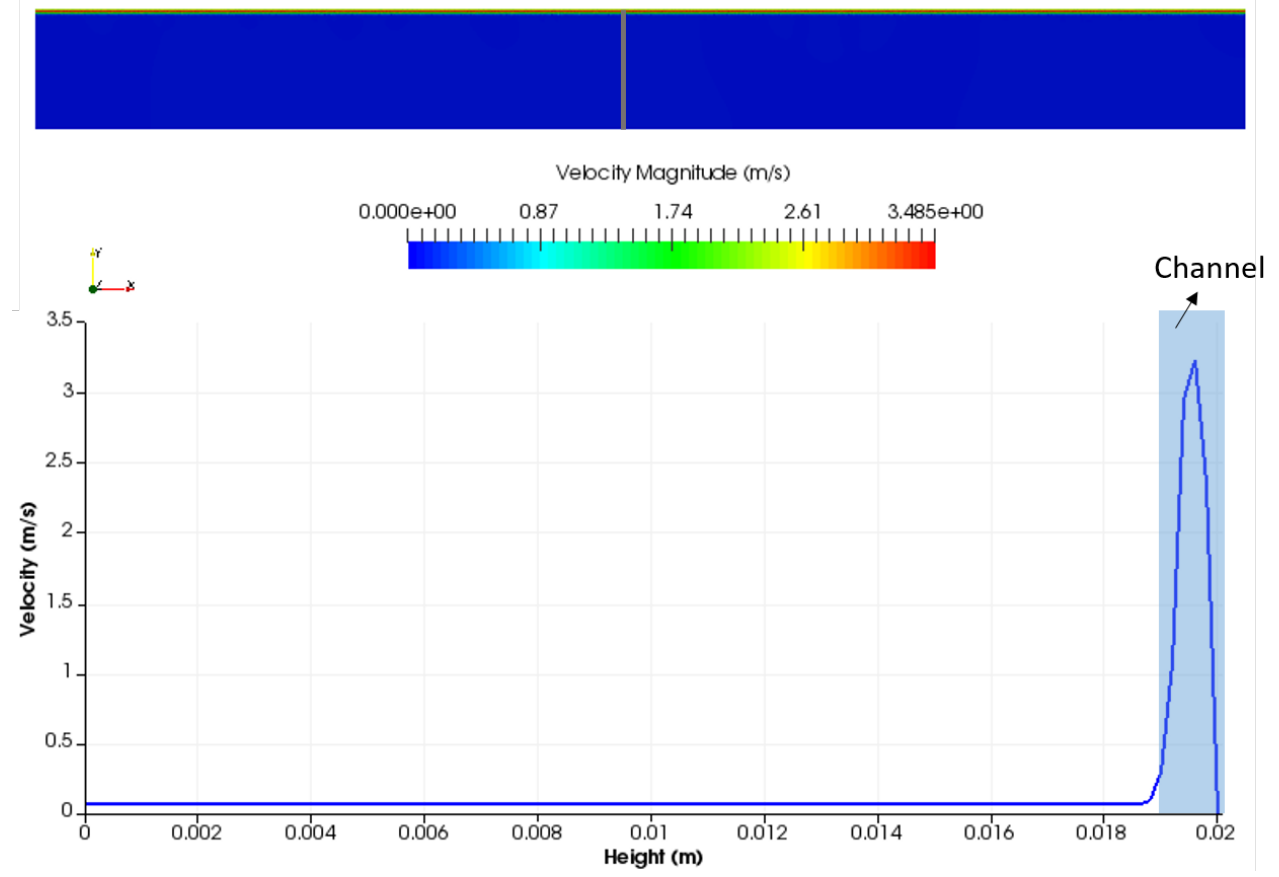

(a) Velocity.
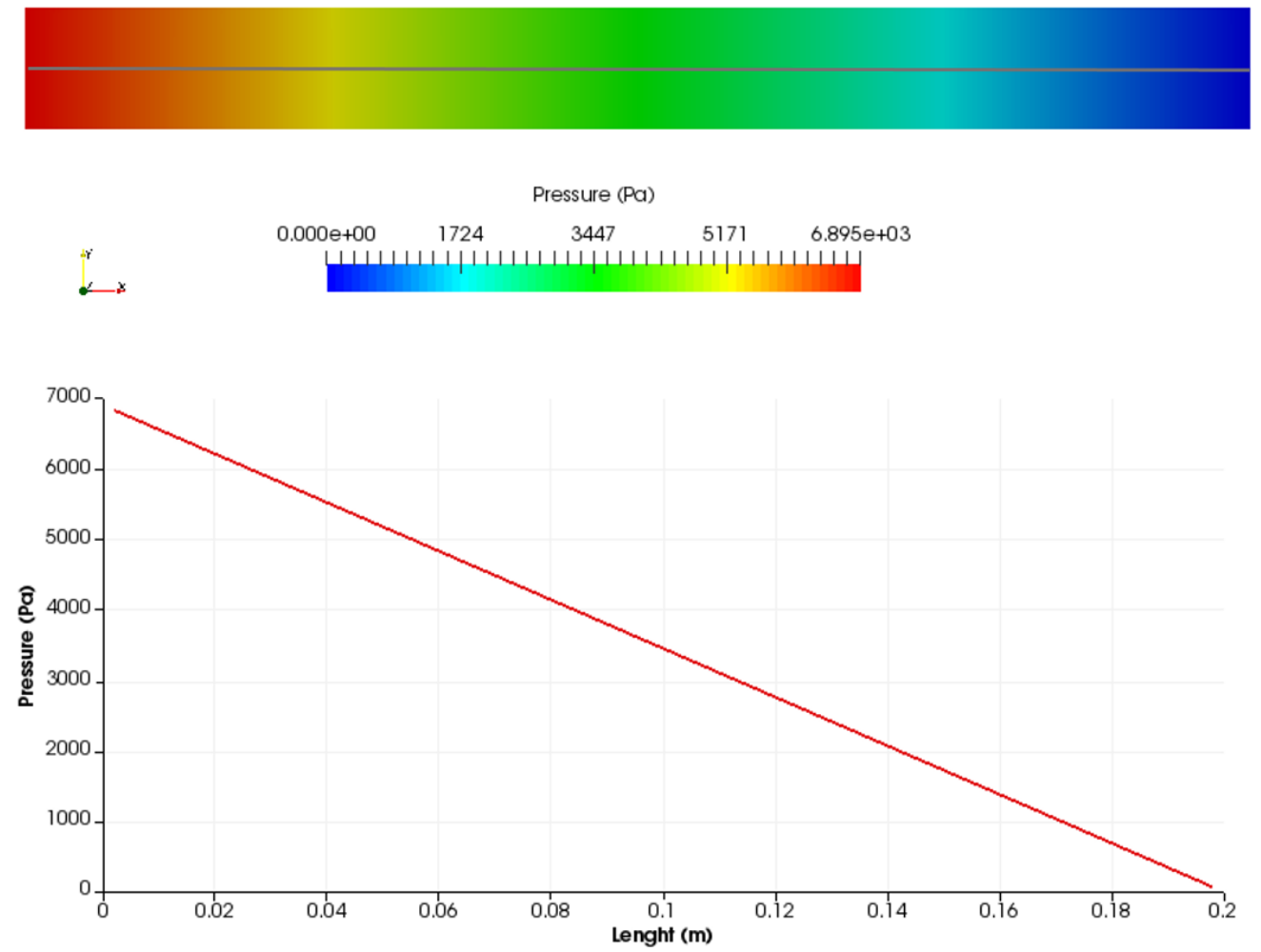

(b) Pressure.

Figure 4.10: Velocity and pressure field for $0.0008 \mathrm{~m}$ aperture with $k_{p m}=1.98$ $\mathrm{x} 10^{-9} \mathrm{~m}^{2}$ and $\Delta P=6,894.76 \mathrm{~Pa}$. On top, 4.10a, are the velocity diagram and profile at $\mathrm{x}=0.1 \mathrm{~m}$. Below this is the pressure diagram and pressure distribution from an inlet to the outlet at $\mathrm{y}=0.01 \mathrm{~m}$. 


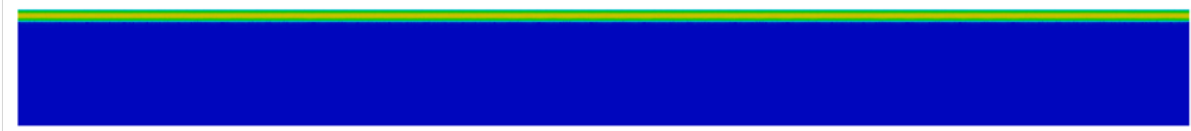



Vebcity Magnitude $(\mathrm{m} / \mathrm{s})$
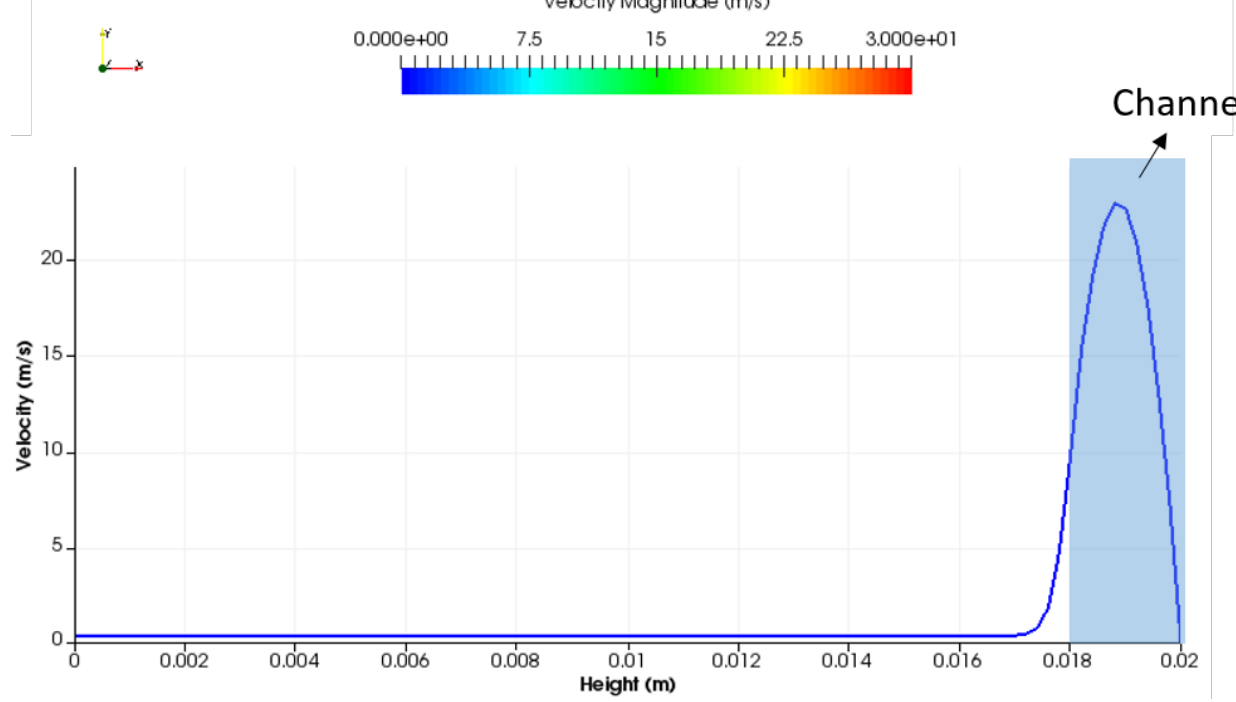

(a) $\mathrm{h}=0.002 \mathrm{~m}$ and $k_{p m}=10^{-8} \mathrm{~m}^{2}$.

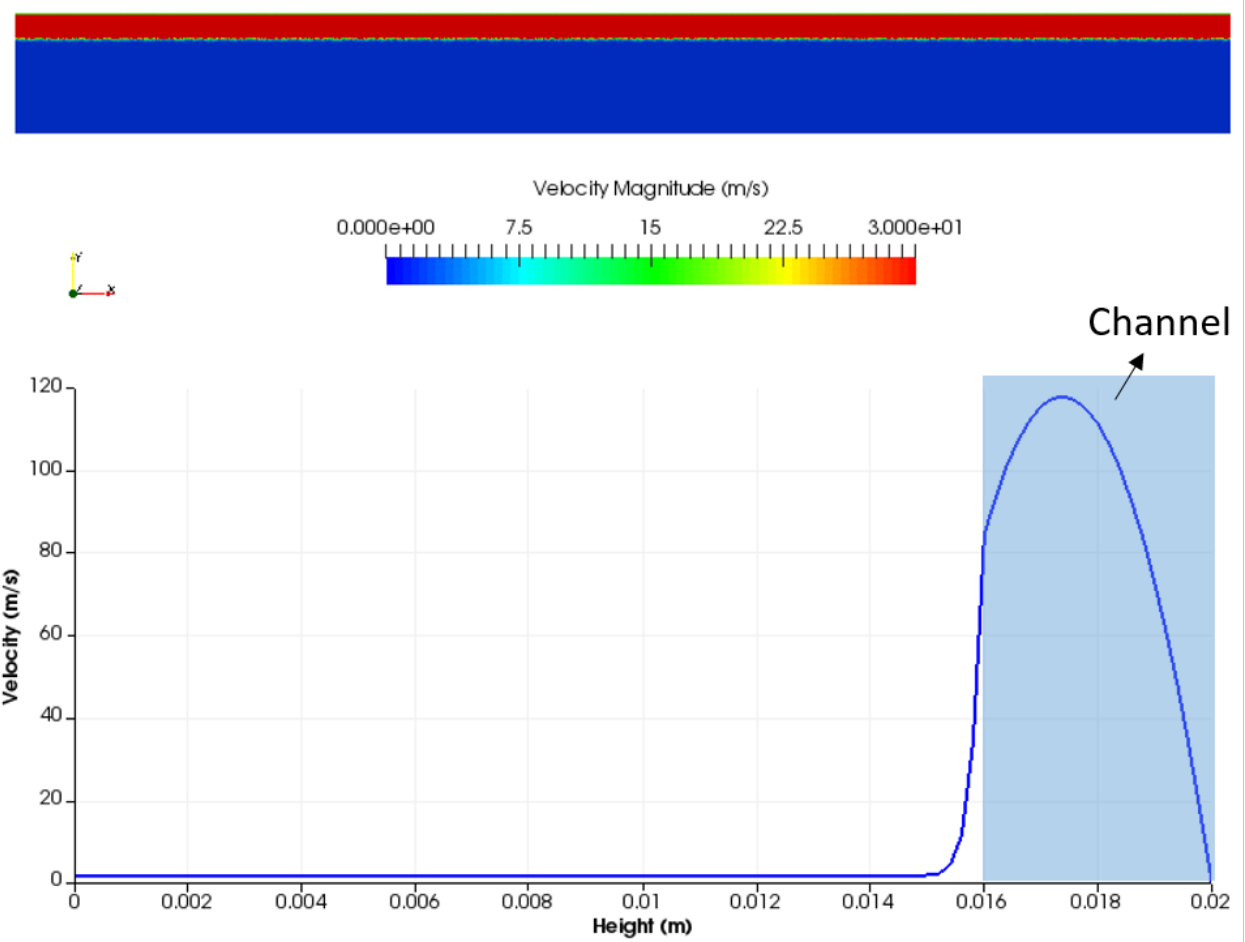

(b) $\mathrm{h}=0.004 \mathrm{~m}$ and $k_{p m}=4.94 \times 10^{-8} \mathrm{~m}^{2}$.

Figure 4.11: Velocity diagram and profile with same velocity scale for two heights of aperture and $\Delta P=6,894.76 \mathrm{~Pa}$. 
In order to analyze the effect of the permeable boundary in the mass flow through the channel, the results of the flow through the channel were evaluated for different heights. This effect is quantified by a parameter $\Phi$, first presented by Beavers and Joseph[5]. Equation 4-2 was defined by them as the relative increase in flow rate due to the presence of a permeable boundary with respect to the flow rate through a channel with impermeable walls:

$$
\Phi=\frac{\left(M-M_{p}\right)}{M_{p}}
$$

where $M$ is the total flux through the channel outlet and $M_{p}$ is the total flux of the outlet in Poiseuille flow problem (impermeable walls).

It is expected an increase in mass flow rate in the free flow region due to velocity slip condition if compared with a no-slip wall with the same channel height $h$ and pressure gradient. Figure 4.12 shows the fractional increase in mass flow rate as a function of $h / \sqrt{(} k)$, considering water as the flowing liquid. The effect of the permeable wall is stronger for narrow gap and high permeability values.

However, it cannot be verified an agreement between the data points (fig. 4.12) and the BJ's experimental flow data (fig. 4.13). The results for the aloxite blocks showed a slight increment in the flow rate due to its permeability, the lowest studied, explained by its small slip velocity. Hence, the largest increment belongs to the material that has the highest permeability, $k_{p m}=8.19 \times 10^{-8} \mathrm{~m}^{2}$.

Thus, a reasonable explanation would be the relevance of the structure of the porous medium in the experiment[3], which Brinkman model considers only permeability $k_{p m}$ as a characteristic of the material. As discussed in section 1.4, the parameter $\alpha$ has a strong relation to the structure of the porous interface and sensitive to microscopic changes in the definition of the interface point [8]. In this way, a value for the parameter $\alpha$ for any curve was not found and the results diverged to a different condition for a given experiment material. 


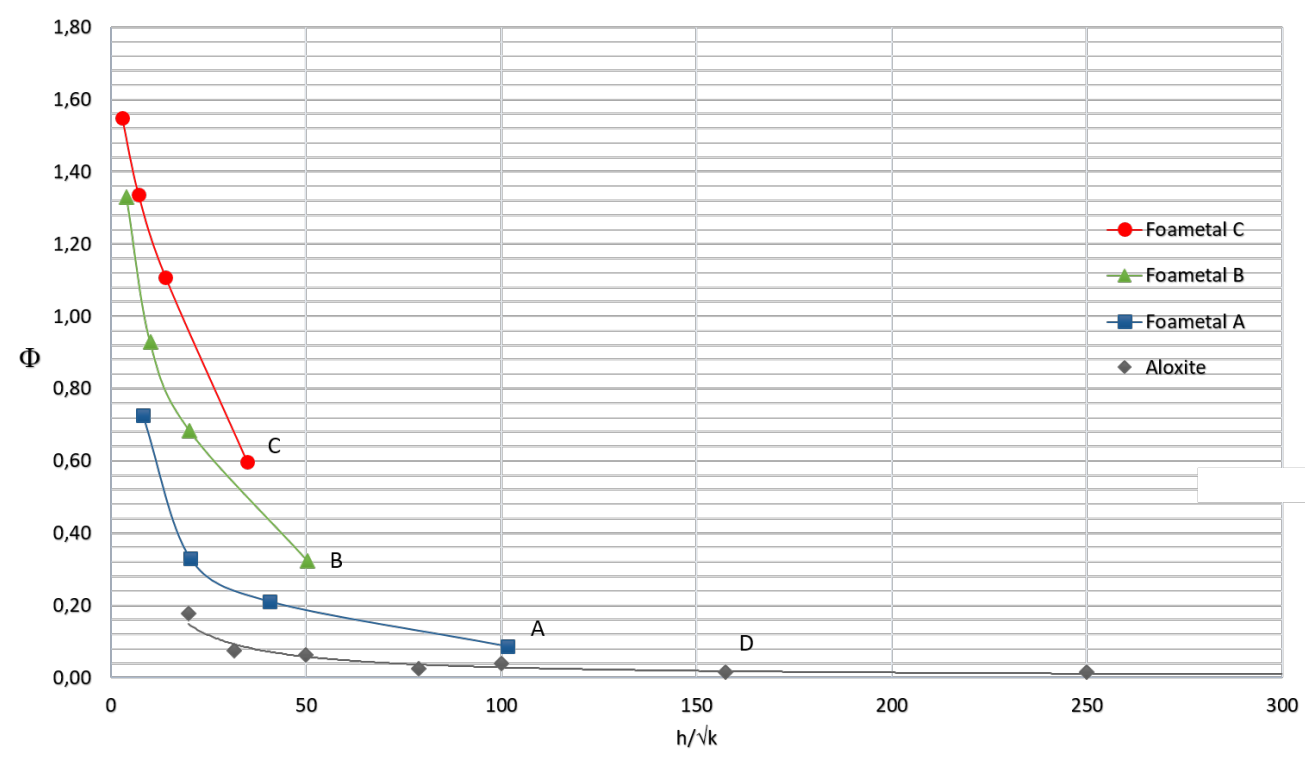

Figure 4.12: $\Phi$ as a function of $h / \sqrt{k_{p m}}$ for the same permeabilities of porous specimens used by Beavers-Joseph. $-k_{p m}=8.19 \times 10^{-8} m^{2} ; \boldsymbol{\Lambda}, k_{p m}=3.94$ $\mathrm{x} 10^{-8} ; \boldsymbol{\square}, k_{p m}=9.68 \times 10^{-9} m^{2} ; \diamond, k_{p m}=1.6 \times 10^{-9} m^{2}$ and $k_{p m}=6.45 \mathrm{x}$ $10^{-10} \mathrm{~m}^{2}$.

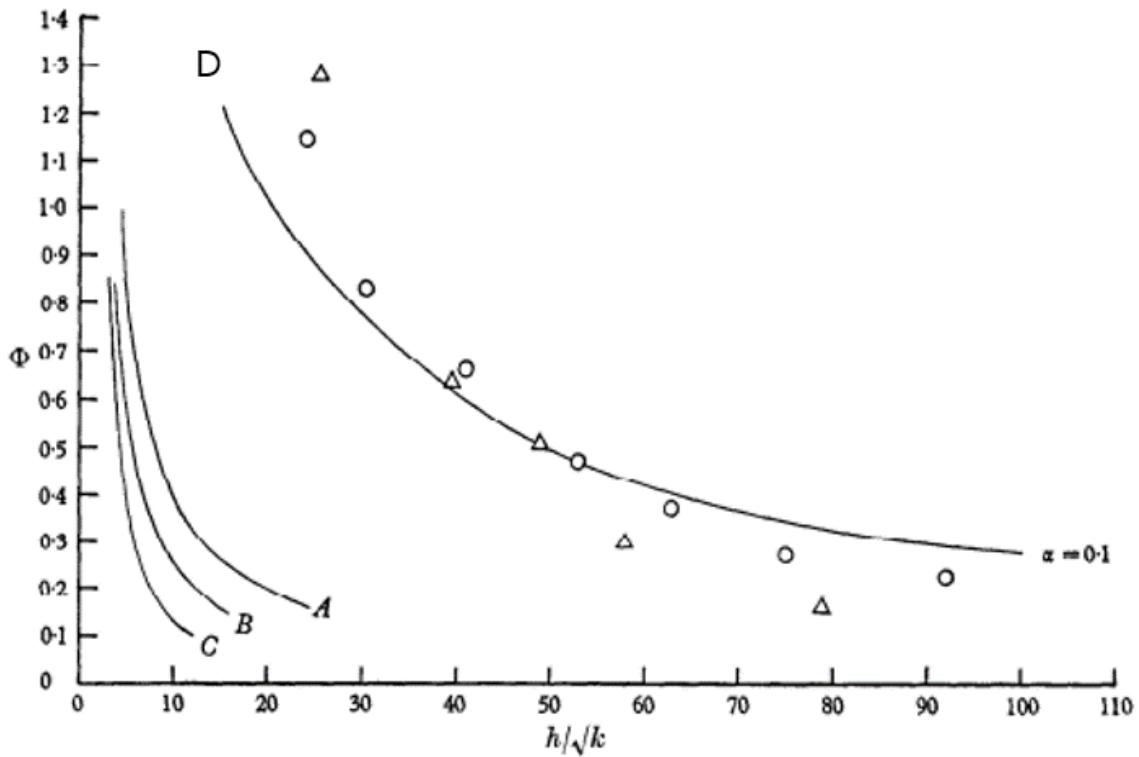

Figure 4.13: $\Phi$ as a function of $h / \sqrt{k_{p m}}$. C, $k_{p m}=8.19 \times 10^{-8} m^{2} ; \mathrm{B}, k_{p m}=$ $3.94 \times 10^{-8} ; \mathrm{A}, k_{p m}=9.68 \times 10^{-9} m^{2} ; \mathrm{D}, k_{p m}=1.6 \times 10^{-9} m^{2}$ and $k_{p m}=6.45$ $\mathrm{x} 10^{-10} \mathrm{~m}^{2}$. From Beavers \& Joseph, 1967.

\subsection{2}

\section{Comparison Beavers-Joseph boundary condition}

In this section, we analyzed how close the flow fluid, predicted by solving Brinkman's model, follows the BJ boundary condition: 


$$
-\left.\frac{d u}{d y}\right|_{i n t}=\frac{\alpha}{\sqrt{k_{p m}}}\left(u_{B}-u_{D}\right) .
$$

where $u_{B}$ is the velocity at the interface, $u_{D}$ is the Darcy velocity of the fluid in the porous medium, $k_{p m}$ the permeability of the material and $\alpha$ is a dimensionless parameter, which as suggested by Beavers and Joseph, is a function of the porous medium structure.

Here, four channel thicknesses, $0.8 \mathrm{~mm}, 2 \mathrm{~mm}, 4 \mathrm{~mm}$ and $1 \mathrm{~cm}$, and different permeabilities for the permeable material $k_{p m}$ were used. The results obtained in this test are presented in fig. 4.14, as the parameter $Z$, definided as:

$$
Z=\frac{\frac{d u_{B}}{d y}}{u_{B}-u_{D}} .
$$

The BJ condition states that $Z$ is inversely proportional to $k_{p m}$. the value of $Z$ is plotted as a function of permeability for different channel heights in fig. 4.14. A curve fit with a power law relation is used, i.e. $Z \approx k_{p m}^{n}$. The parameter $n$ for each case is presented in tab. 4.3.

Only the data obtained for a channel with a thickness of $0.8 \mathrm{~mm}$ shows the same dependence on $k_{p m}$ as the Beavers-Joseph boundary condition. It is important to note that this was the channel height used in their experiment. For the other channel heights, the data present a better fit for other permeability power values.

\begin{tabular}{|rrr|}
\hline \multicolumn{1}{c}{$\mathrm{h}(\mathrm{m})$} & \multicolumn{1}{c|}{$\boldsymbol{\alpha}$} & $\mathrm{n}$ \\
\hline 0,0008 & 0,73 & 0,45 \\
0,002 & 16,73 & 0,28 \\
0,004 & 824,00 & 0,04 \\
0,01 & $1,93 \mathrm{E}-05$ & 1,00 \\
\hline
\end{tabular}

Table 4.3: Curve adjustment for boundary condition equation.

\section{2 .3}

\section{Effect of model parameter $\mu^{*}$ in the flow prediction}

In the results shown up to now, the value of the parameter $\mu^{*}$ is set to be equal the fluid viscosity in the channel, $\mu^{*}=\mu$, and arbitrarily to zero in the permeable region, $\mu^{*}=0$. In order to evaluate the effect of this approximation in the predictions, we obtained flow fields using $\mu^{*}=\mu$ for the entire flow 


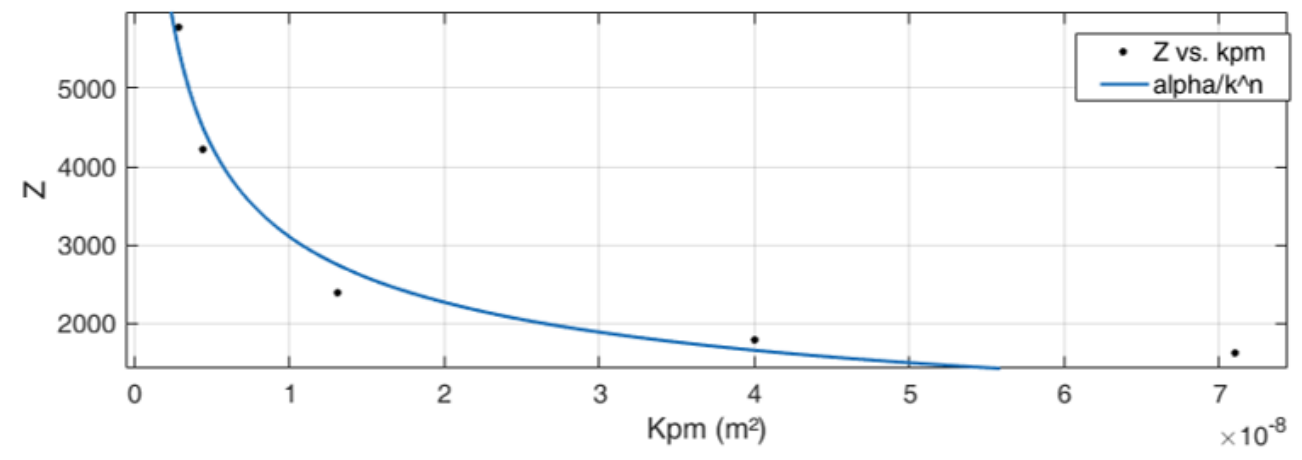

(a) $h=0.0008 \mathrm{~m}$.

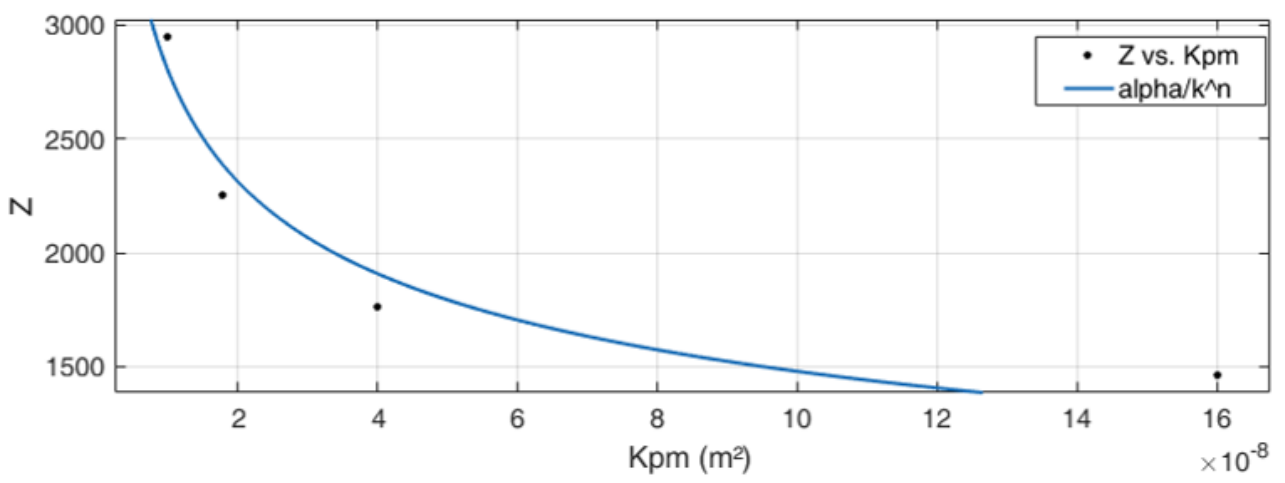

(b) $h=0.002 \mathrm{~m}$.

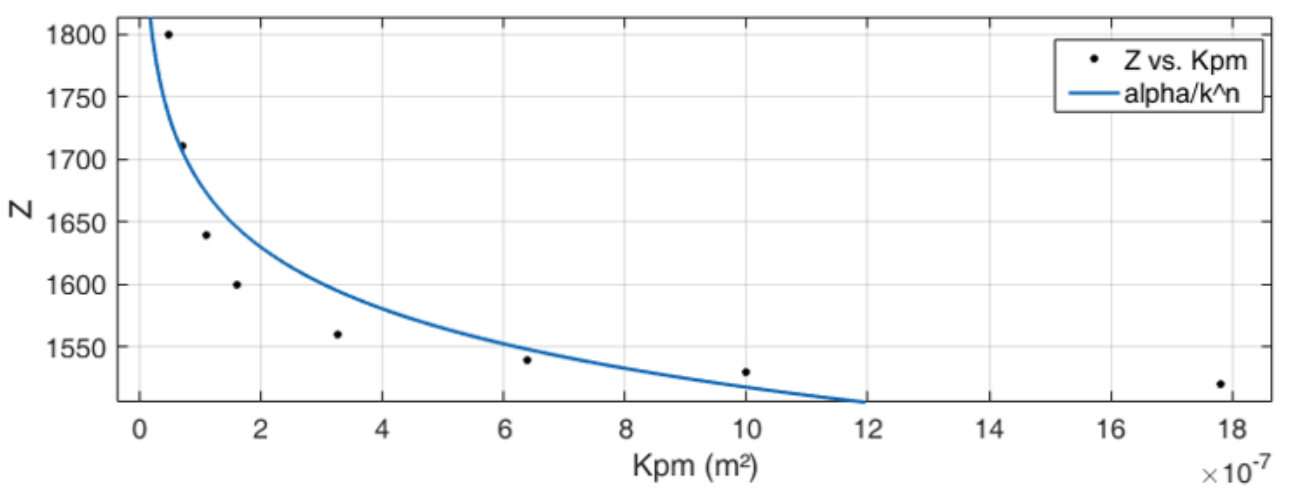

(c) $h=0.004 \mathrm{~m}$.

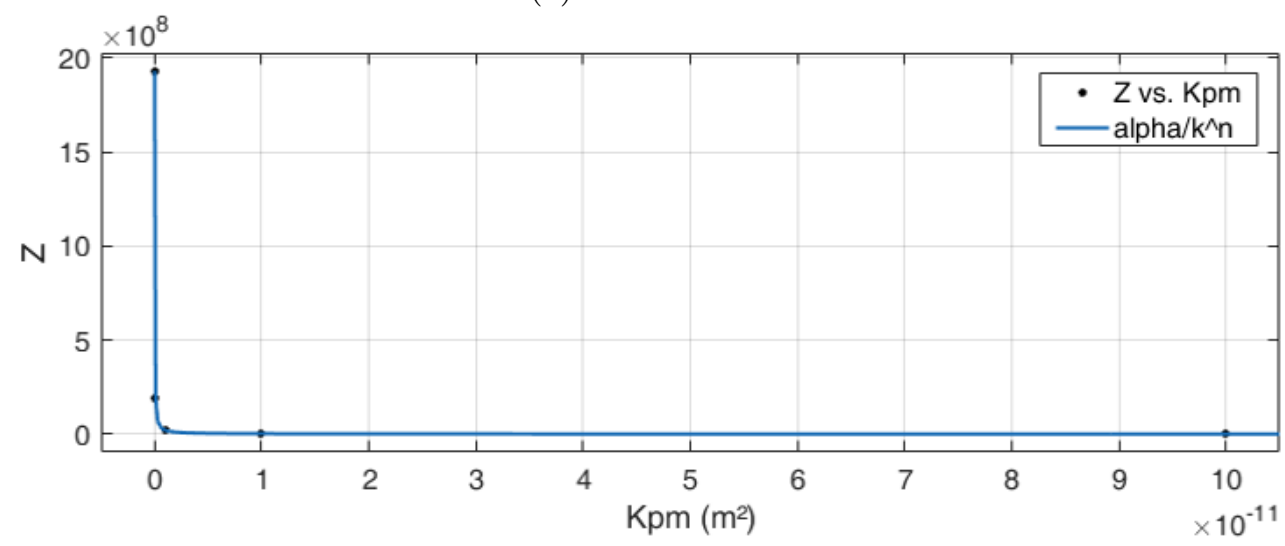

(d) $h=0.01 \mathrm{~m}$.

Figure 4.14: Curve adjustment for various apertures. 
domain. The comparison is shown in figs. 4.15 and 4.16, for a channel with 2 $\mathrm{mm}$ height, a pressure gradient of $6,894.76 \mathrm{~Pa}$ and permeability of the porous region of $k_{p m}=1 \times 10^{-12} m^{2}$.

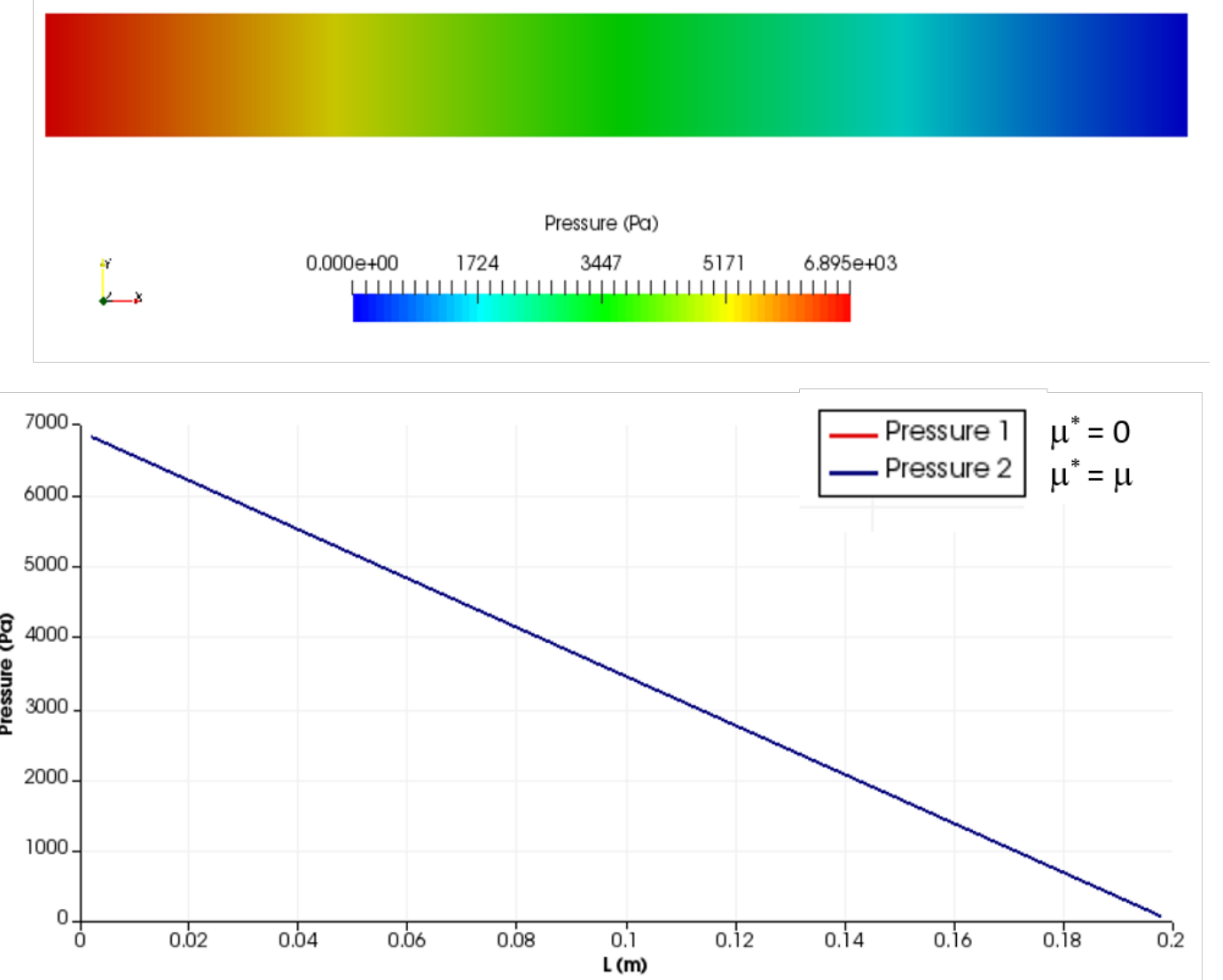

Figure 4.15: Stability model test: Pressure.

Figure 4.16 shows that the velocity profile is almost insensitive to the value of $\mu^{*}$. The flows rates through the porous layer $Q_{p m}$ differ by only $1.79 \%$ for the highest permeability $\left(k_{p m}=1 \times 10^{-10} m^{2}\right)$ and there is virtually no significant difference for lower permeability values, as shown in tab. 4.4.

\begin{tabular}{|c|c|ccc|ccc|r|}
\cline { 3 - 8 } \multicolumn{2}{c|}{} & \multicolumn{3}{c|}{$\mathrm{k}=\mathrm{k}_{\mathrm{pm}} \quad \dot{\mu}=0$} & \multicolumn{3}{c|}{$\mathrm{k}=\mathrm{k}_{\mathrm{pm}} \quad \dot{\mu}=\mu$} & \multicolumn{1}{c|}{} \\
\hline$\Delta \mathrm{P}(\mathrm{Pa})$ & $\mathrm{K}_{\mathrm{pm}}\left(\mathrm{m}^{2}\right)$ & $\mathrm{Q}_{T}$ & $\mathrm{Q}_{\mathrm{h}}$ & $\mathrm{Q}_{\mathrm{pm}}$ & $\mathrm{Q}_{T}$ & $\mathrm{Q}_{\mathrm{h}}$ & $\mathrm{Q}_{\mathrm{pm}}$ & $\mathrm{Q}_{\mathrm{pm}}$ Diff. \\
\hline \multirow{6}{*}{6894,76} & $1,00 \mathrm{E}-10$ & 0,02267 & 0,02256 & $1,12 \mathrm{E}-04$ & 0,02266 & 0,02255 & $1,10 \mathrm{E}-04$ & $1,79 \%$ \\
& $1,00 \mathrm{E}-11$ & 0,02249 & 0,02248 & $1,12 \mathrm{E}-05$ & 0,02249 & 0,02248 & $1,12 \mathrm{E}-05$ & $0,00 \%$ \\
& $1,00 \mathrm{E}-12$ & 0,02247 & 0,02247 & $1,12 \mathrm{E}-06$ & 0,02247 & 0,02247 & $1,12 \mathrm{E}-06$ & $0,00 \%$ \\
& $1,00 \mathrm{E}-13$ & 0,02247 & 0,02247 & $1,12 \mathrm{E}-07$ & 0,02247 & 0,02247 & $1,12 \mathrm{E}-07$ & $0,00 \%$ \\
& $1,00 \mathrm{E}-14$ & 0,02247 & 0,02247 & $1,12 \mathrm{E}-08$ & 0,02247 & 0,02247 & $1,12 \mathrm{E}-08$ & $0,00 \%$ \\
& $1,00 \mathrm{E}-30$ & 0,02247 & 0,02247 & $1,12 \mathrm{E}-24$ & 0,02247 & 0,02247 & $1,12 \mathrm{E}-24$ & $0,00 \%$ \\
\hline
\end{tabular}

Table 4.4: Difference between methods. 

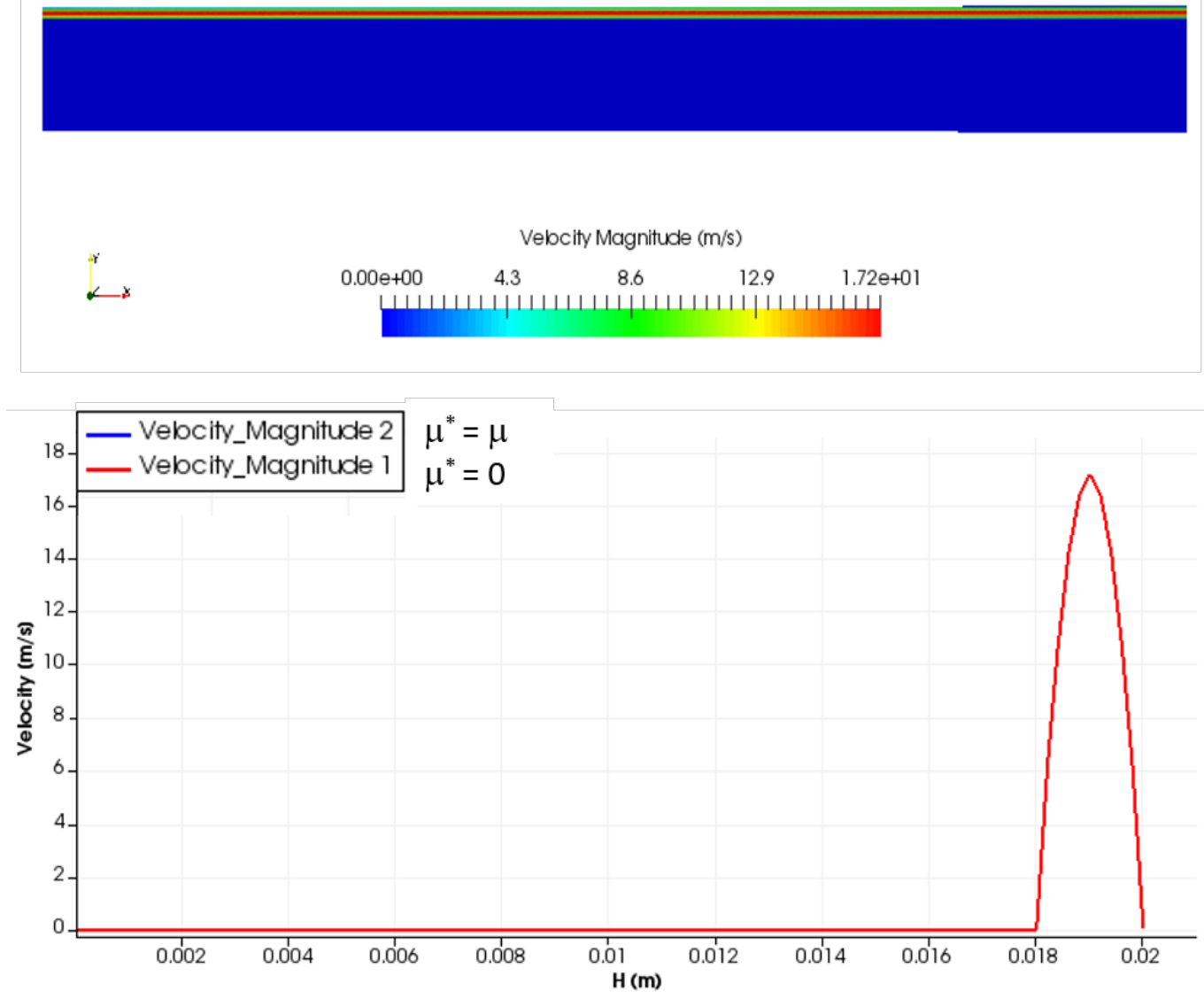

Figure 4.16: Stability model test: Velocity.

The analysis of the model at solid/void interface reported similar experimental predictions. The simulation tool using the Brinkman equation was validated and was capable to reproduce the behavior described by the BeaversJoseph boundary condition. This comparison also recovers the same boundary equation of BJ for the same channel height $(\mathrm{h}=0.0008 \mathrm{~m})$. Once it is shown that the model is solid, it is possible to apply the simulation tool on a piece of vuggy porous media. The next chapter presents modeling and simulation in segments of digital rocks with the purpose of developing a methodology to estimate equivalent permeability. 


\section{Flow through vuggy porous media}

This chapter presents the results of this work concerning the analysis of flow through porous media with large vugs. The expected result of the project is a methodology to estimate equivalent permeability in different geometries with heterogeneous characteristics. The cores tested were similar to those found in the real case of carbonate reservoirs, that is, with the presence of cavities that may or may not be interconnected. Hence, it is necessary to define a virtual model that represent carbonate samples.

In order to evaluate the flow through the core sample, a methodology was created to obtain the two-dimensional geometry model from two core samples. Three tomographic images of each core sample were selected and virtually modified to expand the macroporosity area studied in this analysis. The use of images from more than one sample and the manipulated expansion of macroporosity also permit the study of the influence of the vug shape, size, and distribution in flux. Then, the simulation tool was used to determine the equivalent permeability of each geometry. Finally, the results from the numerical analysis based on vuggy porous media are presented.

\section{1}

\section{Core Samples}

Two plugs of coquinas withdrawn from Morro do Chaves Formation located in the Sergipe-Alagoas Basin were used as the porous media for this part of the analysis. Samples from this formation are considered analogous to the reservoir rocks of the pre-salt layer of the Campos and Santos Basins because they date from the same period of geological formation[46].

The collected material was submitted to a micro-tomography of X-ray as well as the determination of the absolute permeability and porosity of the samples as a function of the confinement pressure. Table 5.1 contains a summary of the core samples properties. 


\begin{tabular}{ccccccc}
\hline Sample & $\begin{array}{c}\text { Diameter } \\
(\mathbf{m m})\end{array}$ & $\begin{array}{c}\text { Lenght } \\
\mathbf{( m m}\end{array}$ & $\begin{array}{c}\text { Mass } \\
\mathbf{( g )}\end{array}$ & $\begin{array}{c}\text { Porous } \\
\text { Volume } \\
(\mathbf{m l})\end{array}$ & Porosity (\%) & $\begin{array}{c}\text { Absolute } \\
\text { permeability (mD) }\end{array}$ \\
\hline AM05 & 38,14 & 88,03 & 241 & 6,72 & 6,7 & 0,21 \\
AM08 & 37,86 & 91,05 & 235,1 & 12,53 & 12,2 & 0,97 \\
\hline
\end{tabular}

Table 5.1: Core plug geometry details and routine core analysis results for coquinas samples.

Lima et al. [38] analyzed the pore size distribution of six samples extracted from the same formation and showed that most of the pores in these materials are qualitatively classified as macropores according to fig. 5.1.

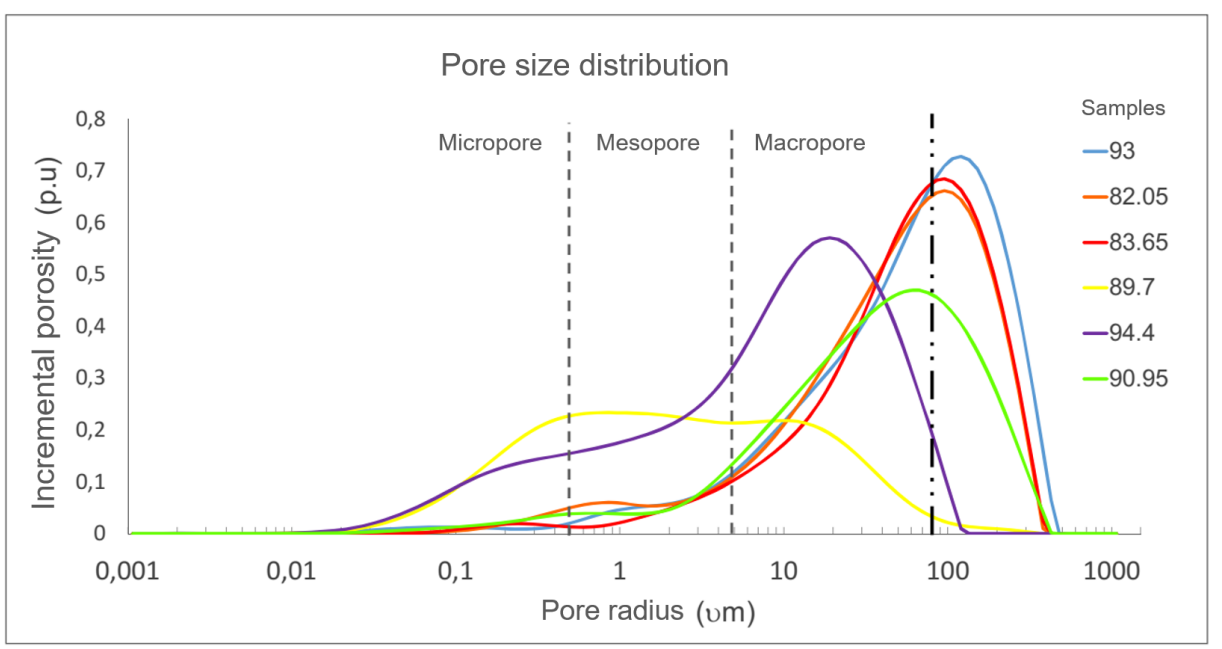

Figure 5.1: Pore size distribution for coquinas samples. Adapted from Lima et al.,2018.

In order to make possible a determination of the porous geometry, the two-dimensional $\mu \mathrm{CT}$ images of the first sample (AM08) extracted in the vertical direction and the other (AM05) in the horizontal direction were analyzed. Figures 5.2 and 5.3 show the digital rock reconstruction of the void spaces where it can be observed a visible stratigraphic difference between them. The complexity and high characteristic heterogeneity of this type of rocks are most apparent by the difference between the directions of the cut used during the extraction of each core sample. Also, it is possible to note in the tomography image in fig. 5.2 structures formed by shell fragments whit a concave format and white color. 

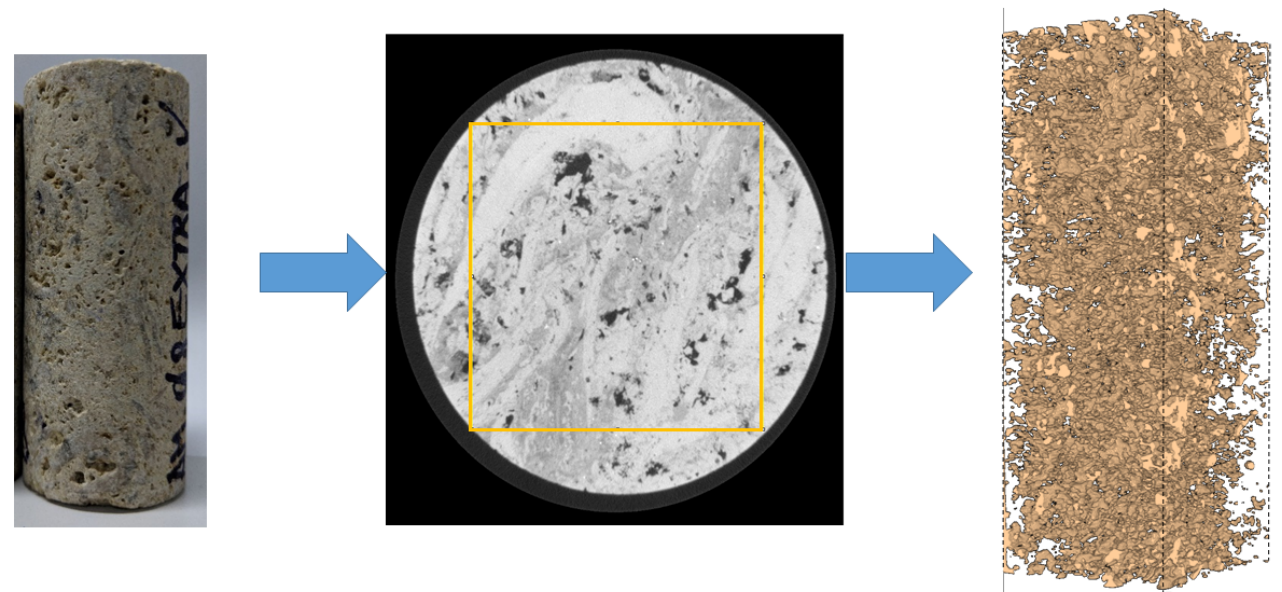

Figure 5.2: Construction of the virtual model for sample 1.
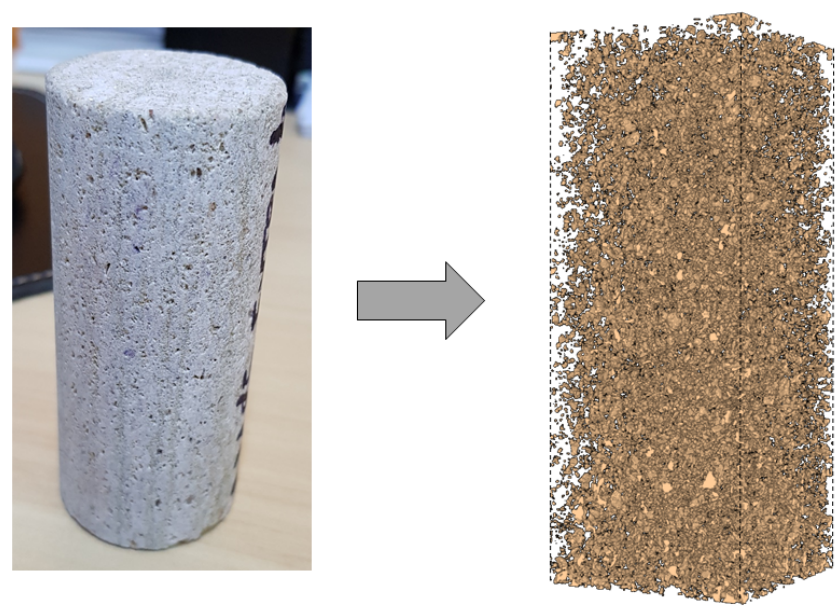

Figure 5.3: Virtual model for sample 2.

\subsection{1}

\section{Model of a rock sample}

This study proposes the acquisition and processing of $2 \mathrm{D}$ images of coquinas samples obtained by MRX for analysis of the internal microstructure (pore space morphology and connectivity) as well as for the evaluation of rock heterogeneity and use of the porous structure for simulations flow. The image acquisition was performed by the Laboratory of Digital Microscopy of the Department of Chemical Engineering and Materials of PUC-Rio. 


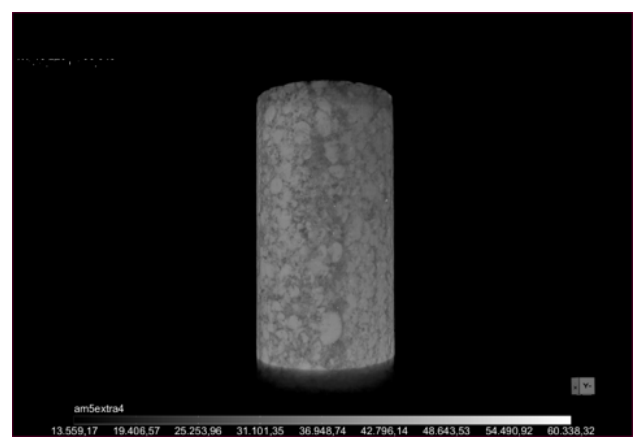

(a) Sample Am05.

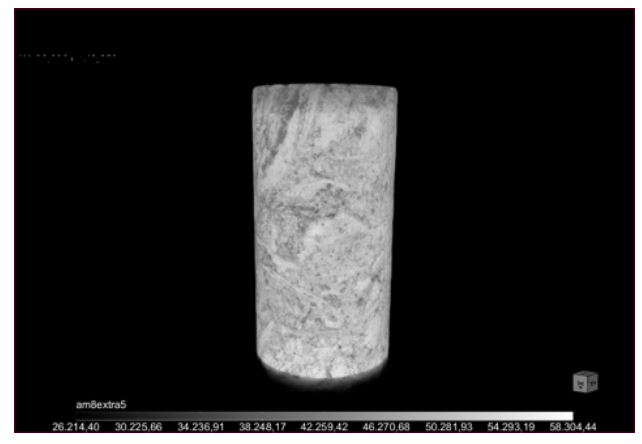

(b) Sample Am08.

Figure 5.4: X-ray microtomography of coquine samples: 3D images of the samples.

The technique is based on the principle of X-ray imaging, in which several $\mathrm{X}$-rays of the analyzed object are recorded at different angles of rotation. These images are used to compose a representation in three dimensions through computational processing. The sample is positioned between the Xray emission source and the detector and is then rotated to acquire projections at various positions. These projections are proportional to the amount of radiation arriving at the detector, which varies according to the different X-ray attenuation coefficients of each material.

The reconstructed 3D images were processed using the software Dragonfly v3.5 (ORS) and the processing of the images contemplated the application of Non-Local Means digital filter for noise reduction. The two-dimensional cutsegmentation process of each sample and the representation of the volume of digitally processed data is shown in figs. 5.4 and 5.5. The first sample, Am08, has in digital format 2,056 slices with $1001 \times 1024$ pixels each; and the second sample, Am05, has 2,011 slices with 999 x 1024 pixels each.

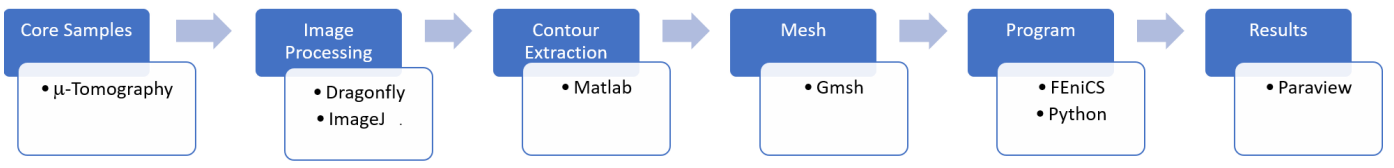

Figure 5.6: Procedure used for a 2D simulation in representative geometries based on $\mu$-tomography images.

For the purpose of simulating a 2D flow through carbonate porous geometry, the procedure summarized in fig. 5.6 was used. The flowchart of fig. 5.7 illustrates the steps that each software was used in image processing. In order to construct a $2 \mathrm{D}$ model that represented a section of carbonate, first, a slice of the microtomography was chosen, as shown in fig. 5.10a, and this image was further processed in the ImageJ software, which is a popular open-source for scientific image processing [47]. 


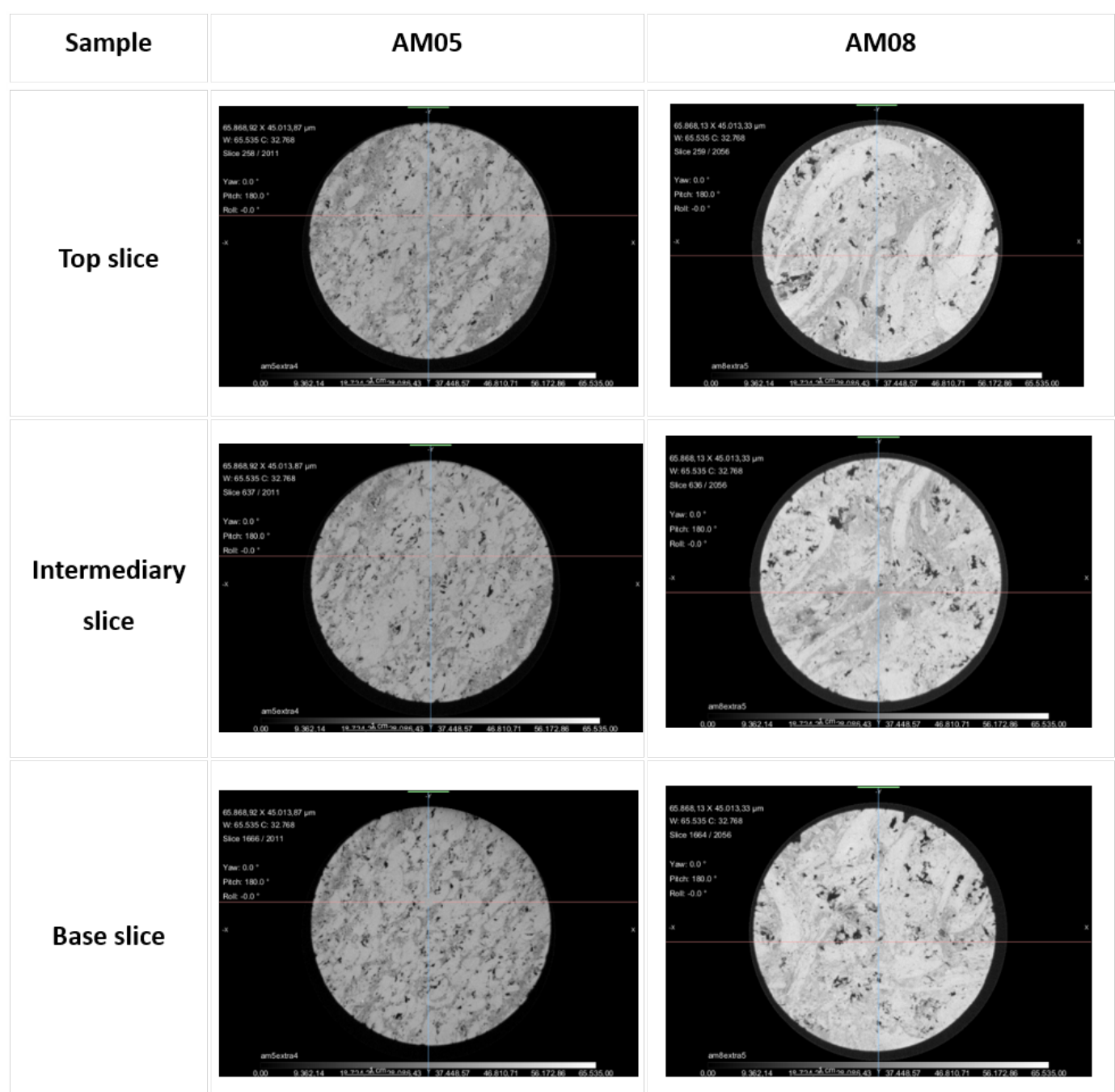

Figure 5.5: X-ray microtomography of coquine samples: slices extracted at the top, middle and bottom of the rocks.

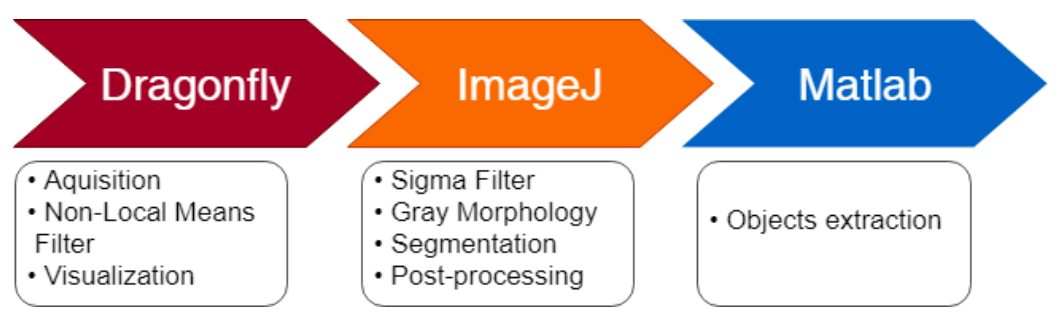

Figure 5.7: Flow chart of digital image processing.

To understand the effect of the size of the cavities on the flow, the methodology presented in the previous sub-section was used in the calculation of the equivalent permeability for different geometries. For this, ImageJ software was chosen because it has high performance and practicality in image processing. The open source software allows the development of various plug-ins, adding different applications to the software. The visualization and quantification of two-dimensional images of this software are efficient and ro- 
bust enough to process the tomographic images of this work.

In this software, a sequence of commands was applied in fig. 5.8a in order to separate the object of interest, which is the vug region. The image file type choose was 8 bits allowing 256 shades of gray. The Sigma Filter and Gray Morphology were applied to soften the noise and smooth the borders.

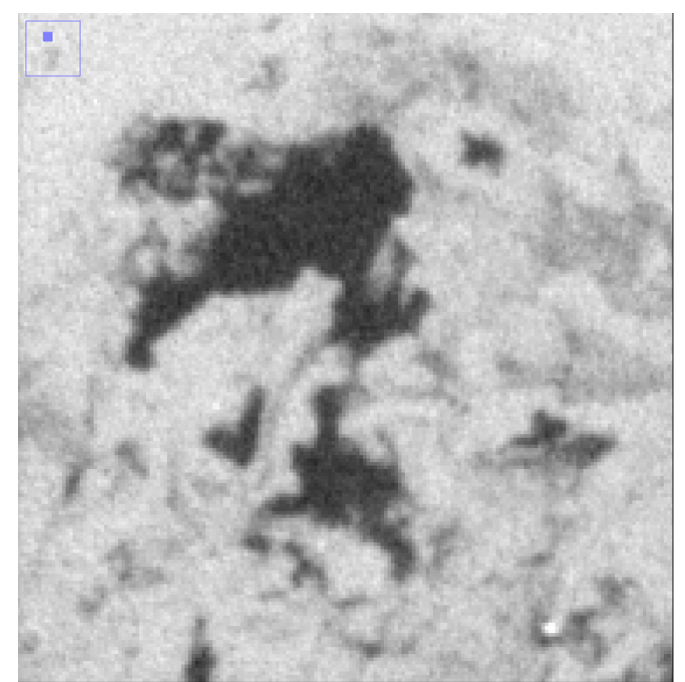

(a) Original

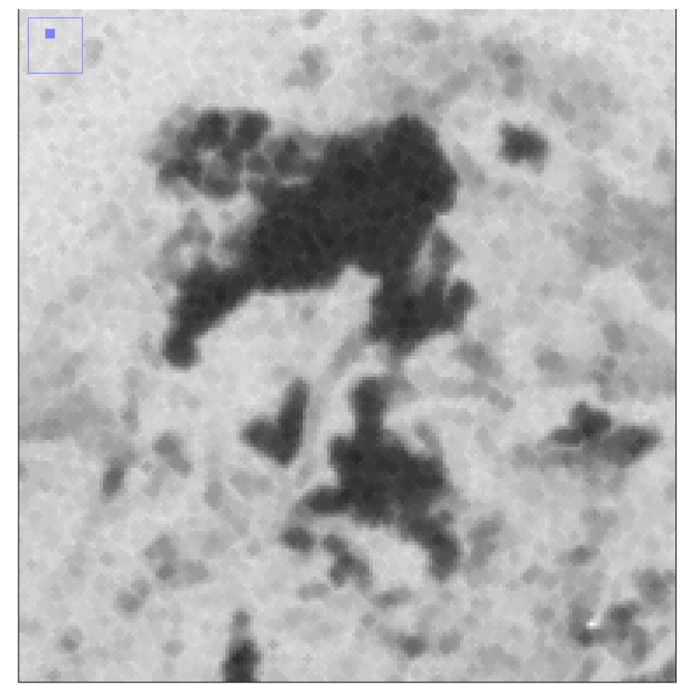

(b) Processed

Figure 5.8: Pre-processing of a slice of the upper part (slice 1) of sample 1.

Figure 5.8b was binarized and segmented into two regions (one containing vugs and one containing porous matrix) as shown in fig. 5.9a. Figure 5.9b illustrates the holes filled to not permit the existence of a porous matrix inside a vug what difficult the mesh creation. Figure 5.10b shows the segmented rock, where the identified holes were filled with black color. The image was cropped in a square format to create the domain of interest and facilitate the definition of the boundary conditions at the simulation tool. Before the attributes extraction, the noise was reduced to eliminate thin or very small objects, less than an element size used in the mesh parameters which an element cannot be created inside this tiny hole.

Then, the contours of the processed image were identified, as presented in fig. 5.10c, with a Matlab based program developed during this study. Appendix A.2 contains the full implementation of the contours extraction code. A CAD geometry generated for this code was imported to the Gmsh software. This application is a generator of three-dimensional finite element mesh[48]. 


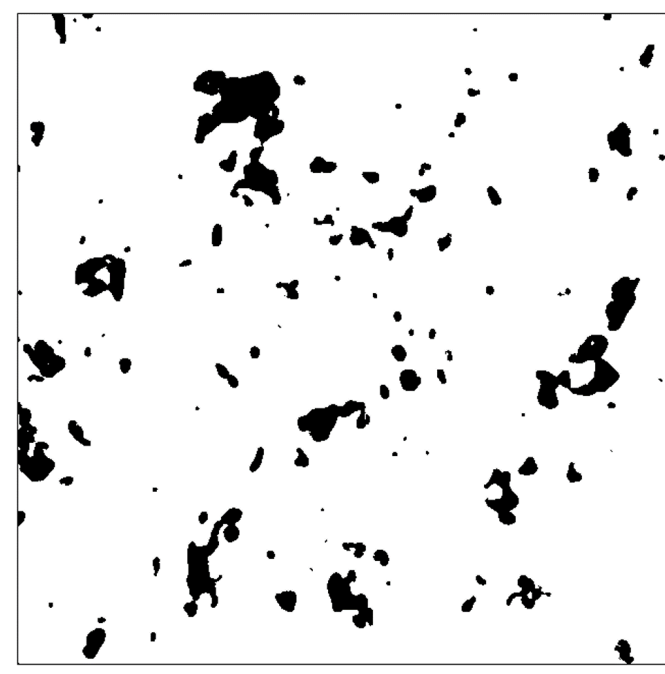

(a) Threshold

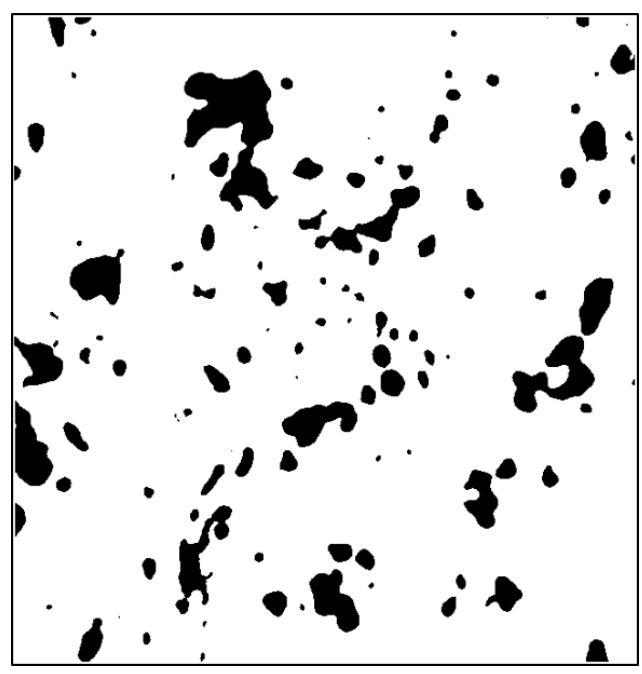

(b) Fill Holes

Figure 5.9: Segmentation of a slice of the upper part (slice 1) of sample 1.

As a final procedure, a two-dimensional mesh with two distinct regions was constructed using Gmsh software based on the points cloud extracted in Matlab, as shown in fig. 5.11. This discretization of a rigid porous matrix is a non-structured mesh composed by triangular elements. The mesh used in this work was not uniform, in this way, a smaller element size was employed near porous matrix-void interface to possibility a better accuracy of the model analysis at the transition of sub-domains.

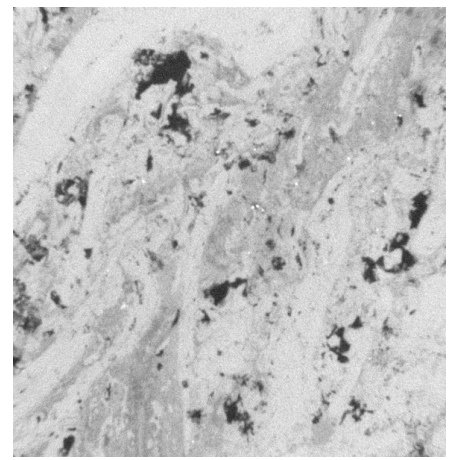

(a) $\mu \mathrm{CT}$ image

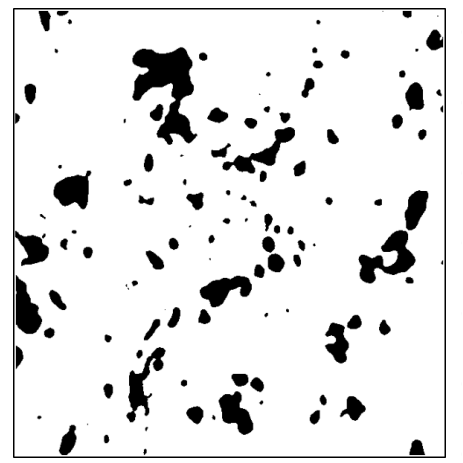

(b) Image processing

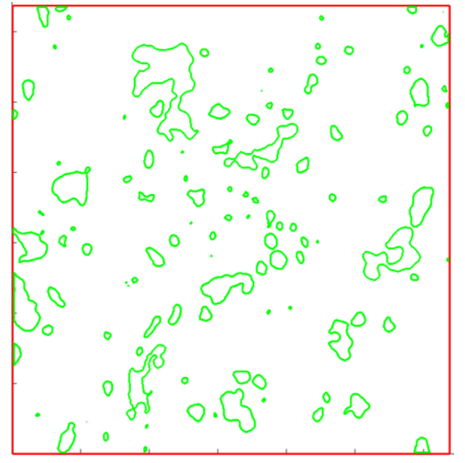

(c) Contour extraction

Figure 5.10: Defining the voids in a slice of the upper part (slice 1) of sample 1.

Figure 5.11 shows two domains: the green area, where the flow is free, is modeled as the Stokes region $\Omega_{S}$ and the yellow area, representing the porous medium with a defined permeability $k_{p m}$ is modeled as the Darcy region $\Omega_{D}$. Figure 5.12 shows the detail of how the mesh is separated into two regions. Thus, the pre-processing of these images consists of following above steps until their discretization in a finite elements mesh. 


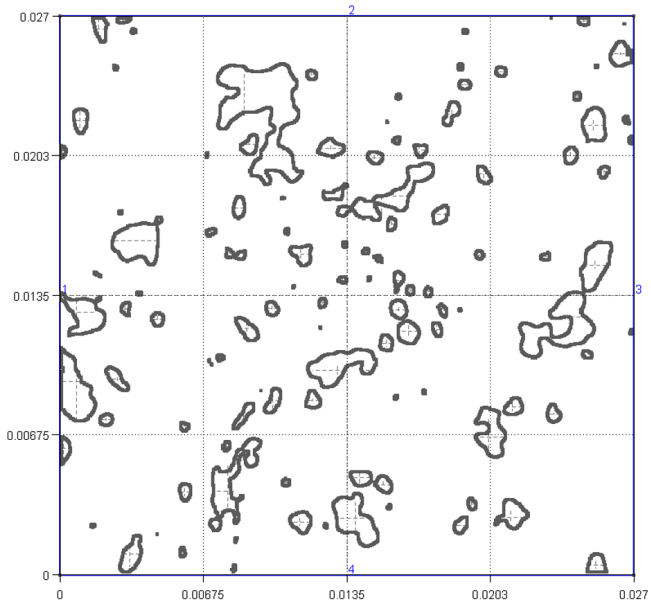

(a) Geometry

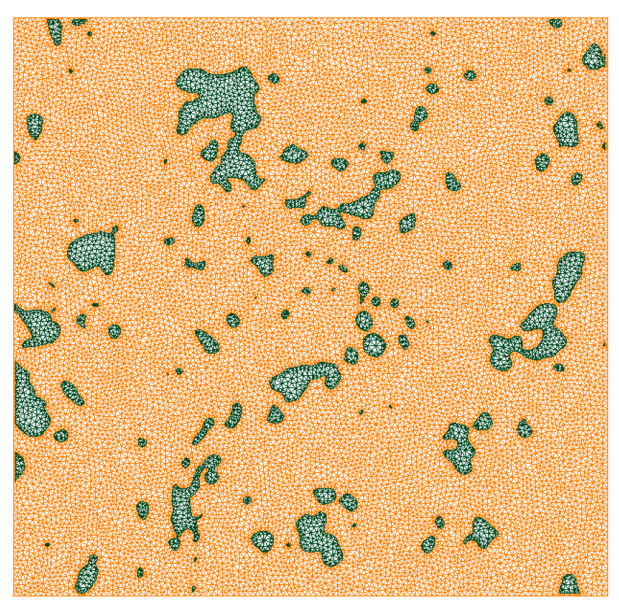

(b) Mesh

Figure 5.11: Domains for one 2D slice of sample 1.

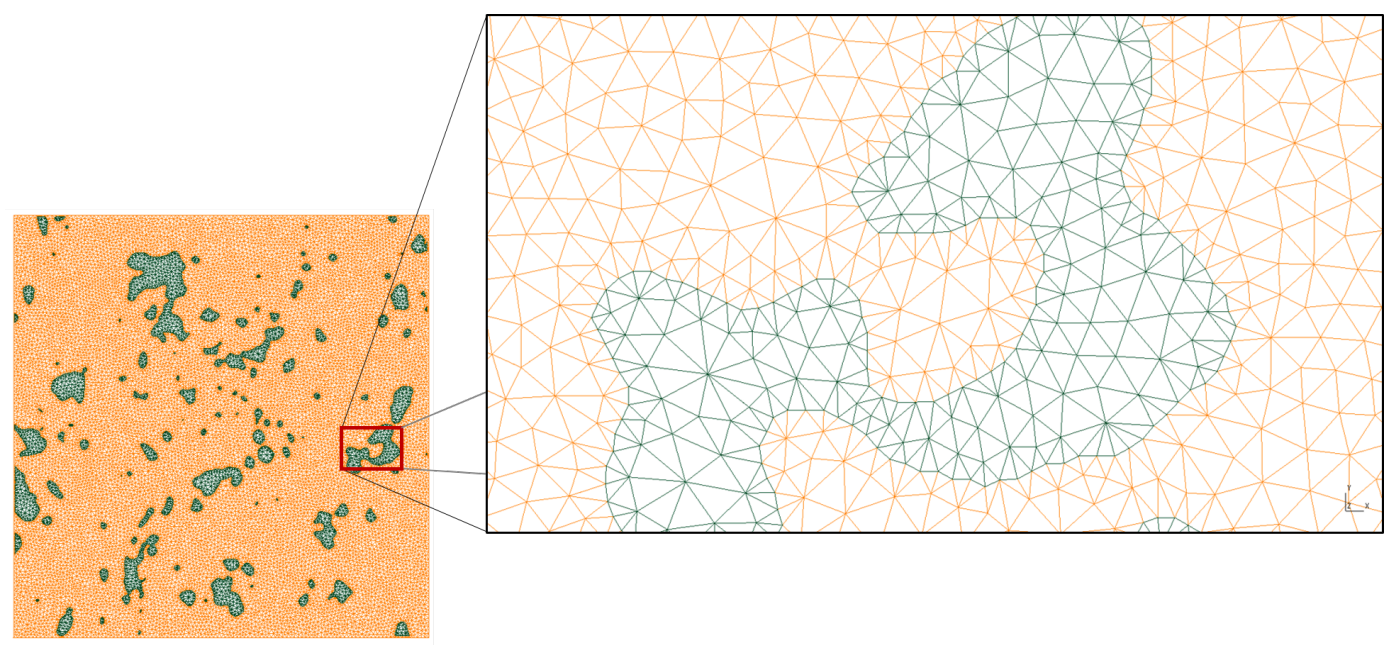

Figure 5.12: Zoom in one part of the mesh to show the limit between the 2 domains.

With the mesh in hand, the code developed in Python to solve Brinkman's model is used to numerically solve the horizontal laminar flow of a fluid through the $2 \mathrm{D}$ domain shown in fig. 5.13. The geometry boundary was divided into 4 segments corresponding to the boundaries at left, top, right and bottom of the domain. In these computations, a pressure gradient was imposed at inlet, left boundary, and outlet of the geometry, right boundary. Impermeable condition is applied in the top and bottom boundaries and a scalar permeability was defined for the porous material. The liquid considered in this flow has demineralized water properties.

The hypothesis considered in this part was the same for the mathematical model, which are steady-state regime, fully-developed laminar flow in the inlet 
and outlet planes, rigid porous matrix, Newtonian and incompressible liquid, isothermal flow, no chemical interaction, neglected gravitational and inertial effects.

A personal computer with Ubuntu 17.10 64-bit operating system was used in these simulations under an Intel Core i7-7700 3.6 GHz processor and 8 GB of RAM memory. The execution time of the program for a mesh with 122,724 elements was 29.8 seconds and for a core analysis with a mesh of 37,606 elements was 10.98 seconds. The solution is then post-processed to generate pressure and velocity fields plots.

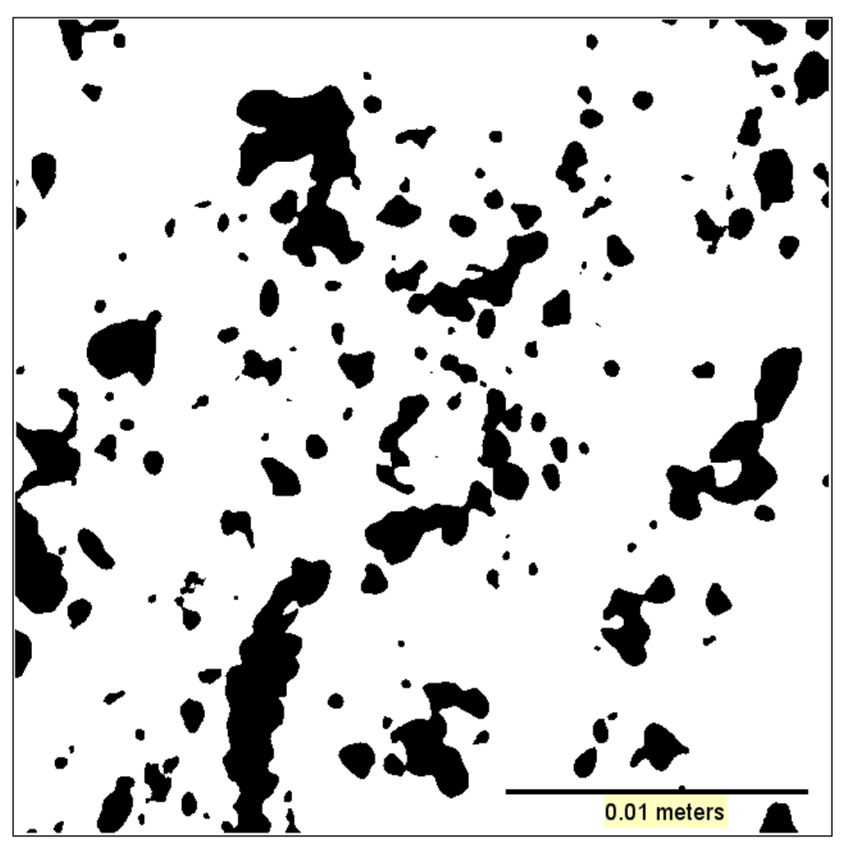

Figure 5.13: Section under investigation.

Using the mesh based in fig. 5.13, it is possible to study the impact of the vug presence in pressure and velocity fields. Figure 5.14 shows the pressure field in the entire domain and along the vertical and horizontal lines marked in fig. 5.14a. It is important to note that the pressure is almost constant inside each vug.

Figure 5.15 shows the velocity vectors, colored by their magnitude. Clearly, the liquid velocity is much higher inside the vugs, where the flow resistance is smaller. Figures 5.16 and 5.17 show the horizontal and vertical velocity along a vertical and horizontal line in the domain, for the conditions of fig. 5.15 . 

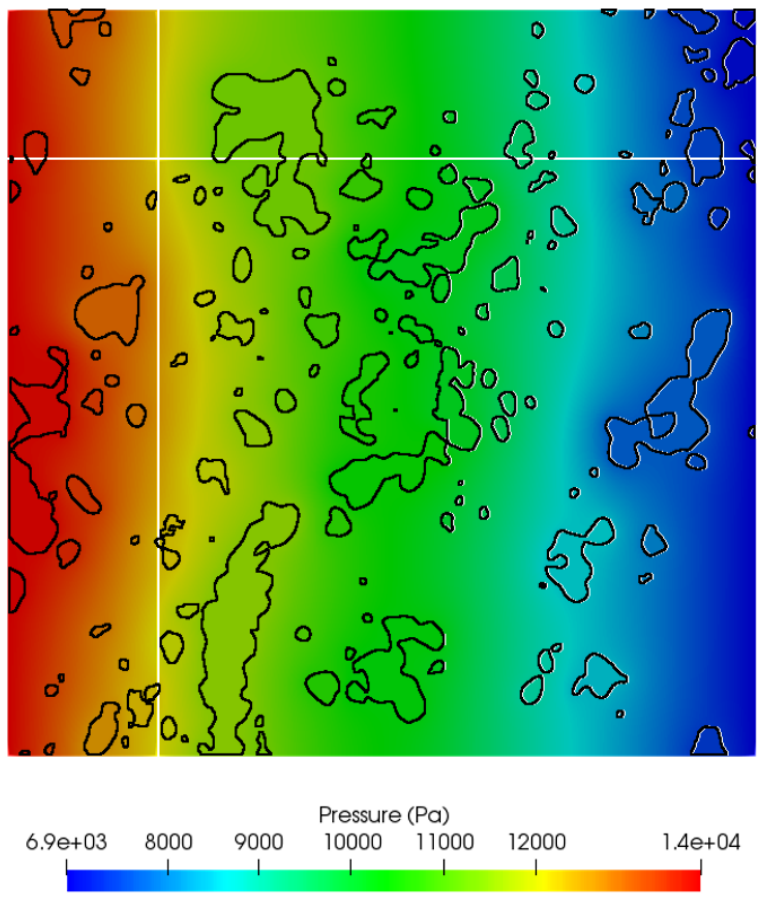

(a) Pressure field.
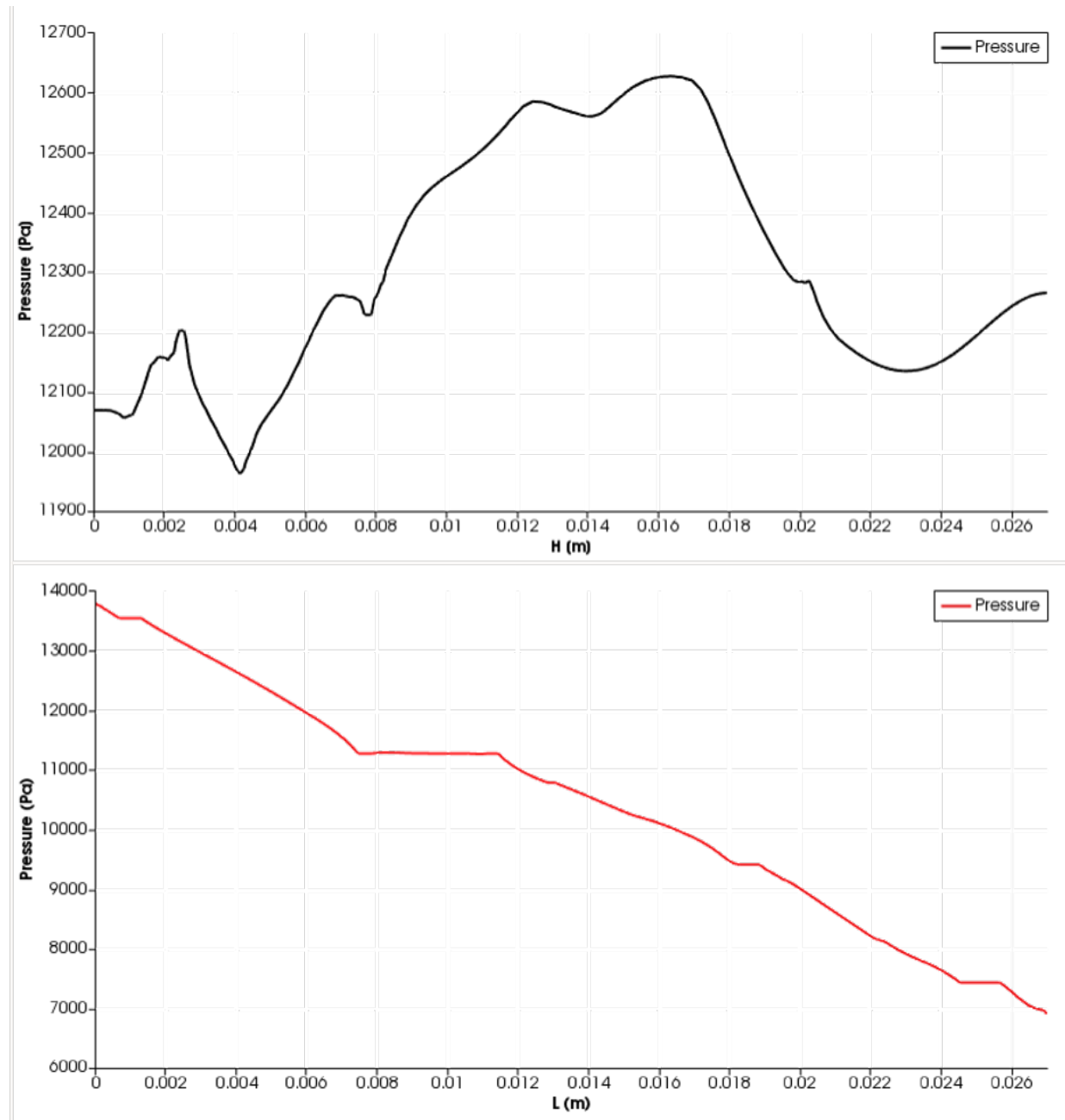

(b) Pressure along $x$ and $y$ lines.

Figure 5.14: Pressure field: $\mathrm{x}$-axis and y-axis with fluid flowing in the $\mathrm{x}$ direction, $\Delta P=6,894.76 \mathrm{~Pa}$ and $k_{p m}=10^{-12} \mathrm{~m}^{2}$. 


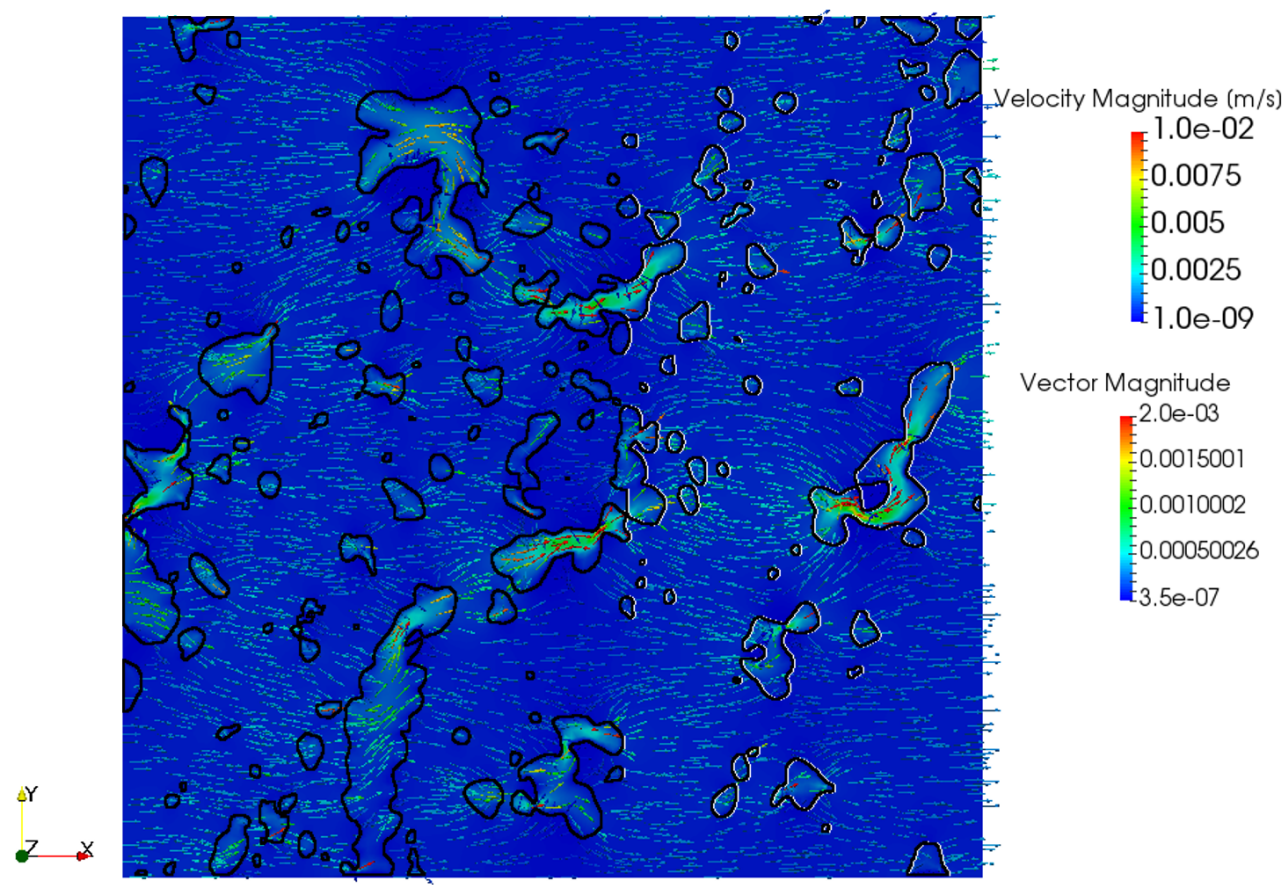

Figure 5.15: Velocity field with fluid flowing in the x-direction, $\Delta P=6,894.76$ $\mathrm{Pa}$ and $k_{p m}=10^{-12} \mathrm{~m}^{2}$.

With the velocity values in hand, the flow rate at the outlet is evaluated for five pressure inputs, $\Delta P=6,894.76,34,473.80,68,947.60,172,369$ and $344,738 \mathrm{~Pa}$, as shown in fig. 5.18.

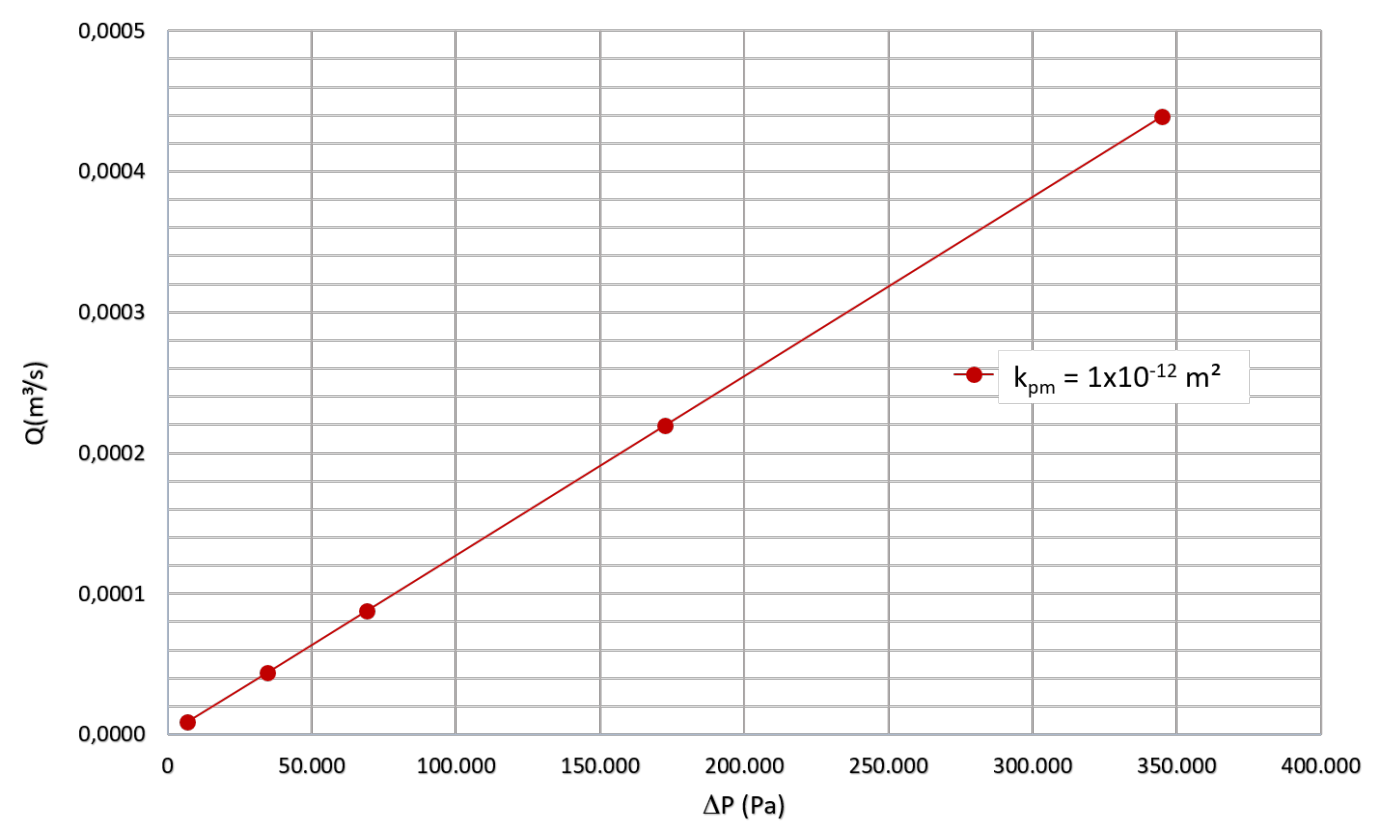

Figure 5.18: Flow rate for different pressure gradient inputs for the domain shown in fig. 5.13 with $k_{p m}=10^{-12} \mathrm{~m}^{2}$ and fluid flowing in the $x$ direction.

For further analysis, it is necessary to investigate if the results are influenced by the flow direction. So, in a similar way to the previous one, 


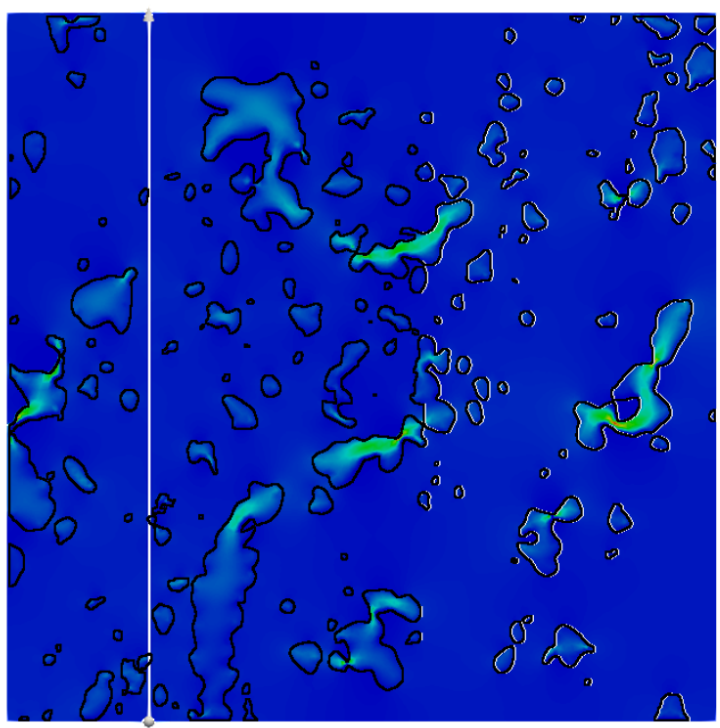

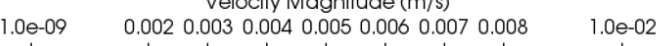
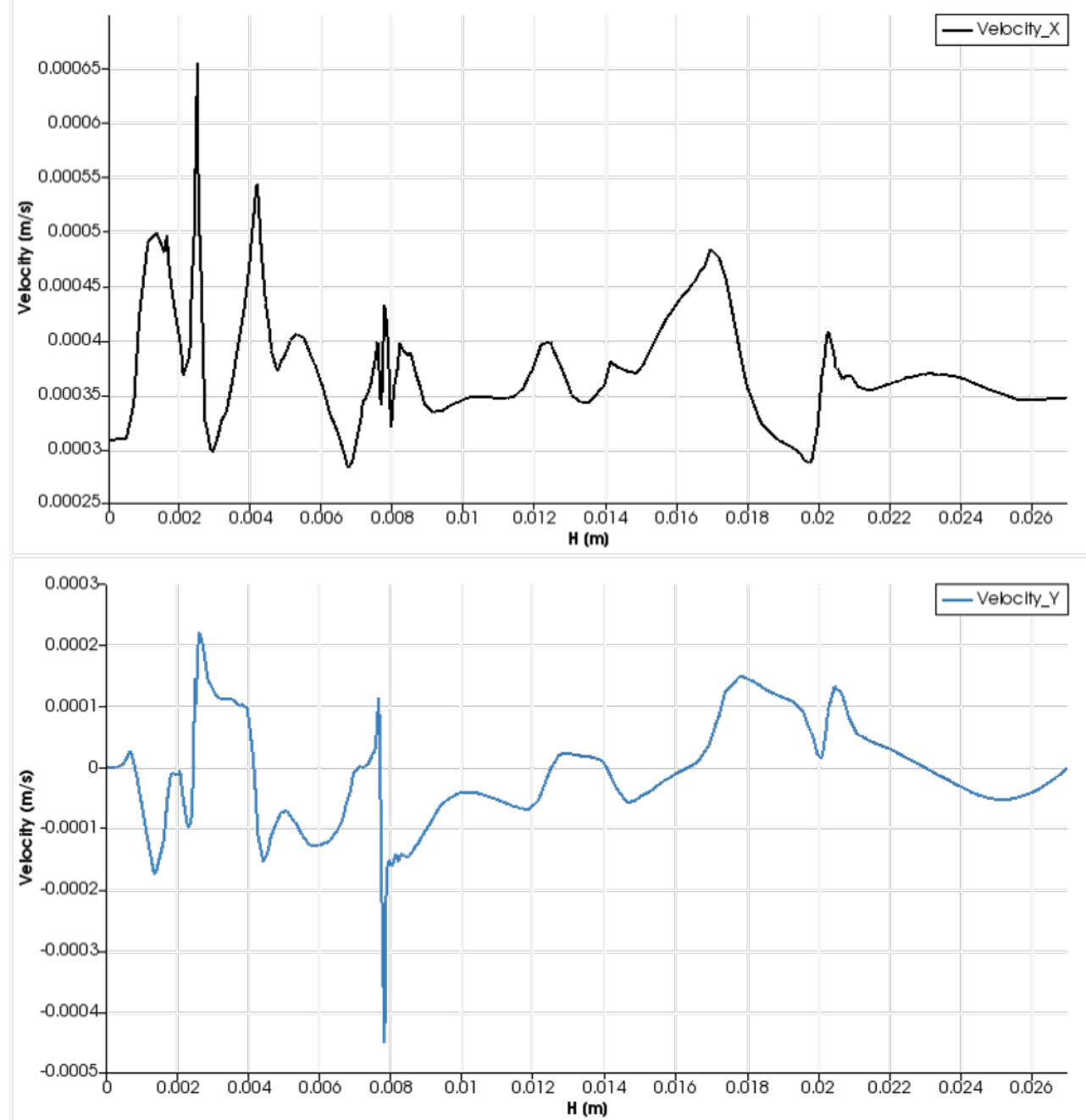

Figure 5.16: Velocity field: y-axis with fluid flowing in the x-direction, $\Delta P=$ 6, 894.76 $\mathrm{Pa}$ and $k_{p m}=10^{-12} \mathrm{~m}^{2}$. 


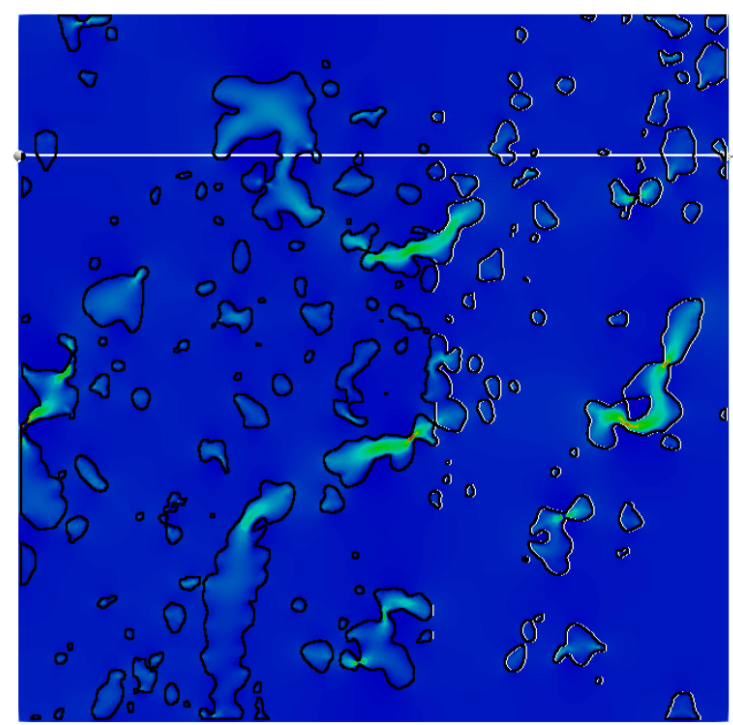

Velocity Magnitude (m/s) $\begin{array}{lllllllllllll}1.0 \mathrm{e}-09 & 0.002 & 0.003 & 0.004 & 0.005 & 0.006 & 0.007 & 0.008 & 1.0 \mathrm{e}-02\end{array}$
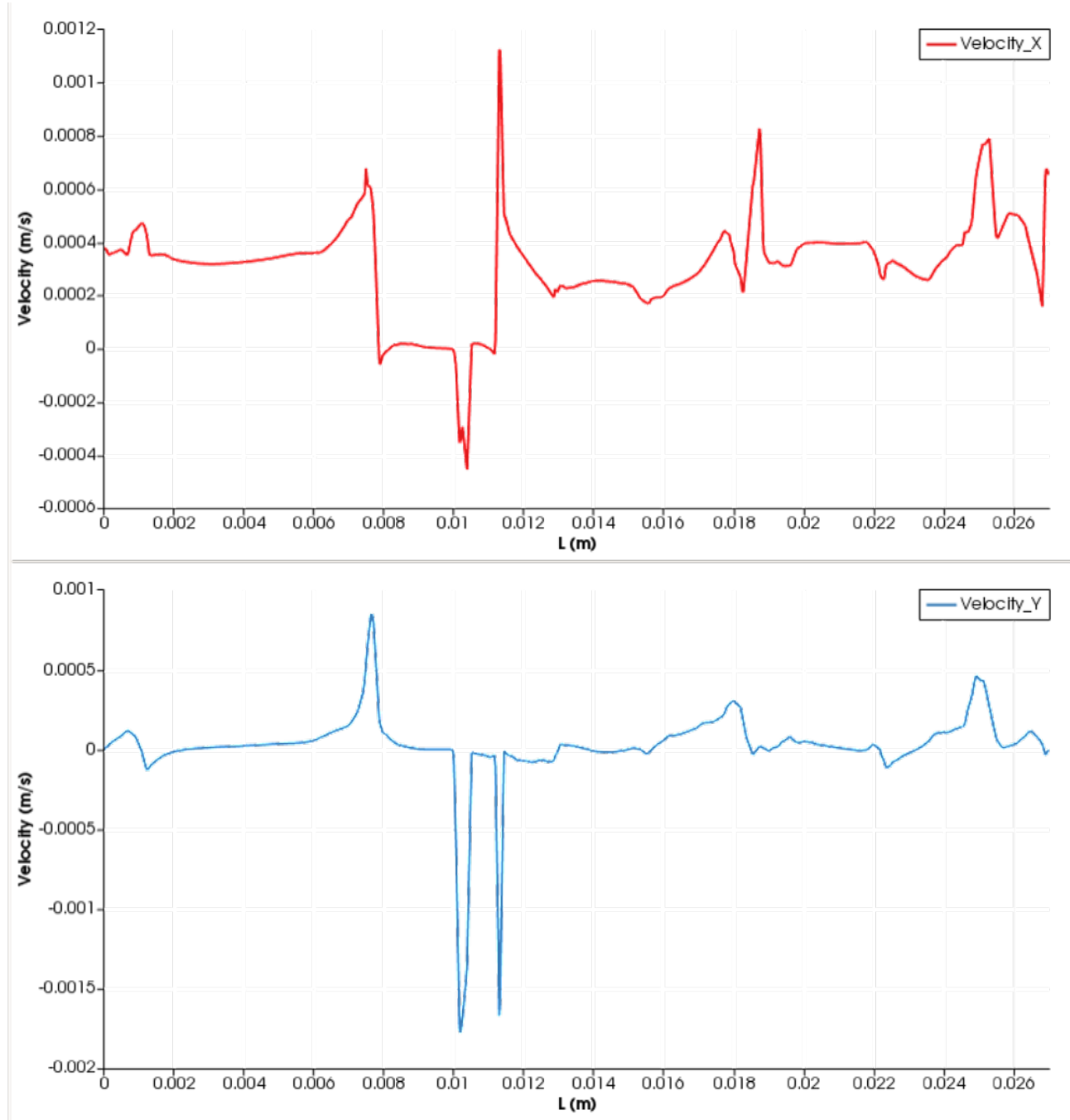

Figure 5.17: Velocity field: $\mathrm{x}$-axis with fluid flowing in the x-direction, $\Delta P=$ 6, 894.76 Pa and $k_{p m}=10^{-12} \mathrm{~m}^{2}$. 
a pressure difference between the bottom and top boundaries was supposed, causing a flow in the vertical direction. The pressure and velocity field are shown in figs. 5.19, 5.20 and 5.21, respectively.

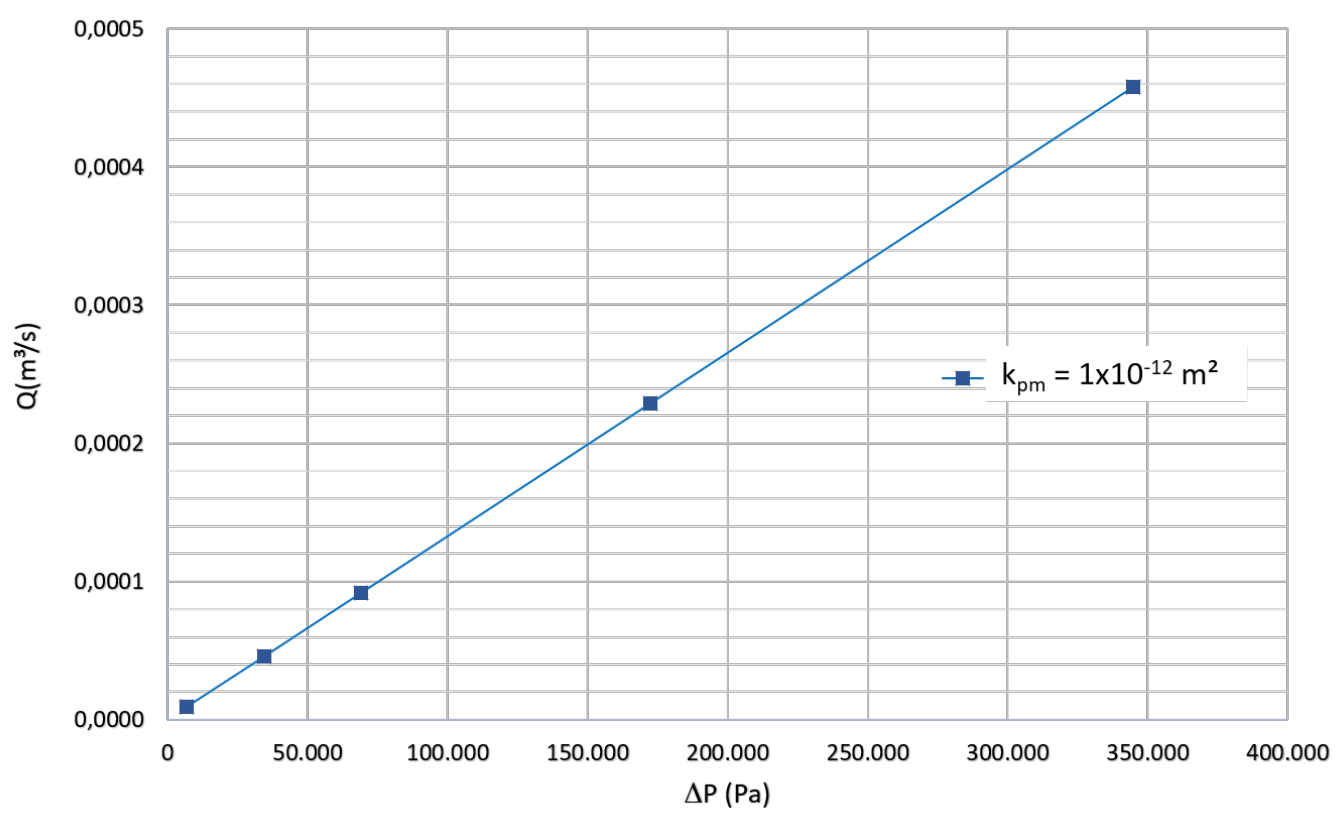

Figure 5.22: Flow rate for different pressure gradient inputs for the domain shown in fig. 5.13 with $k_{p m}=10^{-12} \mathrm{~m}^{2}$ and fluid flowing in the y-direction.

The flow rate in the vertical direction as a function of the imposed pressure difference is shown in fig. 5.22. Figure 5.23 shows a comparison of the flow behavior in both directions. For this domain (geometry and configuration of vugs), the flow rate is slightly higher for the flow in the vertical direction using the same simulation parameters. 

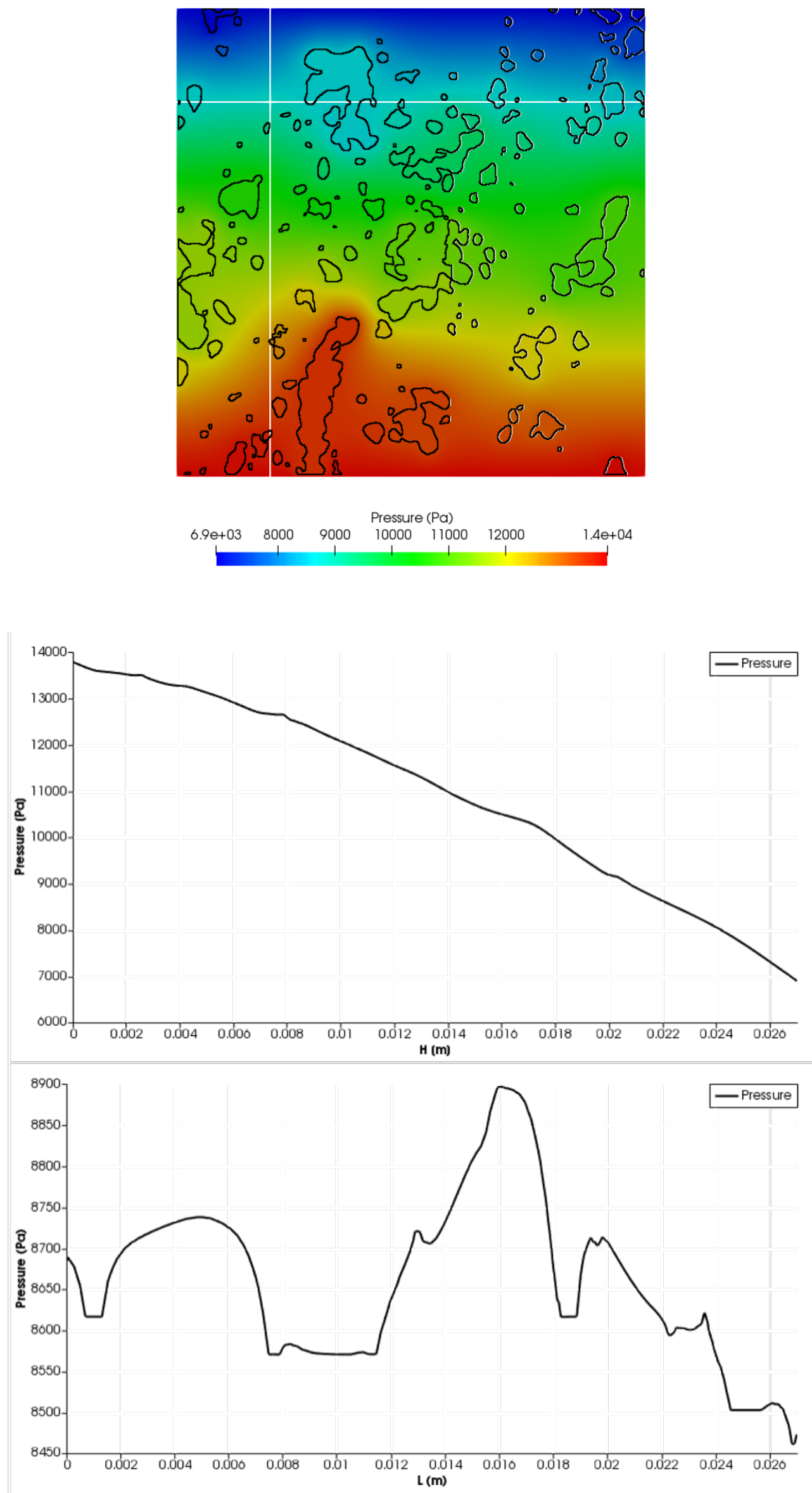

Figure 5.19: Pressure field: $\mathrm{x}$-axis and y-axis with fluid flowing in the $\mathrm{y}$ direction, $\Delta P=6,894.76 \mathrm{~Pa}$ and $k_{p m}=10^{-12} \mathrm{~m}^{2}$. 


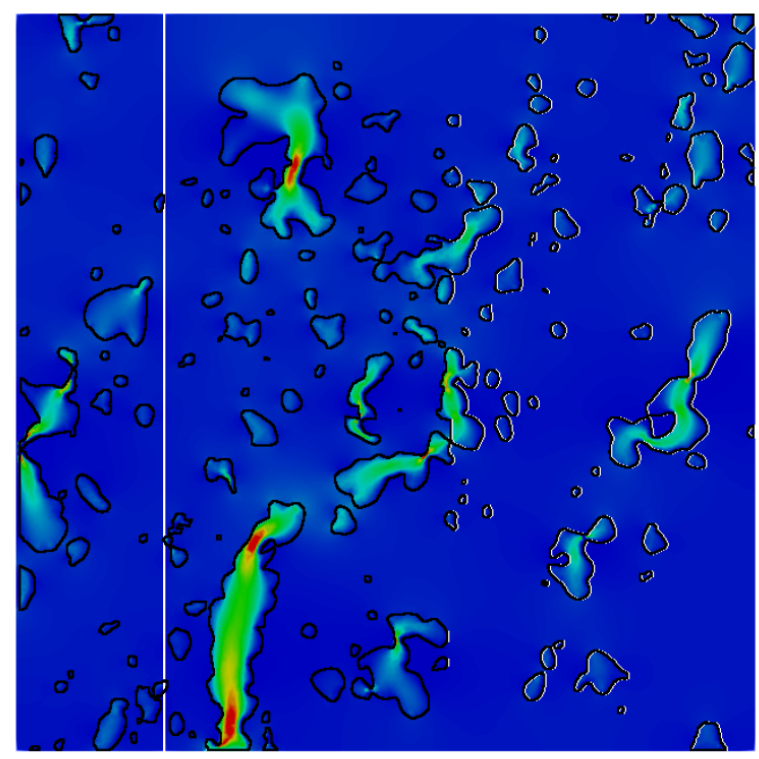

Velocity Magnitude $(\mathrm{m} / \mathrm{s})$

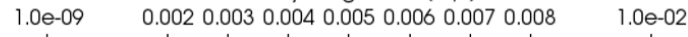
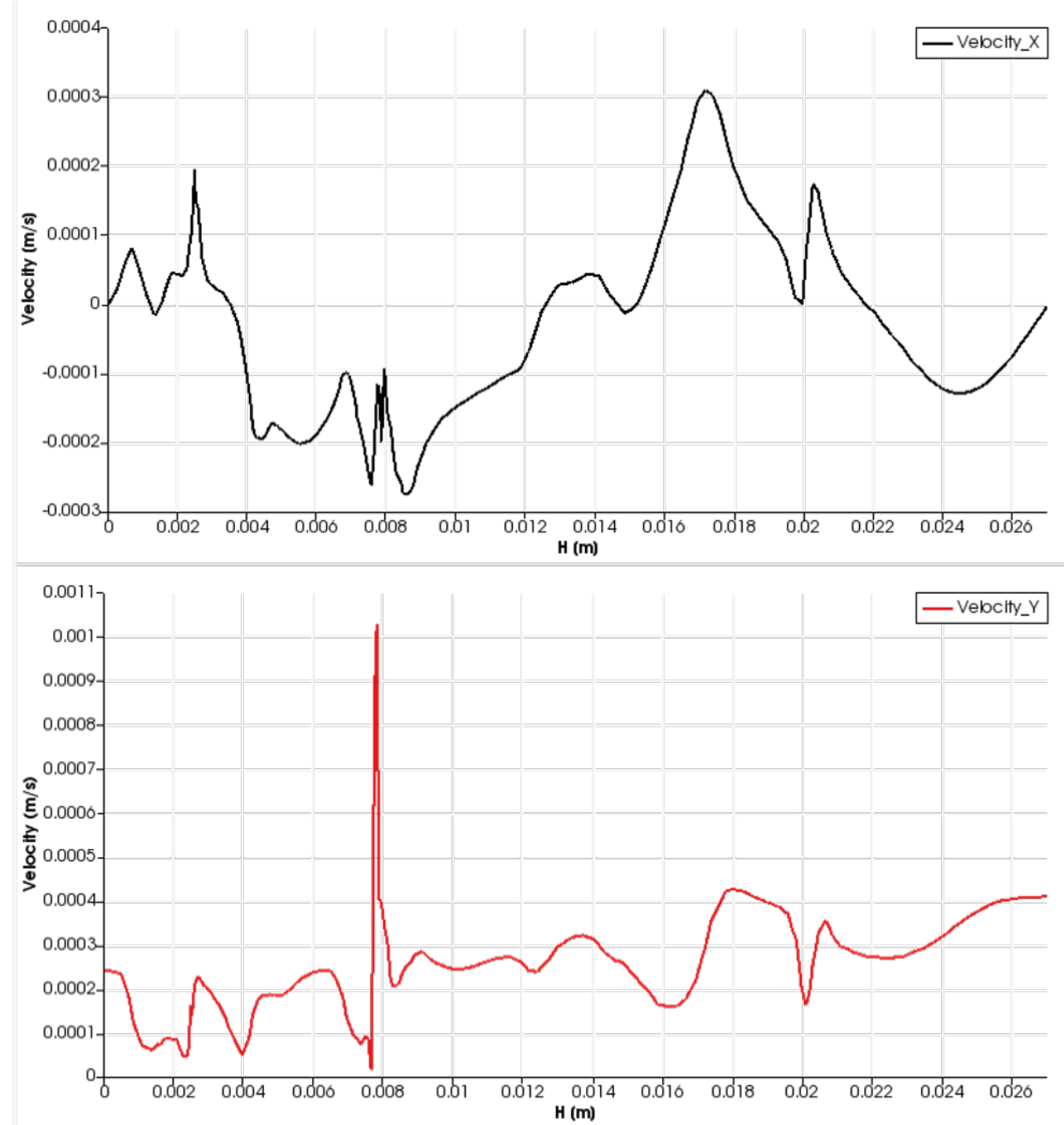

Figure 5.20: Velocity field: y-axis with fluid flowing in the y-direction, $\Delta P=$ $6,894.76 \mathrm{~Pa}$ and $k_{p m}=10^{-12} \mathrm{~m}^{2}$. 

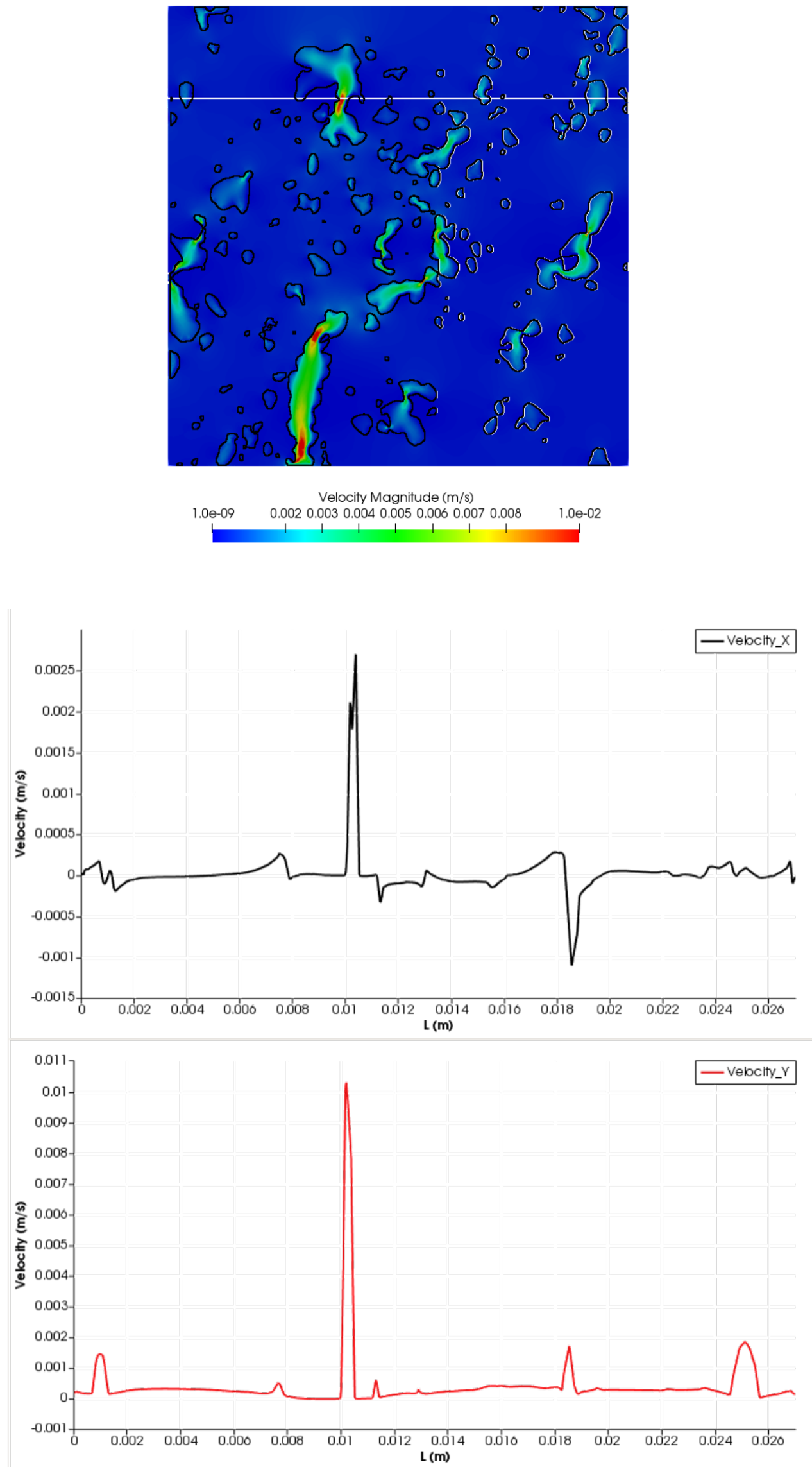

Figure 5.21: Velocity field: $\mathrm{x}$-axis with fluid flowing in the y-direction, $\Delta P=$ $6,894.76 \mathrm{~Pa}$ and $k_{p m}=10^{-12} \mathrm{~m}^{2}$. 


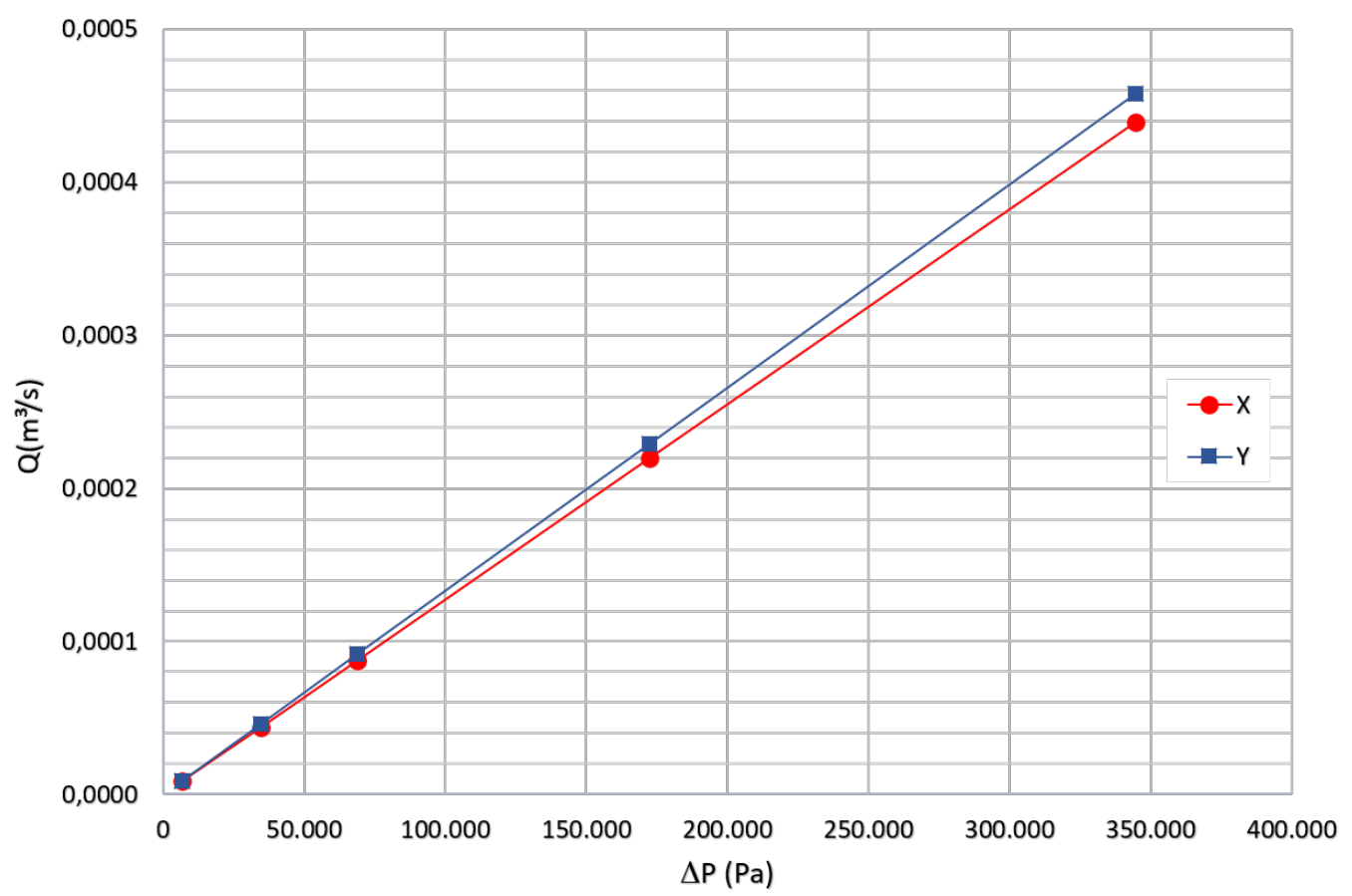

Figure 5.23: Comparison of the flow rate as function of pressure gradient on both flow directions with $k_{p m}=10^{-12} \mathrm{~m}^{2}$ for the domain shown in 5.13.

With the analysis of the graphs shown, it was observed the change in flow pattern near vugs, as high velocities and uniform pressure inside the voids, and the pressure distribution along the section respecting the imposed pressure values at inlet and outlet boundaries. Somehow the vugs arrangement in the investigated domain favored the flow in y-direction resulting in a higher flow rate. In the next sections, the impact of vug shape and flow direction are examined, aiming to identify a particular flow behavior related to vug size.

\subsection{2}

\section{The effect of vug size}

To understand the effect of the size of the cavities on the flow, the methodology presented in the previous sub-section was used in the calculation of the equivalent permeability for different geometries. For this, three distinct filters were applied to the originally treated image of fig. $5.24 \mathrm{a}$ in order to increase the area of the voids, that is, enlarging the already existing ones. Segmentation methods based in global thresholding were used to create more three images virtual manipulated from the core sample image. The threshold methods Moments, Mean and Huang were respectively applied. These methods are used in segmentation to create four distinct zones of vug area and expand the cases analyzed. The section chosen was an image slice of the microtomography referring to the upper part of sample Am08 (Sample 1). 


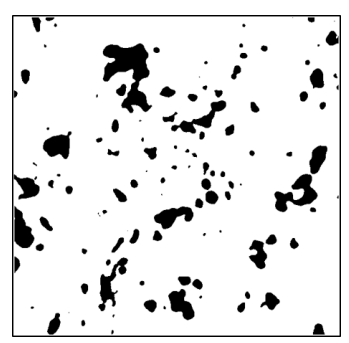

(a) $10.29 \%$ Voids.

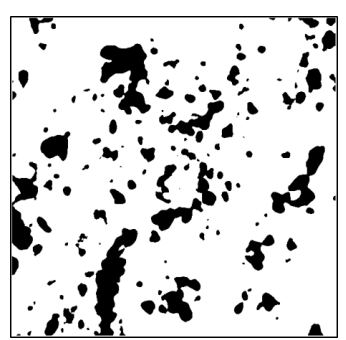

(b) $16.24 \%$ Voids.

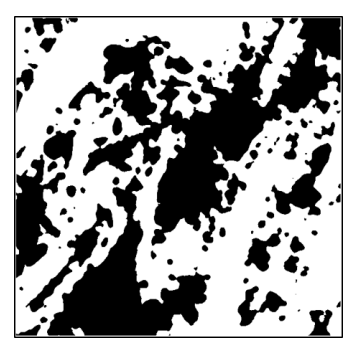

(c) $41.62 \%$ Voids.

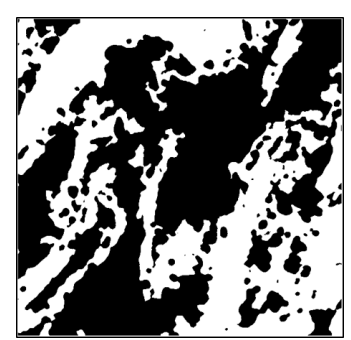

(d) $53.61 \%$ Voids.

Figure 5.24: Vugs enlargement on slice 1 of sample 1. From left to right, the first image is the one which was processed to identify voids as seen in 5.10, next to this were applied different thresholds to enlarge the voids.

Figure 5.24 shows the different geometries considered and the corresponding void porosity $\phi_{\eta}$ (area of the voids over total area of the domain).

The velocity magnitude and pressure field for the four geometries considered are shown in figs. 5.25 and 5.26 for $\Delta P=6,894.76 \mathrm{~Pa}$ and $k_{p m}=10^{-12}$ $\mathrm{m}^{2}$.

For $\phi_{\eta}=10.29 \%$ and $\phi_{\eta}=16.24 \%$, the flow behavior is similar. However, for $\phi_{\eta}>41.6 \%$, there is a strong change in the flow behavior. The very large vugs, led to regions of almost constant pressure and very high velocity. The pressure gradient becomes very strong near the inlet plane. For $\phi_{\eta}=53.6 \%$, the vugs almost percolates from the left to the right boundary, leading to very high velocity through the vugs.

The equivalent permeability of each case is calculated based on the flow predictions as eq. 5-1. The results are presented in fig. 5.27. Tests were also performed for different permeability values of the porous matrix.

$$
k_{e q}=\frac{Q}{A} \cdot \frac{\mu L}{\Delta P}
$$




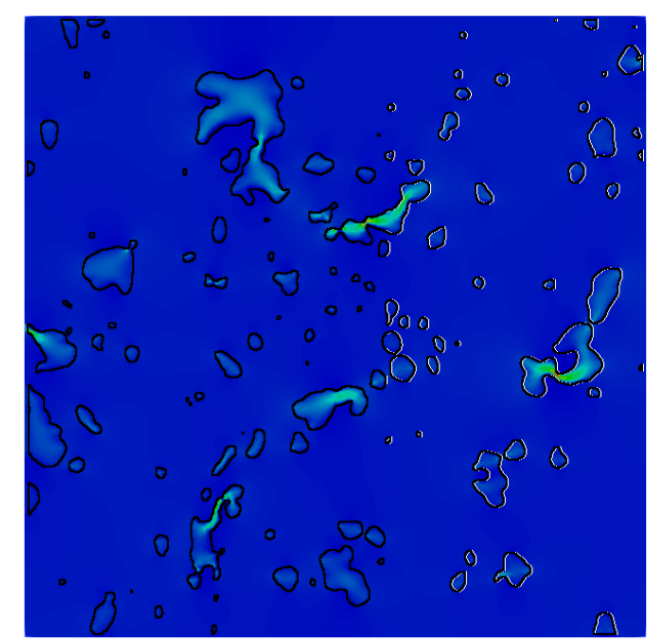

$\begin{array}{lll}\text { Velocity Magnitude }(\mathrm{m} / \mathrm{s}) & \text { Pressure (Pa) }\end{array}$

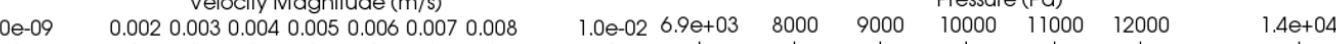

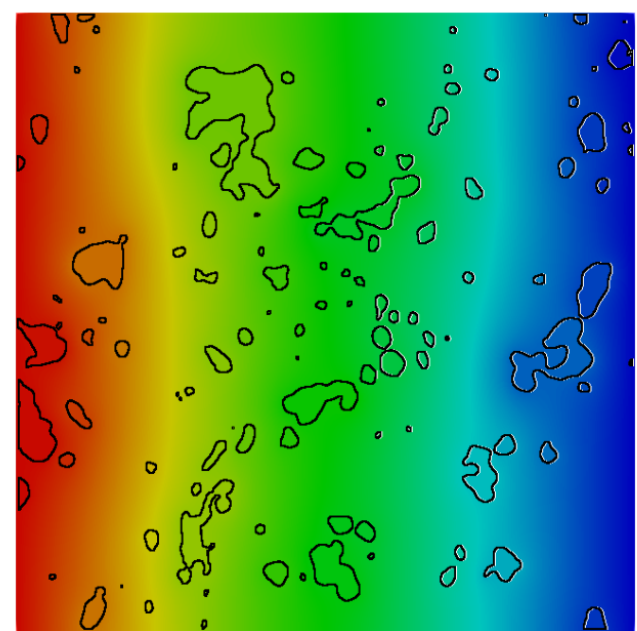

(a) $10.29 \%$ Voids.

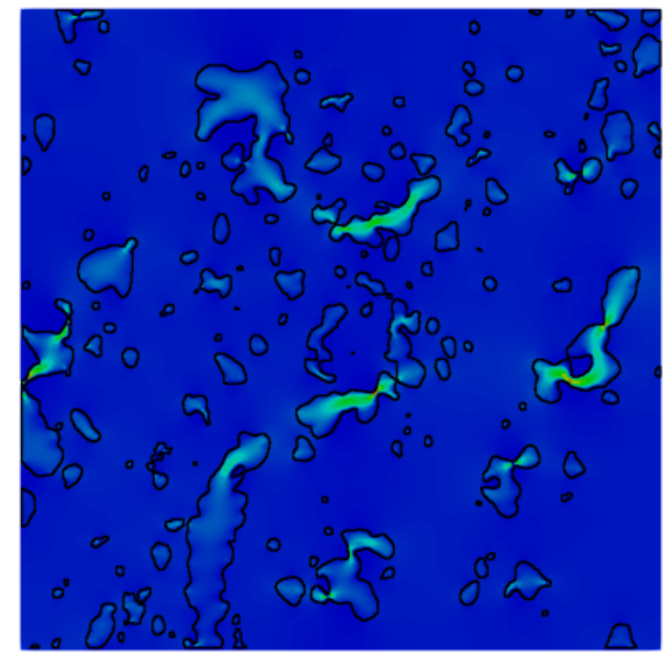

Velocity Magnitude $(\mathrm{m} / \mathrm{s})$

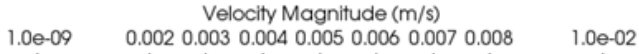

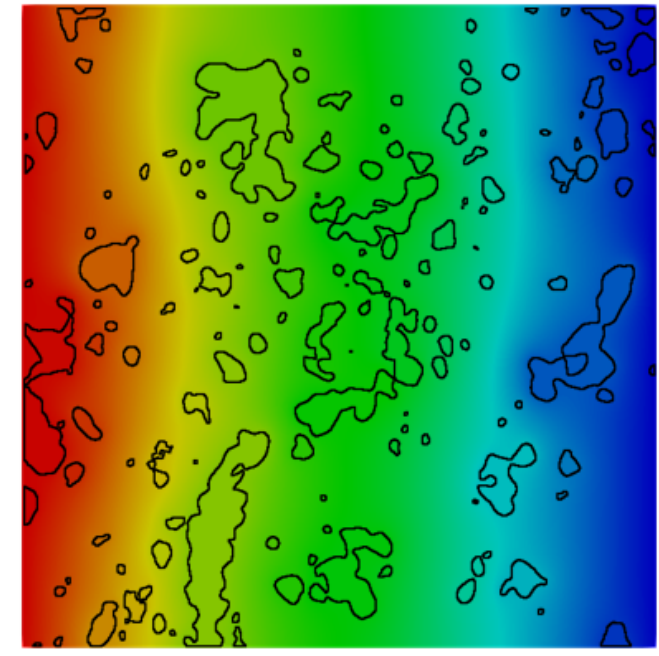

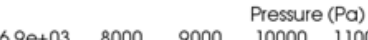

(b) $16.24 \%$ Voids.

Figure 5.25: Pressure and velocity field for first cells described in the domains shown in fig. 5.24 with flow in the x-direction, $\Delta P=6,894.76 \mathrm{~Pa}$ and $k_{p m}=10^{-12} \mathrm{~m}^{2}$. 

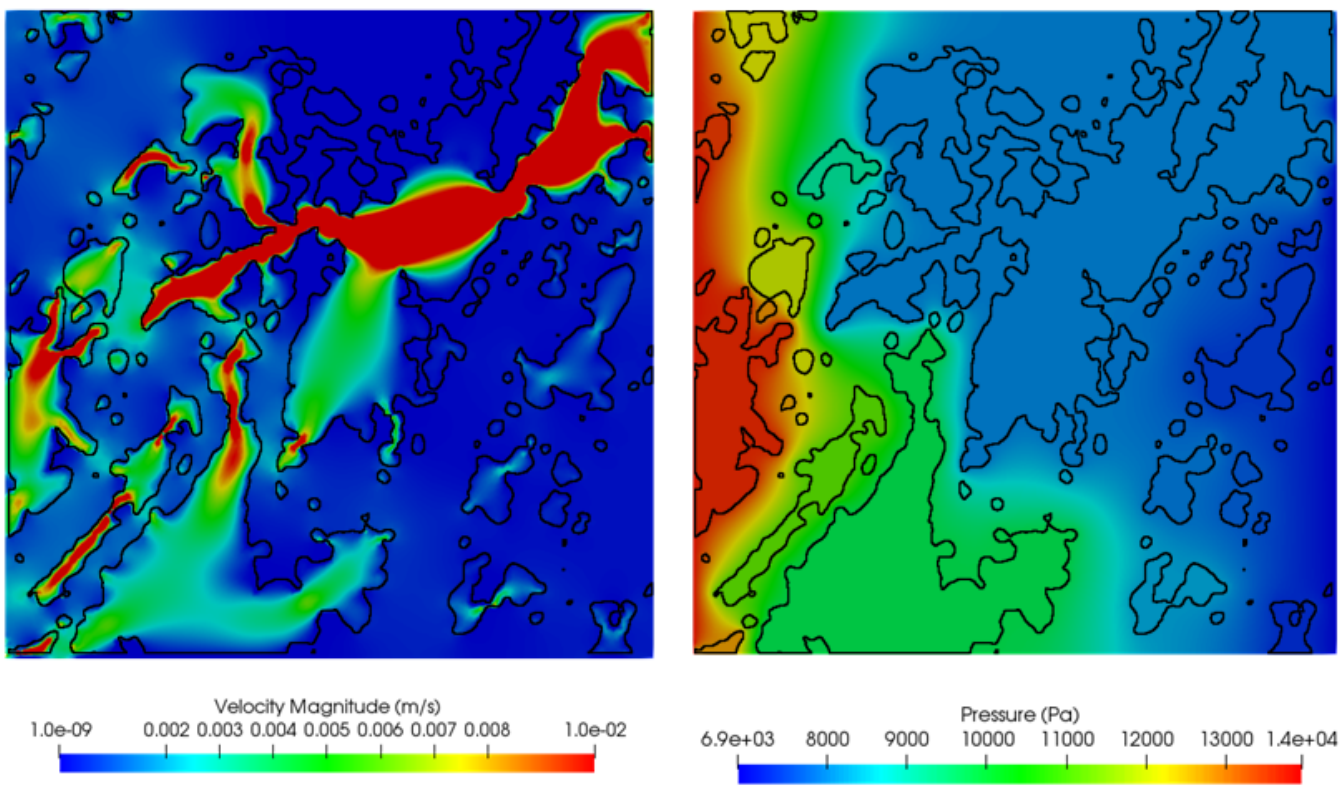

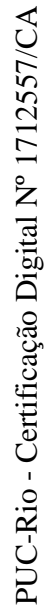

(a) $41.62 \%$ Voids.
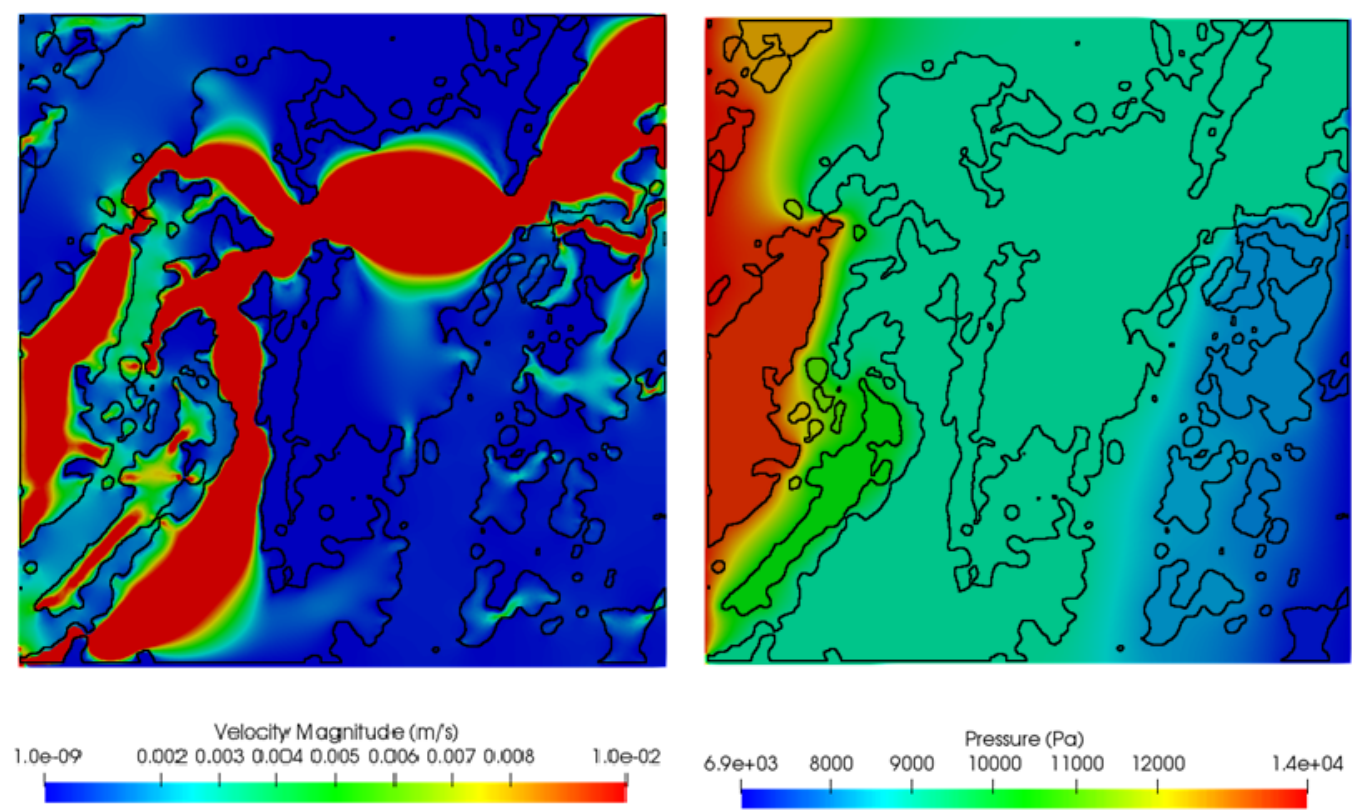

(b) $53.61 \%$ Voids.

Figure 5.26: Pressure and velocity field for last cells described in the domains shown in fig. 5.24 with flow in the x-direction, $\Delta P=6,894.76 \mathrm{~Pa}$ and $k_{p m}=10^{-12} \mathrm{~m}^{2}$. 


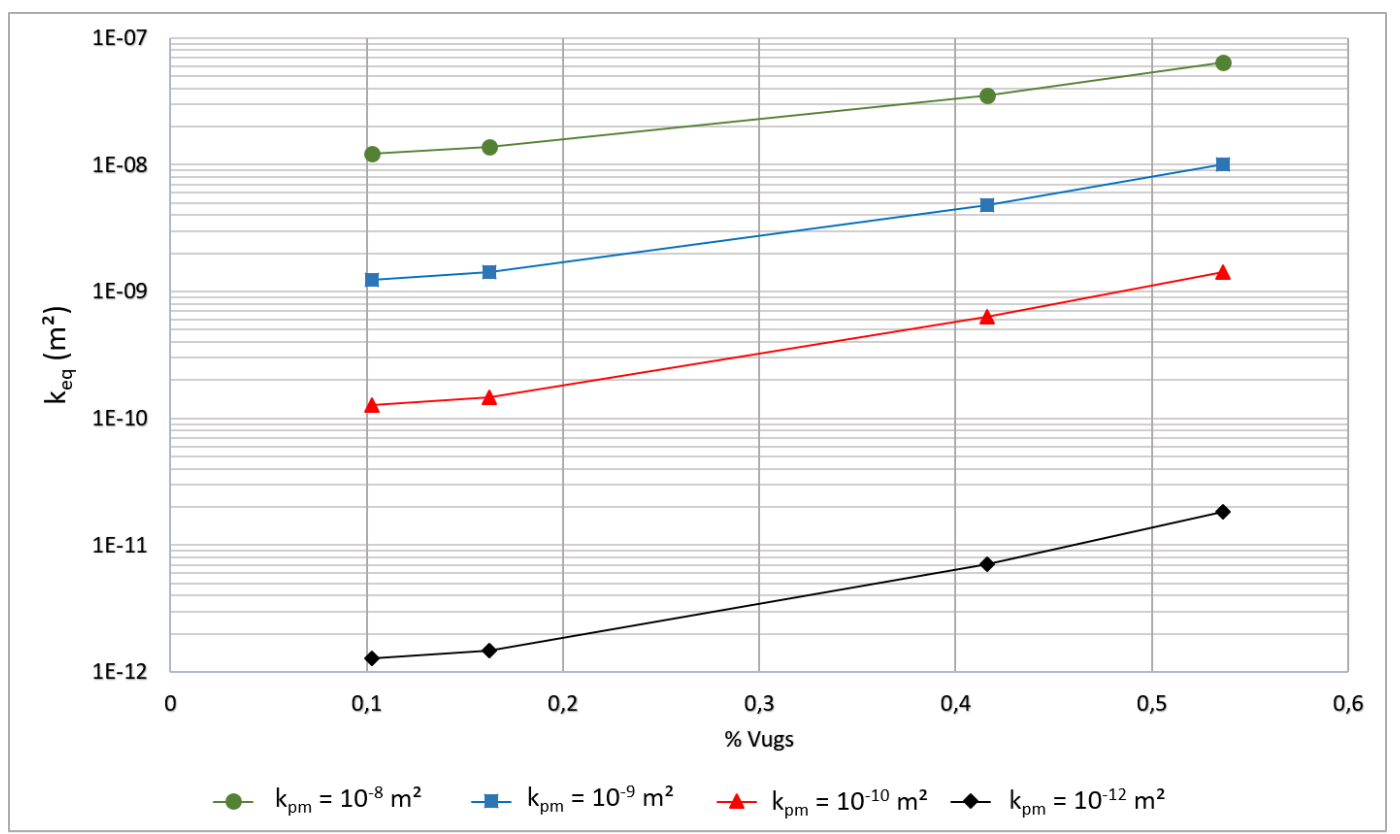

Figure 5.27: Equivalent permeability $\left(k_{e q}\right)$ of the geometries shown in fig. 5.24 with different permeabilities of porous matrix, fluid flowing in the $x$-direction and $\Delta P=6,894.76 \mathrm{~Pa}$.

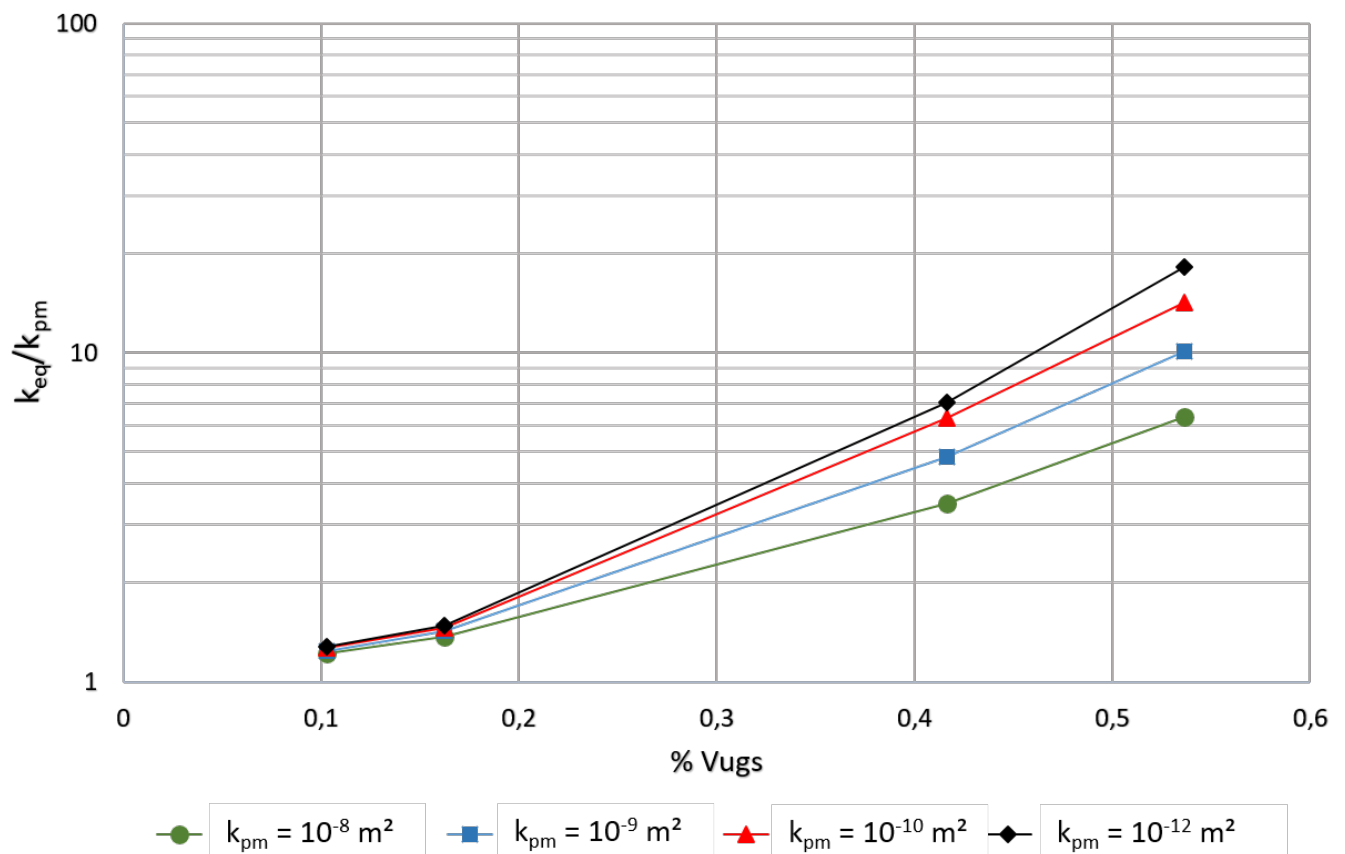

Figure 5.28: Equivalent permeability $\left(k_{e q}\right)$ obtained at the outlet with flow on $x$-direction and $\Delta P=6,894.76 \mathrm{~Pa}$, divided by each porous media permeability $\left(k_{p m}\right)$ as a function of the voids of the cells shown in fig. 5.24. The dependent variable is on the logarithmic scale.

The results are presented as the ratio between the equivalent permeability to the porous matrix permeability in fig. 5.28. For low vug porosity, the ratio is almost independent of the porous matrix permeability. For high vug porosity, 
the $k_{e q} / k_{p m}$ ratio is higher for low $k_{p m}$. The effect of the vugs is stronger in a low permeability porous matrix.

The results for the flow in the vertical direction are shown in figs. 5.29, 5.30 and 5.31. Although the values of the ratio $k_{e q} / k_{p m}$ are different, the conclusions are the same.

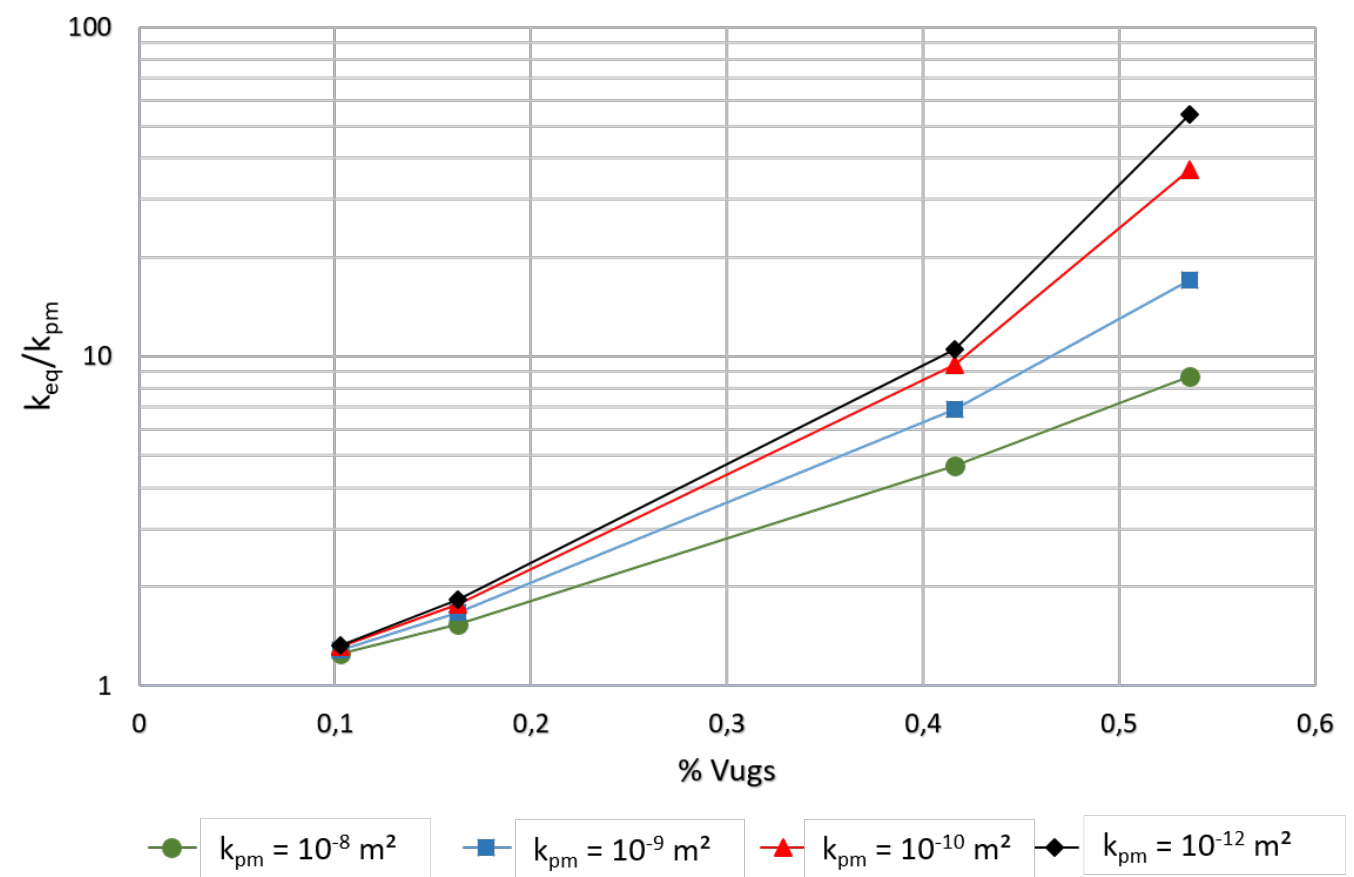

Figure 5.31: Equivalent permeability $\left(k_{e q}\right)$ obtained at the outlet with flow on $y$-direction and $\Delta P=6,894.76 \mathrm{~Pa}$, divided by each porous media permeability $\left(k_{p m}\right)$ as a function of the voids of the cells shown in fig. 5.24. The dependent variable is on the logarithmic scale.

Figure 5.32 compares the equivalent permeability $k_{e q}$ of the flow in the vertical and horizontal direction for $k_{p m}=10^{-12} \mathrm{~m}^{2}$. For low vug porosity, the permeabilities are similar. For high vug porosity, the permeability in the vertical direction flow is much higher. This behavior is associated with the connectivity of the vugs in both directions. 


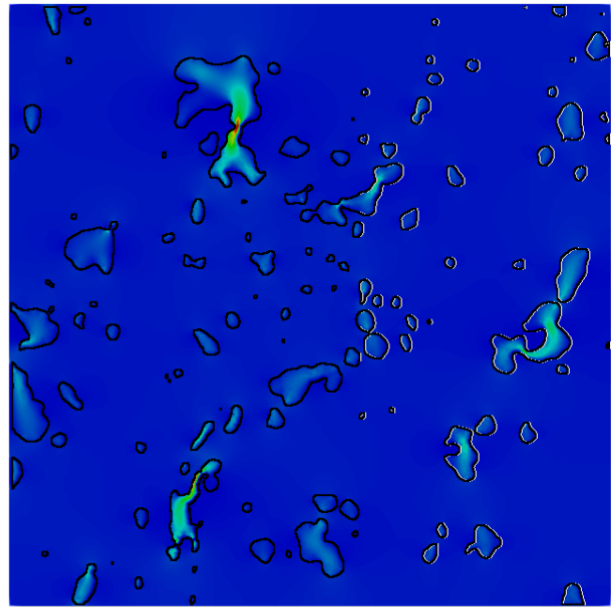

Velocity Magnitude $(\mathrm{m} / \mathrm{s})$

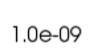

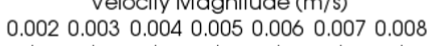

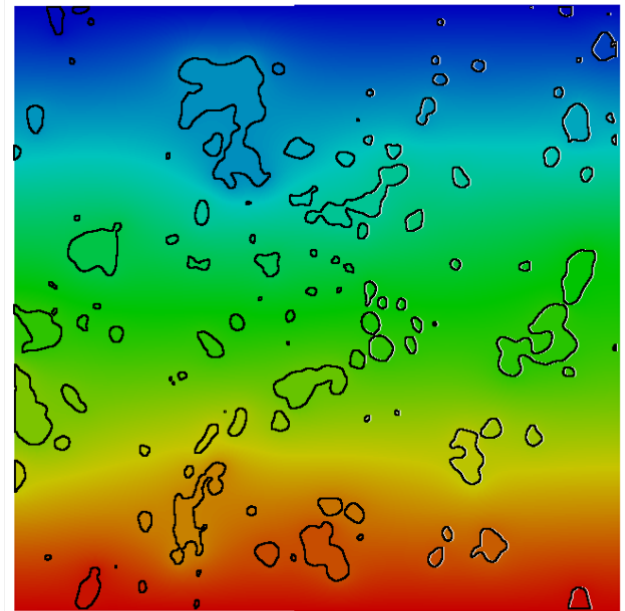

Pressure (Pa)

(a) $10.29 \%$ Voids.
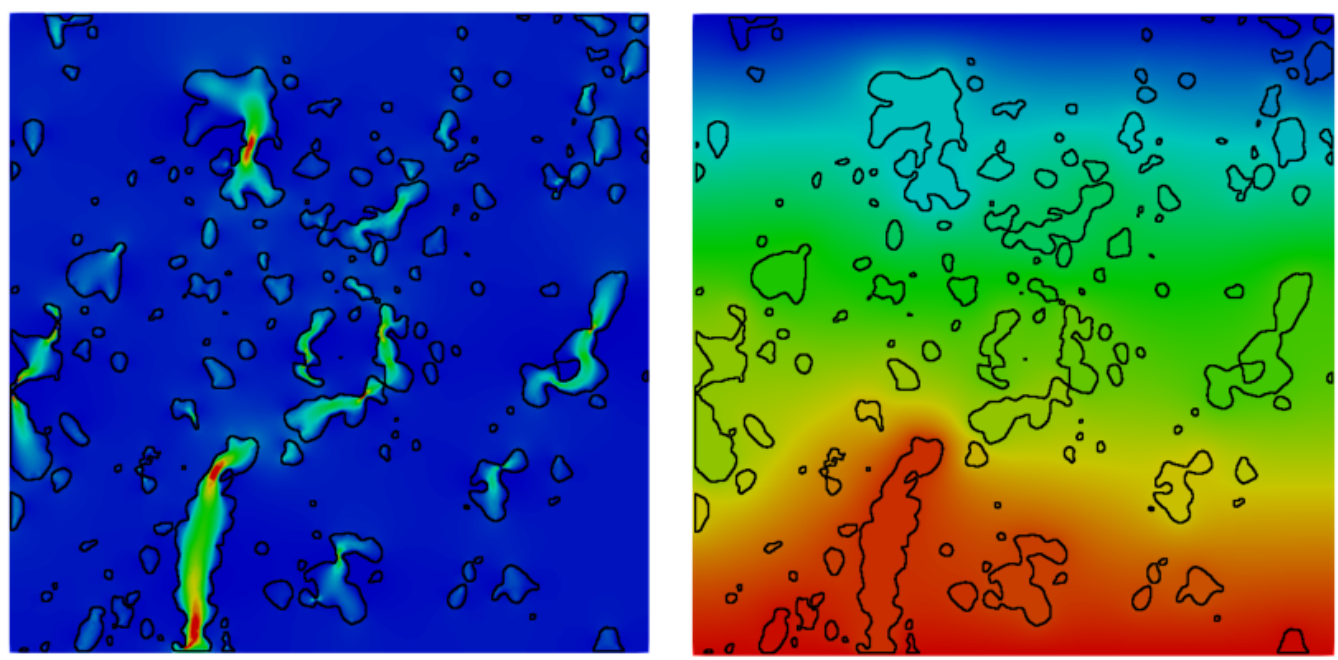

$\begin{array}{cc}\text { Velocity Magnitude }(\mathrm{m} / \mathrm{s}) \\ 1.00-09 & 0.0020 .003 \quad 0.0040 .0050 .0060 .0070 .008\end{array}$

$\begin{array}{llll}1.00-02 & 6.9 e+03 \quad 8000 \quad 9000 & \text { Pressure }(\mathrm{Pa}) \\ & 10000 & 11000\end{array}$

$10000 \quad 11000 \quad 12000 \quad 13000 \quad 1.4 e+04$

(b) $16.24 \%$ Voids.

Figure 5.29: Pressure and velocity field for first cells described in fig. 5.24 with flow in the y-direction, $\Delta P=6,894.76 \mathrm{~Pa}$ and $k_{p m}=10^{-12} \mathrm{~m}^{2}$. 

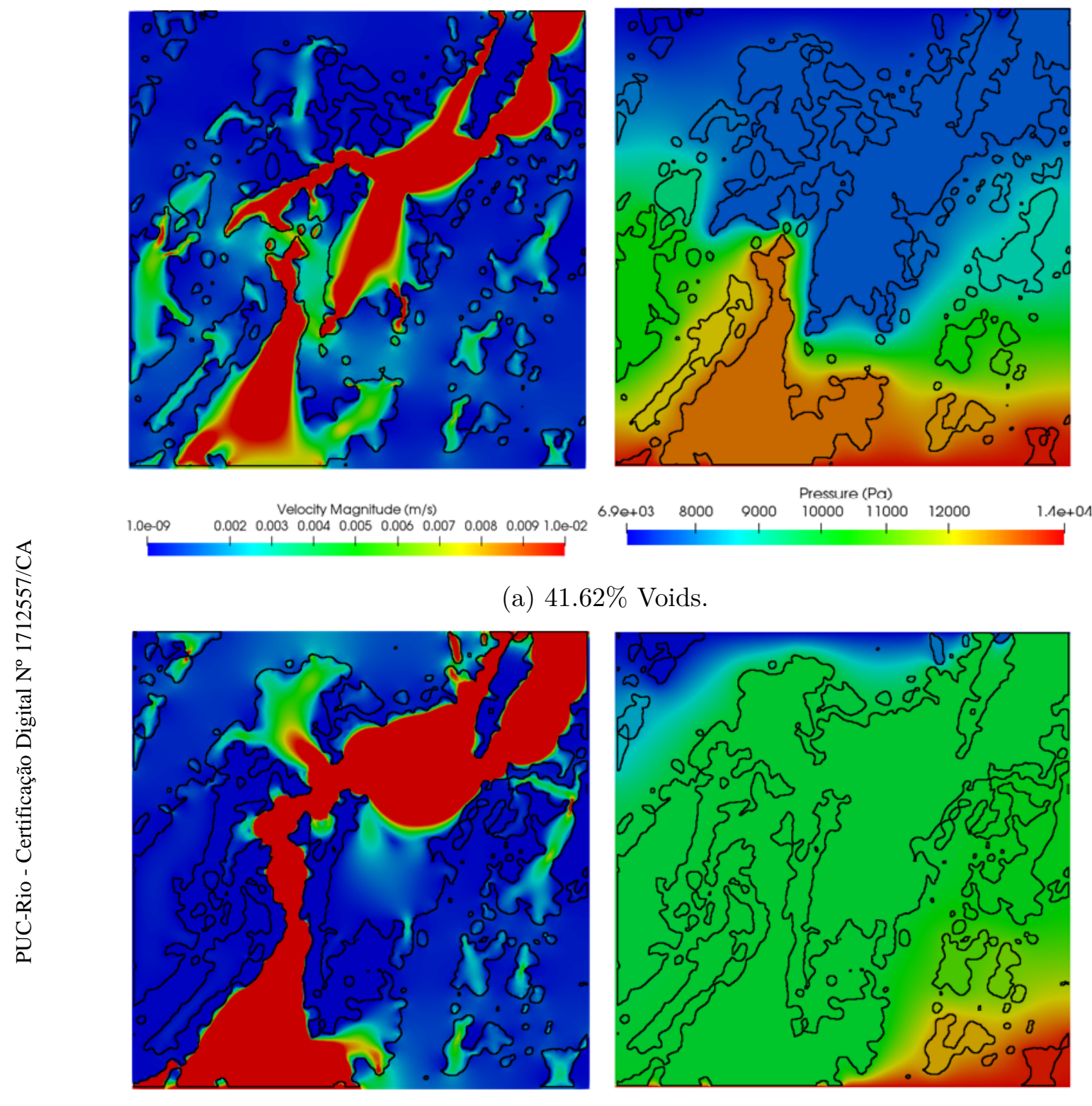

Voids.

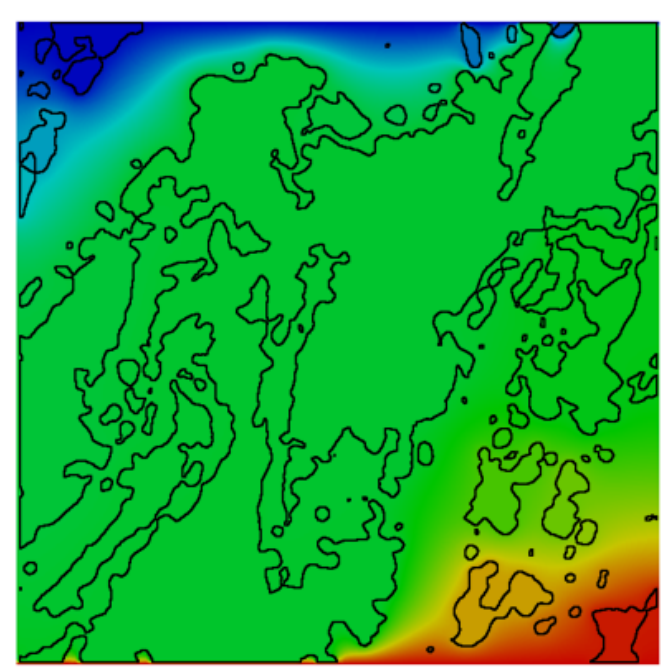

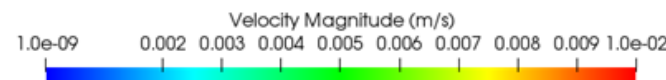

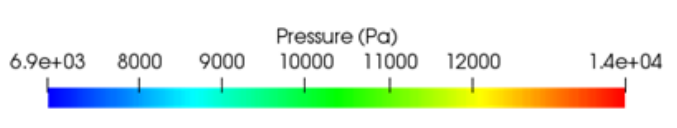

(b) $53.61 \%$ Voids.

Figure 5.30: Pressure and velocity field for last cells described in fig. 5.24 with flow in the y-direction, $\Delta P=6,894.76 \mathrm{~Pa}$ and $k_{p m}=10^{-12} \mathrm{~m}^{2}$. 


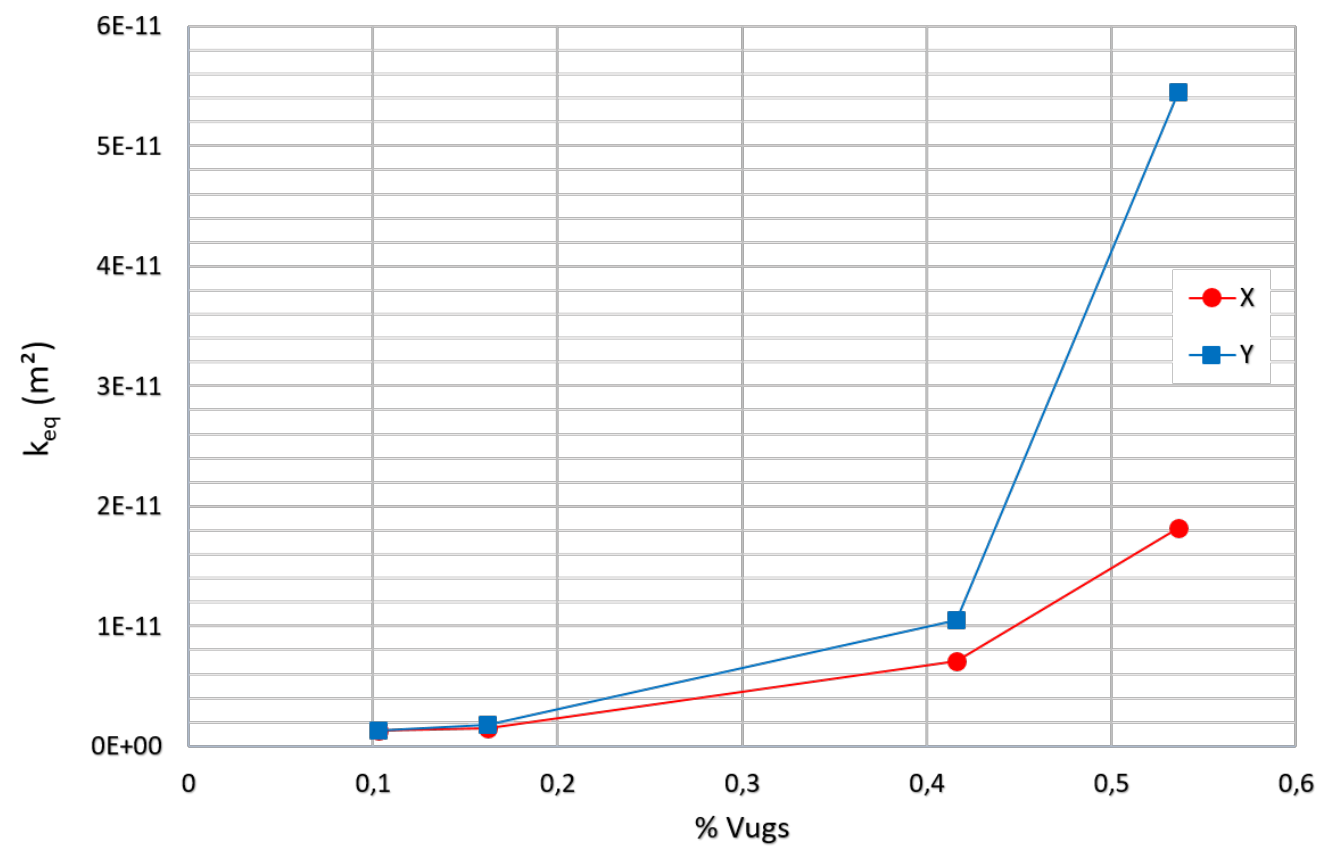

Figure 5.32: Comparison of equivalent permeability as function of vugs area on both flow directions with $k_{p m}=10^{-12} \mathrm{~m}^{2}$ and $\Delta P=6,894.76 \mathrm{~Pa}$ for slice 1 of sample 1.

Other slices of the two samples of carbonates available were analyzed (AM05 and AM08). Three slices were selected from each sample, one from the upper, one from the middle and one from the bottom sections. Then, during the treatment of images, three filters to enlarge the black areas (vugs) were applied in order to study the effect of vug porosity and connectivity in the equivalent permeability.

In the left-hand column of fig. 5.33 are the images originally processed from the middle and lower parts of sample Am08 for the voids to be identified as done in fig. 5.10. Eroding filters were used to generate images of different vug porosity.

Flow fluids were obtained for both flow in the $x$ and $y$ directions, for an imposed pressure gradient of $6,894.76 \mathrm{~Pa}$ and a range of porous matrix permeabilities $\left(k_{p m}=10^{-12} \mathrm{~m}^{2}\right.$ to $\left.10^{-8} \mathrm{~m}^{2}\right)$. The equivalent permeability in units of the porous matrix permeability for all the images of figs. 5.24 and 5.33, and both flow directions are presented in fig. 5.34. 


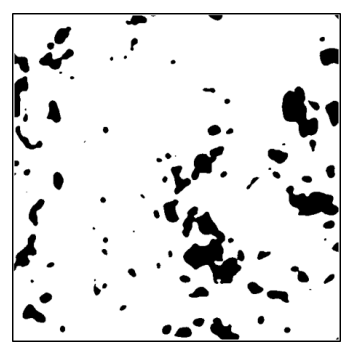

(a) $10.67 \%$ Voids.

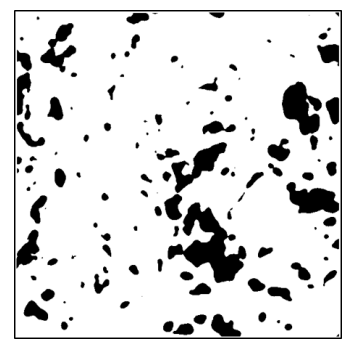

(b) $14.47 \%$ Voids.

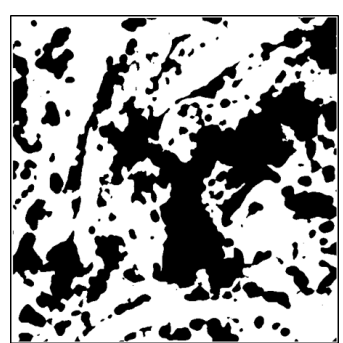

(c) $39.45 \%$ Voids.

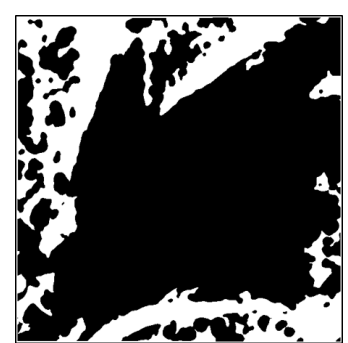

(d) $72.52 \%$ Voids.

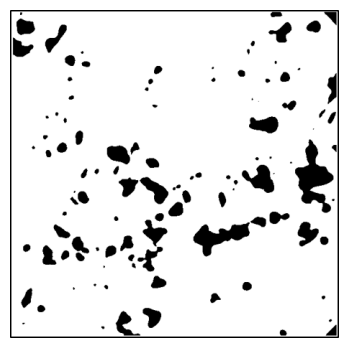

(e) $9.36 \%$ Voids.

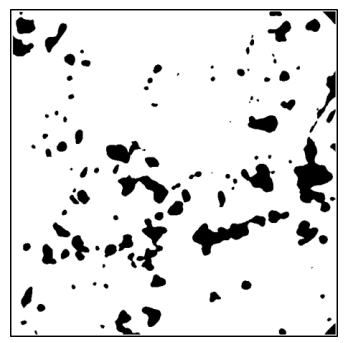

(f) $10.69 \%$ Voids.

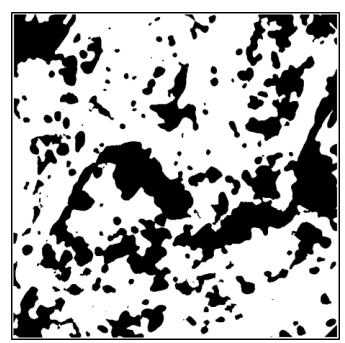

(g) $32.76 \%$ Voids.

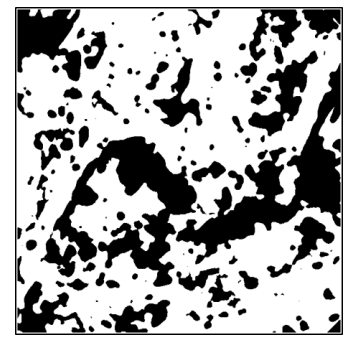

(h) $34.84 \%$ Voids.

Figure 5.33: From b-d are the vugs enlarged of slice 2 (middle part) and from $\mathrm{f}$-h, slice 3 (lower part) of sample 1 . On left column are the ones which were processed to identify voids as done from $5.24 \mathrm{a}$ to $5.24 \mathrm{~b}$, next to this were applied different thresholds to enlarge voids with same threshold methods applied in fig. 5.24.

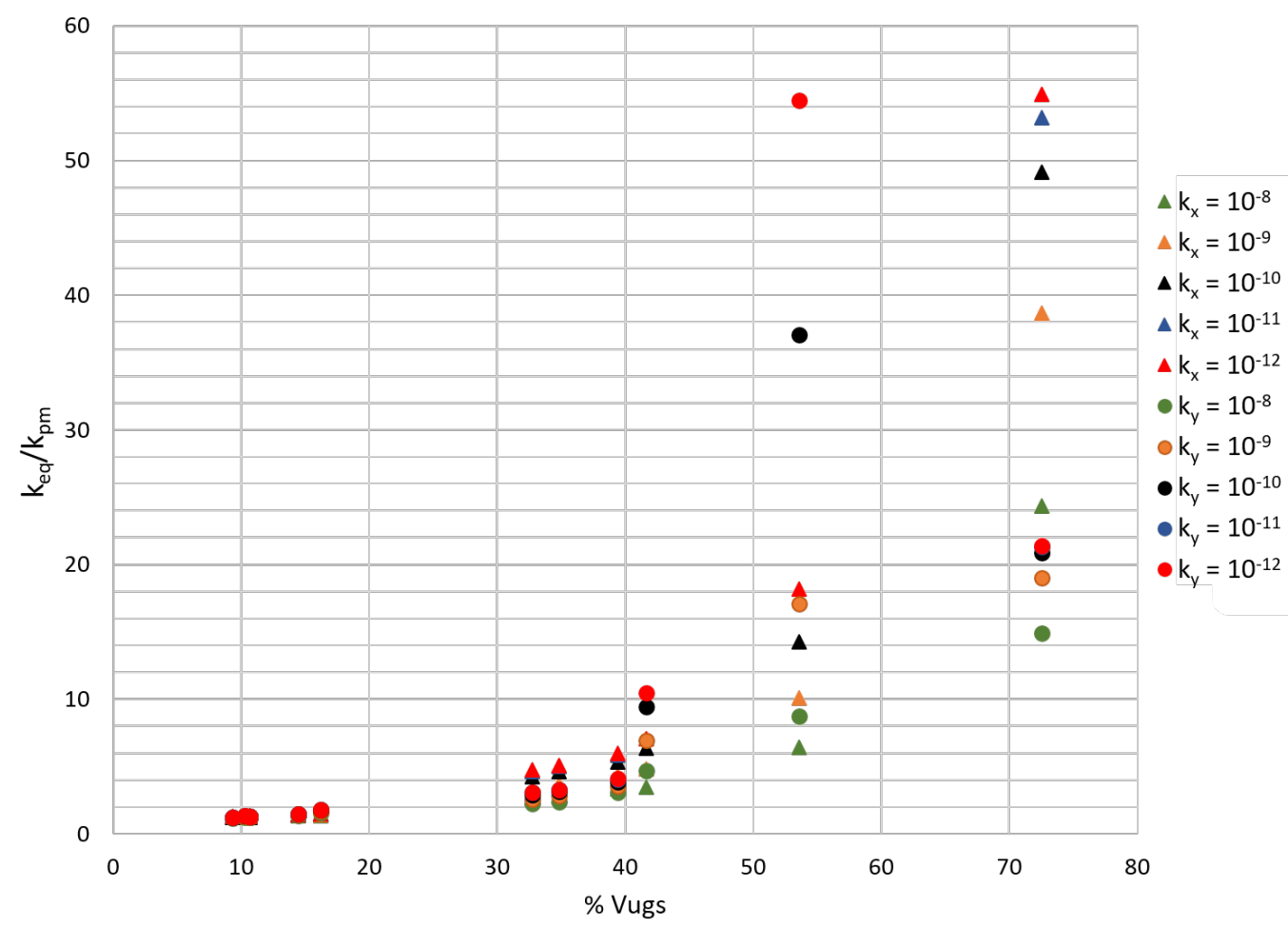

Figure 5.34: Equivalent permeability $\left(k_{e q}\right)$ for $\Delta P=6,894.76 \mathrm{~Pa}$, divided by each porous medium permeability input $\left(k_{p m}\right)$ as a function of voids of the cells shown in fig. 5.24 for sample $1 . k_{x}$ represents permeability of the porous medium when flow guidance is on the $\mathrm{x}$-direction and $k_{y}$ is when flow guidance is on the $\mathrm{y}$-direction. 
Turning to the second sample, fig. 5.35 shows the three selected and treated slices of the microtomography images. In the first line are the images for slice 1 which was taken from the top of Am05. In the left column, there are those that were processed to identify voids, while in the right are the images in which different filters were applied to increase the voids with the same methods applied in fig.5.24. It is possible to observe an essential difference between the two samples. Contrarily to the first sample, the vugs in the second are much smaller, and there is no formation of large cavities when the filters are applied.

The simulations were made with the same conditions imposed in the first case. The equivalent permeability values for the different sections of fig. 5.35 are much smaller than that of the first sample (Am08), possibly due to the scarcity of connections between the voids. Among the graphs of figs. 5.34 and 5.36, also note the difference in scale of equivalent permeability to that defined in the porous material.

These results are similar to those observed by Okabe and Blunt[29] in fig. 5.38 where they calculated the permeability using the Lattice Boltzmann method. When the correlation between macroporosity and computed permeability was analyzed, an increase in macro-porosity, in our case $\%$ vugs, also leads to an increases in the dispersion of computed permeability. This fact is attributed to the heterogeneous distributions of macropores. 


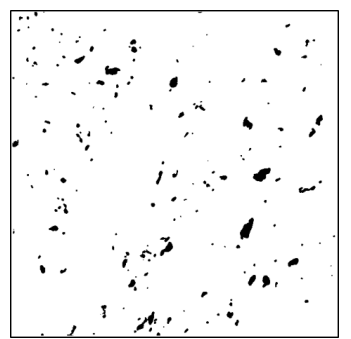

(a) $2.46 \%$ Voids.

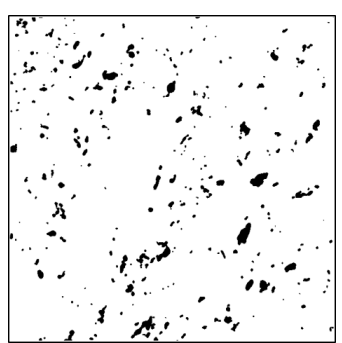

(b) $4.27 \%$ Voids.

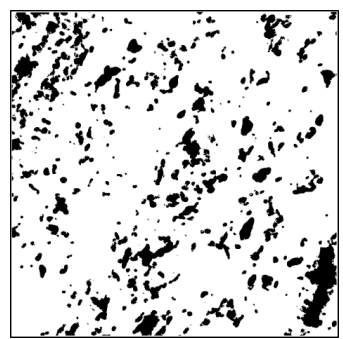

(c) $15.35 \%$ Voids.

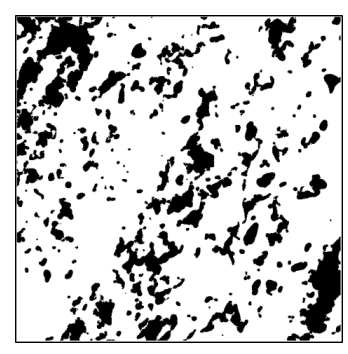

(d) $22.42 \%$ Voids.

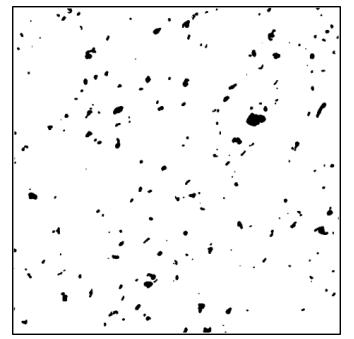

(e) $2.58 \%$ Voids

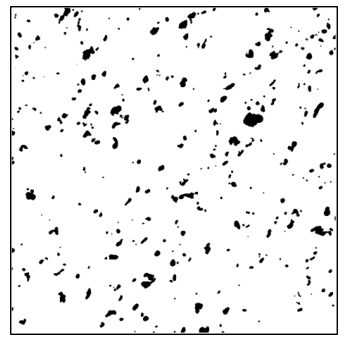

(f) $4.70 \%$ Voids.

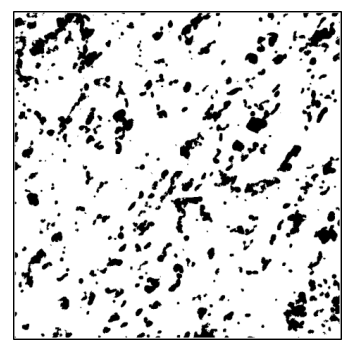

(g) $14.69 \%$ Voids.

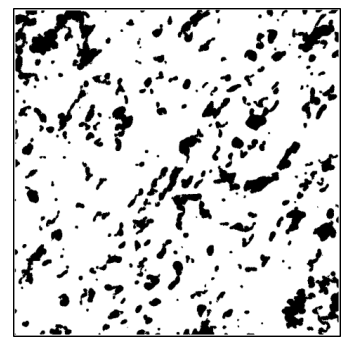

(h) $16.98 \%$ Voids.

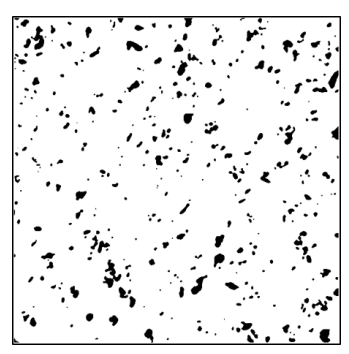

(i) $5.47 \%$ Voids.

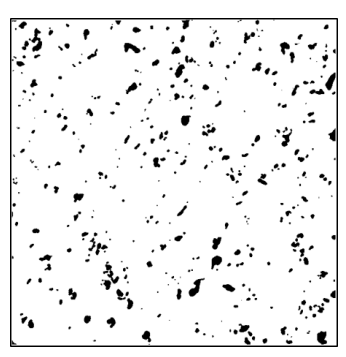

(j) $6.36 \%$ Voids.

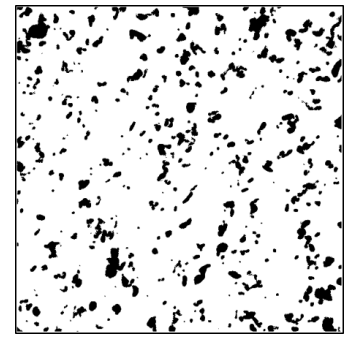

(k) $11.82 \%$ Voids.

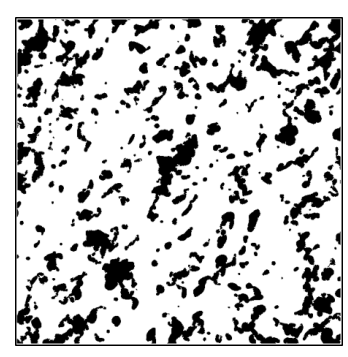

(l) $23.7 \%$ Voids.

Figure 5.35: Vugs enlargement on slice 1 (a-d), 2 (e-h) and 3 (i-l) of sample 2. In first row are the images for slice 1, which was taken from the upper part of the core sample 2. In the left column are those that have been processed to identify voids as done from 5.24 a to $5.24 \mathrm{~b}$, next to this was applied different thresholds to increase voids with same methods applied in fig. 5.24. 


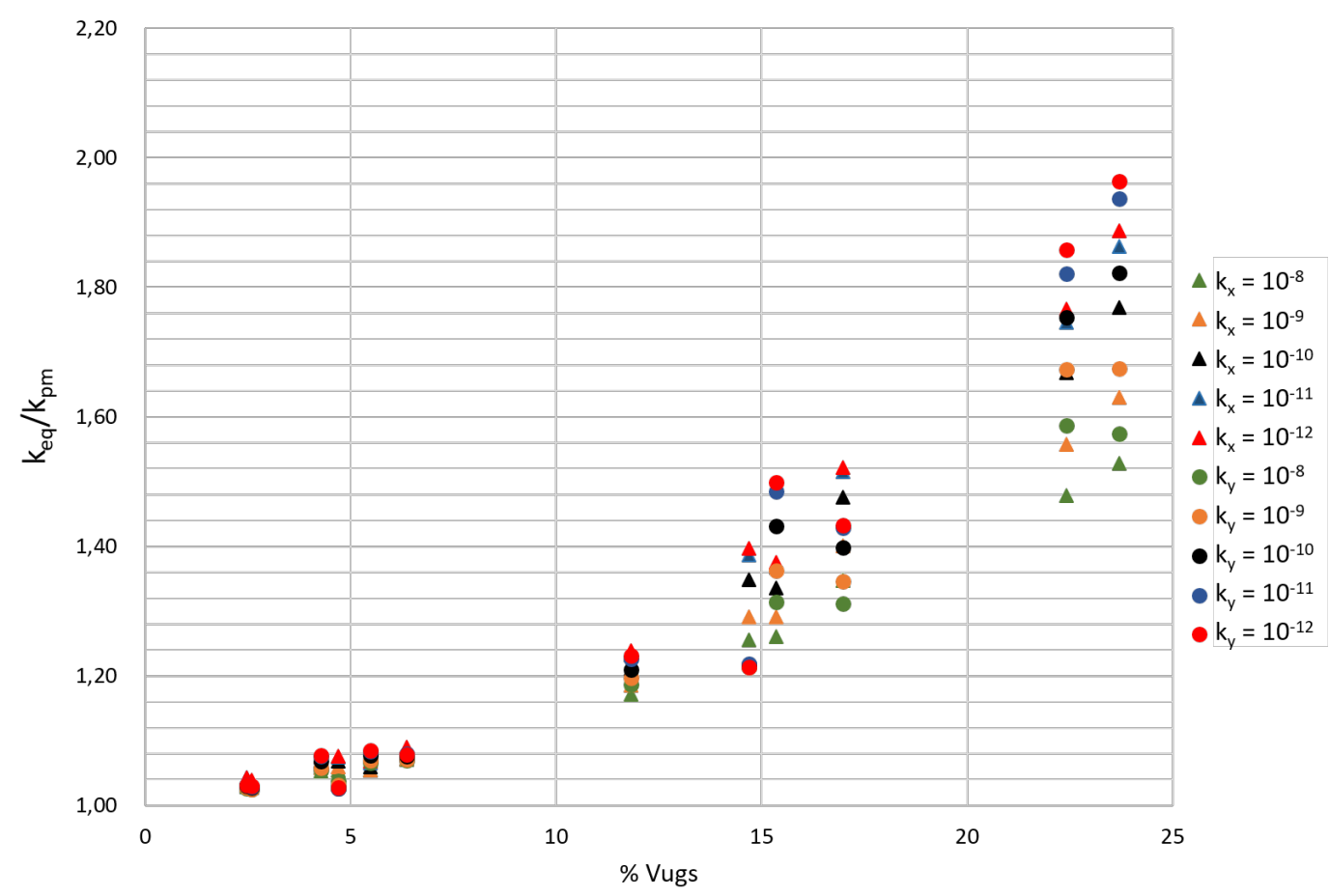

Figure 5.36: Equivalent permeability $\left(k_{e q}\right)$ for $\Delta P=6,894.76 \mathrm{~Pa}$, divided by each porous medium permeability $\left(k_{p m}\right)$ input as a function of the voids of the cells shown in fig. 5.35 for sample 2. $k_{x}$ represents permeability of porous media when flow guidance is on the $\mathrm{x}$-direction and $k_{y}$ is when flow guidance is on the y-direction.

(A)

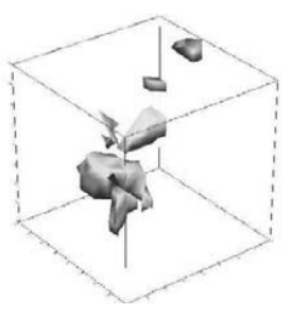

(B)

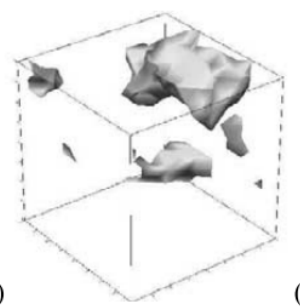

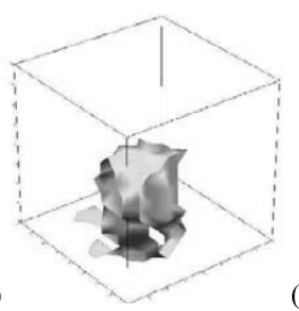

(C)
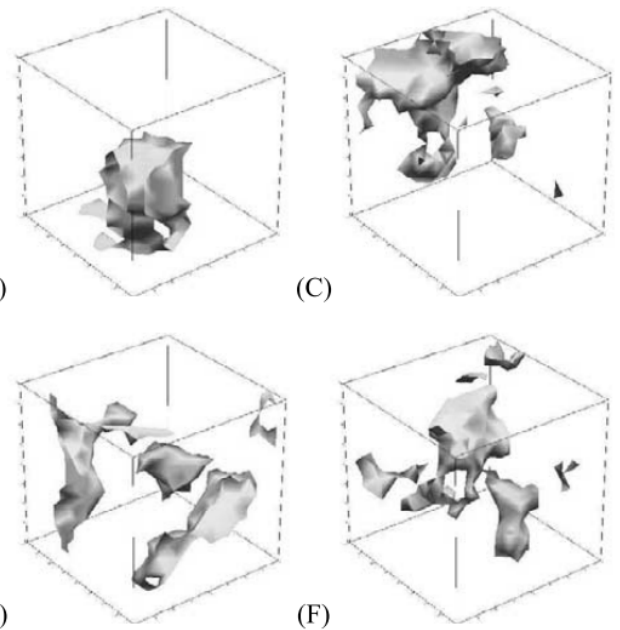

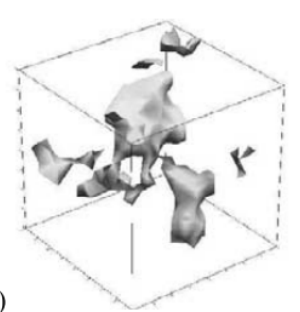

Figure 5.37: Subsets from the 3-D microtomography of macropores with different porosity values: (a) $\phi=0.05$, (b) $\phi=0.075$, and (c-f) $\phi=0.10$. From Okabe \& Blunt, 2007 


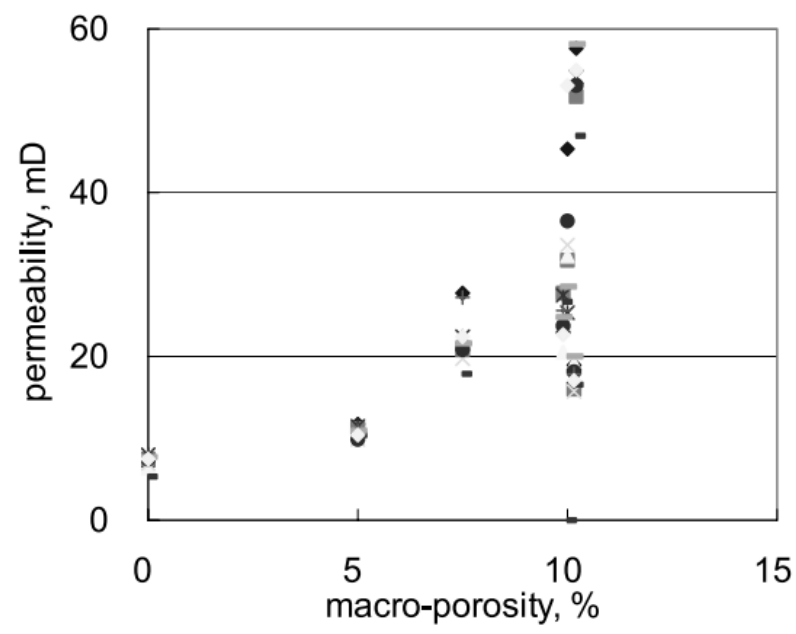

Figure 5.38: Variation of computed permeabilities in combined images of fig. 5.37 with different macroporosities. From Okabe \& Blunt, 2007.

In this section, the equivalent permeability data obtained with the simulation in coquinas samples is presented. The presence of vugs with different arrangements and sizes resulted in an anisotropic equivalent permeability. Sections with small isolated vugs, at most, doubled the permeability value. The presence of connected vugs significantly increases the equivalent permeability $k_{e q}$ to orders of magnitude higher than the matrix permeability $k_{p m}$.

This analysis did not show a direct or exponential relation between equivalent permeability and macroporosity. Aiming to identify some similarities between the simulations values, some relations presented in the literature are tested to estimate the permeability.

\subsection{3}

\section{Results analysis}

In order to determine a behavior for the equivalent permeability observed in fig. 5.32, the Carman-Kozeny's Equation[49] [50] was used. Equation 5-2 is traditionally used to relate the permeability $k$ to porosity $\phi$ and grain size $D_{p}$, providing a link between medium properties and flow resistance in pore channels[51].

$$
k=\alpha D_{p}^{2} \frac{\phi^{3}}{(1-\phi)^{2}}
$$

where $\alpha$ includes proportionality parameters and unit factor.

It was used an approximation of eq. 5-2 substituting the parameters $\alpha$ and $D_{p}$ for coefficient "a", as a data fit curve for slice 1 of sample 1 . However, it can be seen in fig. 5.39 that the fit does not have coefficients " $a$ " with close 
values for $x$ and $y$-directions, since the properties of the analyzed section do not change within the same portion, the equation does not represent the difference in values due to change of direction of flow and neither transmits the influence of the vuggy geometry to the result.

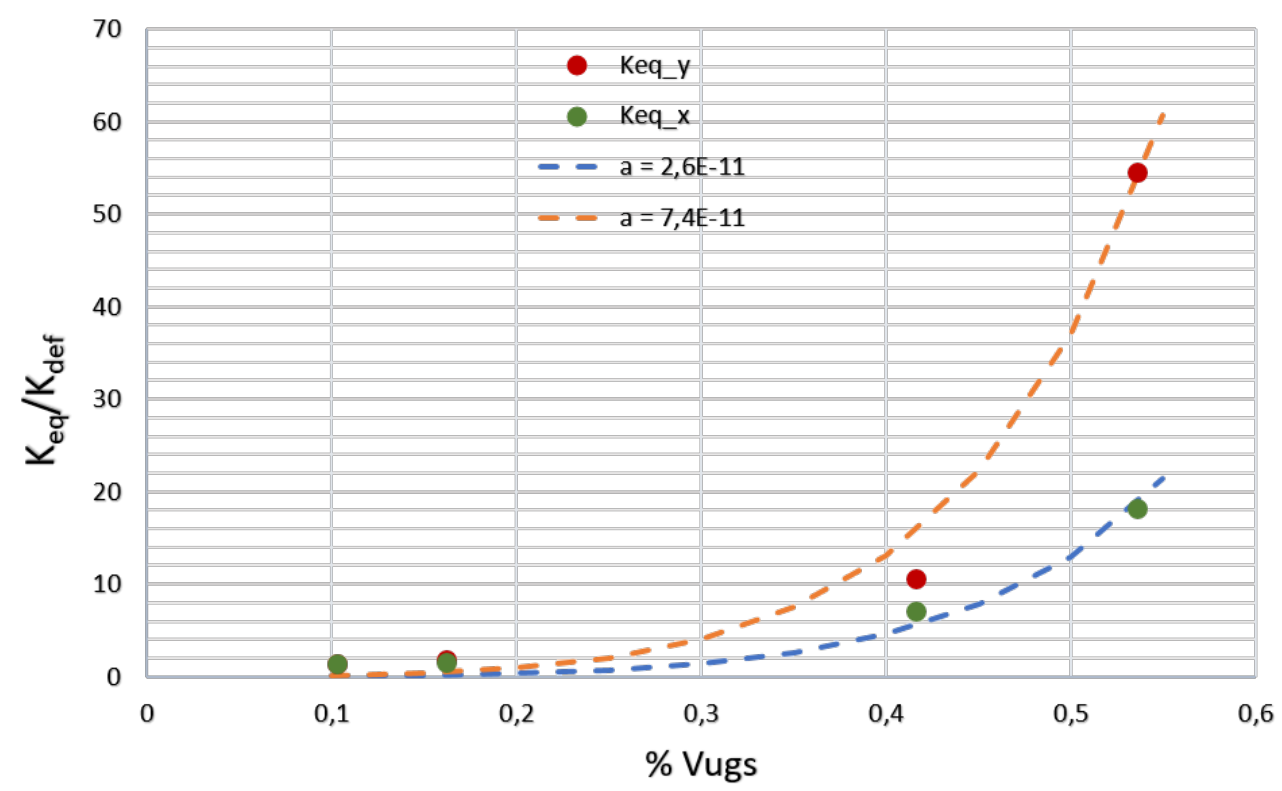

Figure 5.39: Curve adjustment based on the Carman-Kozeny equation with $\Delta P=6,894.76 \mathrm{~Pa}$ and $k_{p m}=10^{-12} \mathrm{~m}^{2}$.

In the final analysis, a method to determine the equivalent permeability in porous media with large vugs that reach similar results with the observed in figs. 5.34 and 5.36 is required. Thus, a theoretical calculation that describes the phenomenon observed in this numerical study can be used as a primary method to predict the permeability in porous media with macropores.

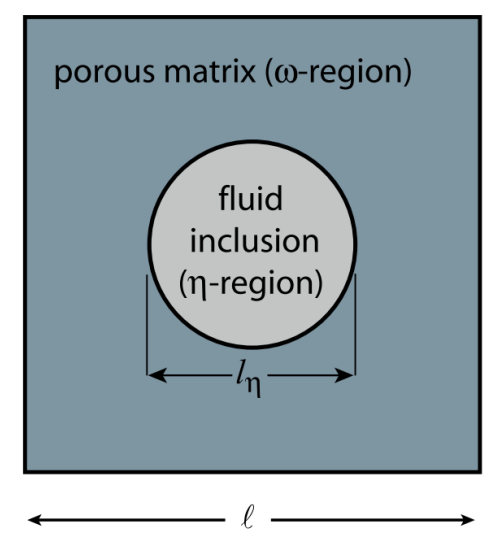

Figure 5.40: Representative cell of a 2D vuggy porous media. From Golfier et al., 2015. 
Golfier et al. [27] proposed a relation between equivalent permeability and circular inclusion as eq. 5-3 for a two-dimensional vuggy system. Figure 5.40 illustrates the representative cell which eq. 5-3 was formulated. A comparison between our computational values and the results for this equation was performed and presented in tab. 5.2. For this calculation, an equivalent vuggy media was considered to fill out eq. 5-3 that is based in a circular inclusion model, $\eta$-region, surrounded by a square-shaped porous matrix, $\omega$-region.

$$
\begin{gathered}
K_{e q, v u g}^{*}=\frac{K_{\omega}\left[R^{2}\left(1+\phi_{\eta}\right)-2\left(\phi_{\eta}-1\right) K_{\omega}-3 R\left(1+\phi_{\eta}\right) \alpha \sqrt{K_{\omega}}\right]}{R^{2}\left(1-\phi_{\eta}\right)+2\left(\phi_{\eta}+1\right) K_{\omega}+3 R\left(\phi_{\eta}-1\right) \alpha \sqrt{K_{\omega}}} . \\
R=\sqrt{\frac{\phi_{\eta}}{\pi}} l .
\end{gathered}
$$

where $R$ represents the vug radius, $K_{\omega}$ the porous matrix permeability, $\phi_{\eta}$ the area fraction of the vug and $\alpha=\sqrt{1 / \varepsilon_{\beta}}$ with $\varepsilon_{\beta}=0.3$ denoting the porosity inside porous matrix.

Figure 5.41 illustrates a representative unit cell created based on an area fraction of a slice of the core samples under investigation. Using the fraction of vug area, an equivalent circular vug was built. Then, with this information extracted from this perfect geometry, it is possible to calculate the equivalent permeability through equations 5-3 and 5-4. In this case, one large vug is created to substitute all tiny macropores in a microtomography section.

Table 5.2 presents the comparison between both methods, where it is possible to observe a similarity in the results.

\begin{tabular}{|c|c|c|c|}
\hline Direction & Computed $k_{e q} / k_{p m}$ & Calculated $k_{e q} / k_{p m}$ & Difference $(\%)$ \\
\hline $\mathrm{X}$ & 1.28 & 1.23 & 3.6 \\
\hline $\mathrm{Y}$ & 1.33 & 1.23 & 7.6 \\
\hline
\end{tabular}

Table 5.2: Comparison between calculated equivalent permeability $\left(k_{e q}\right)$ using eq.5-3 and equivalent permeability $\left(k_{e q}\right)$ for a $\mu C T$ image divided by porous region permeability $\left(k_{p m}\right)$.

A better representation could be made decentralizing this measure, partitioning each section in many sub-samples and calculating theoretical equivalent permeability with the intention of bringing the representative model closer to the base image. In this case, the reservoir section contains distinct blocks of varying permeabilities. 


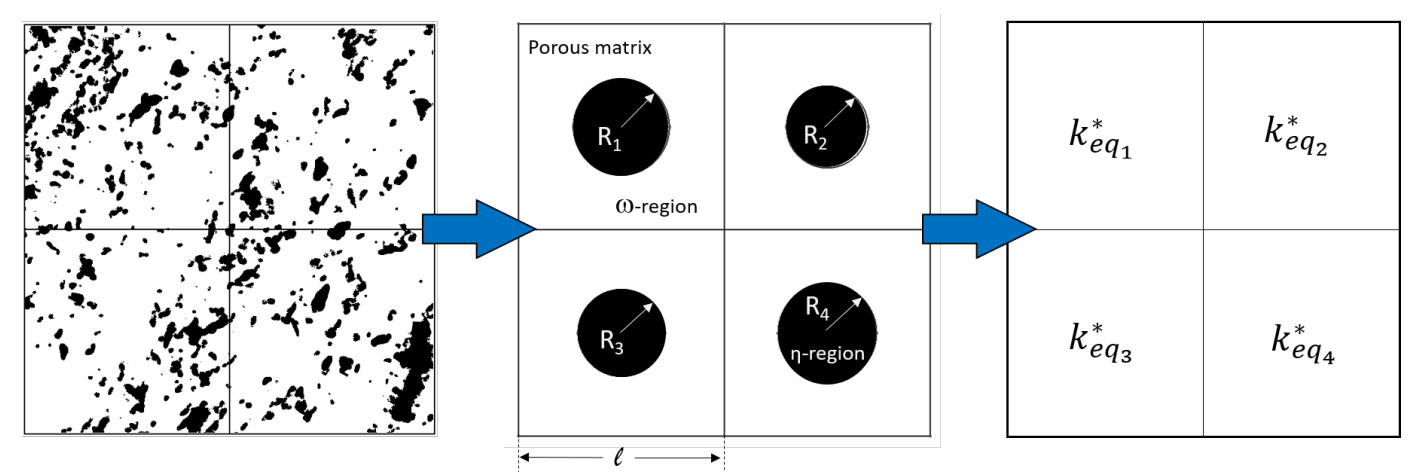

Figure 5.41: Procedure adopted to create an equivalent vug system.

Permeability-averaging techniques are commonly used to determine an appropriate average permeability to represent an equivalent homogeneous system. With this in hand, an equivalent global permeability was then determined by combining weighted and harmonic-average. An expression for weighted and harmonic-average permeability of blocks with the same thickness for four subdivisions, presented in fig. 5.41, is:

$$
k_{\text {avg }}=\frac{\left(k_{1}+k_{3}\right)\left(k_{2}+k_{4}\right)}{\sum_{i=1}^{4} k_{i}}
$$

Although the geometric-average permeability is often used in carbonates, this method did not represent very well the simulation results because it retains both high and low values and what has been observed is that high permeability sections have a great impact on the output flow rate, mainly when the voids are interconnected.

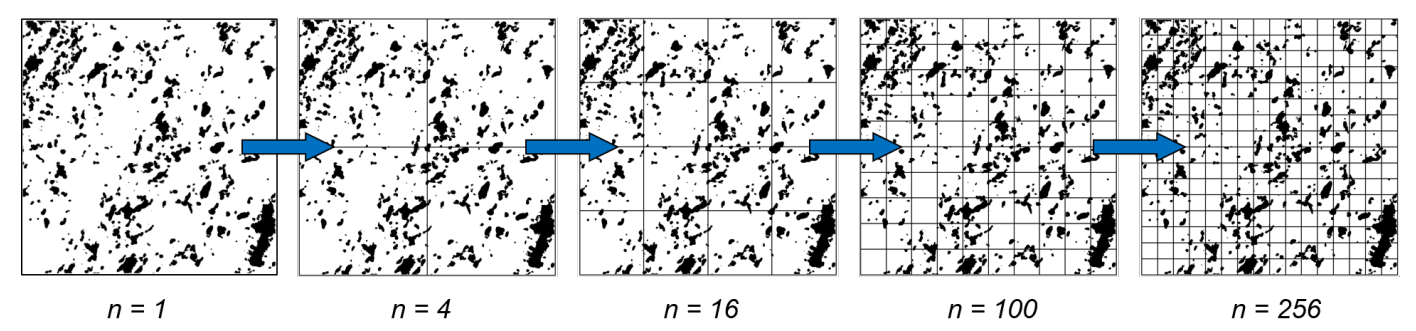

Figure 5.42: Size partitioning and number of sub-samples for a slice of sample Am05.

Figure 5.42 shows how the sample was partitioned in sub-samples created for this analysis. Figure 5.43 illustrates the comparison between computational results for the domains shown in fig. 5.45 , flow in $x$-direction and the averagepermeability solution where the variation of $k_{e q} / k_{p m}$ is represented as a 
function of the macroporosity for the porous matrix permeability of $k_{p m}=$ $10^{-12} \mathrm{~m}^{2}$.

Analyzing the results, it is possible to observe a similarity between numerical and theoretical model results, except for large values of macroporosity for which the relation requires more partitions. For $\phi_{\eta}<20 \%$, good agreement between both results is achieved for small values of $n$ sub-samples. For large values of \% vugs, equivalent medium approximation needs more $\mathrm{n}$ divisions. In this case, 100 partitions worked very well for point 7 and 256 sub-samples have the lowest deviation for points 8 and 9 . However, for point 9 the approximation fails. In this case, for $n=100$ the solution is far from the computational and for $\mathrm{n}=256$ the value passes the target.

By the results obtained, it was possible estimate the equivalent permeability based on flow simulations in virtual representations of Coquinas samples. The methodology developed to estimate equivalent permeability from segmented images is a combination of an equation available in literature and partition of these images into sub-samples. 


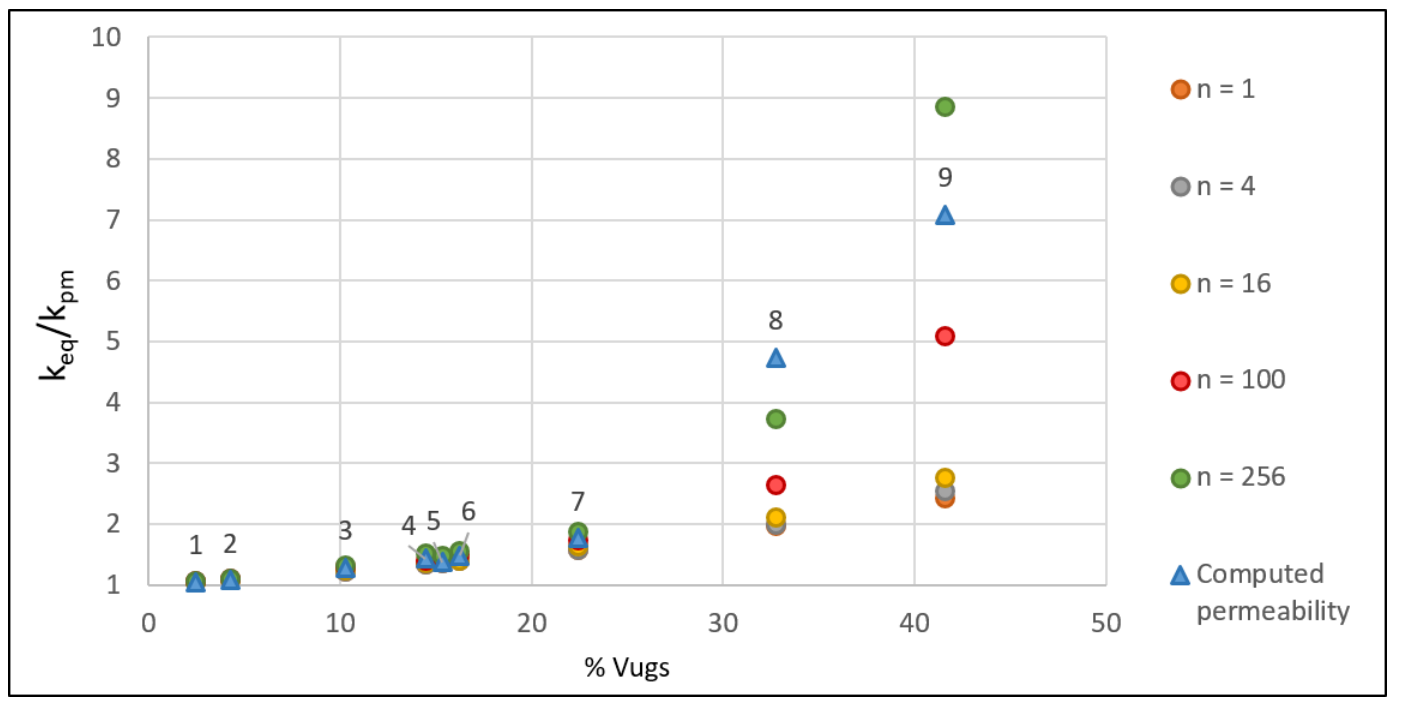

Figure 5.43: Equivalent homogeneous permeabilities for $\Delta P=6,894.76 \mathrm{~Pa}$, different slices of core samples and $\mathbf{n}$ sub-samples.

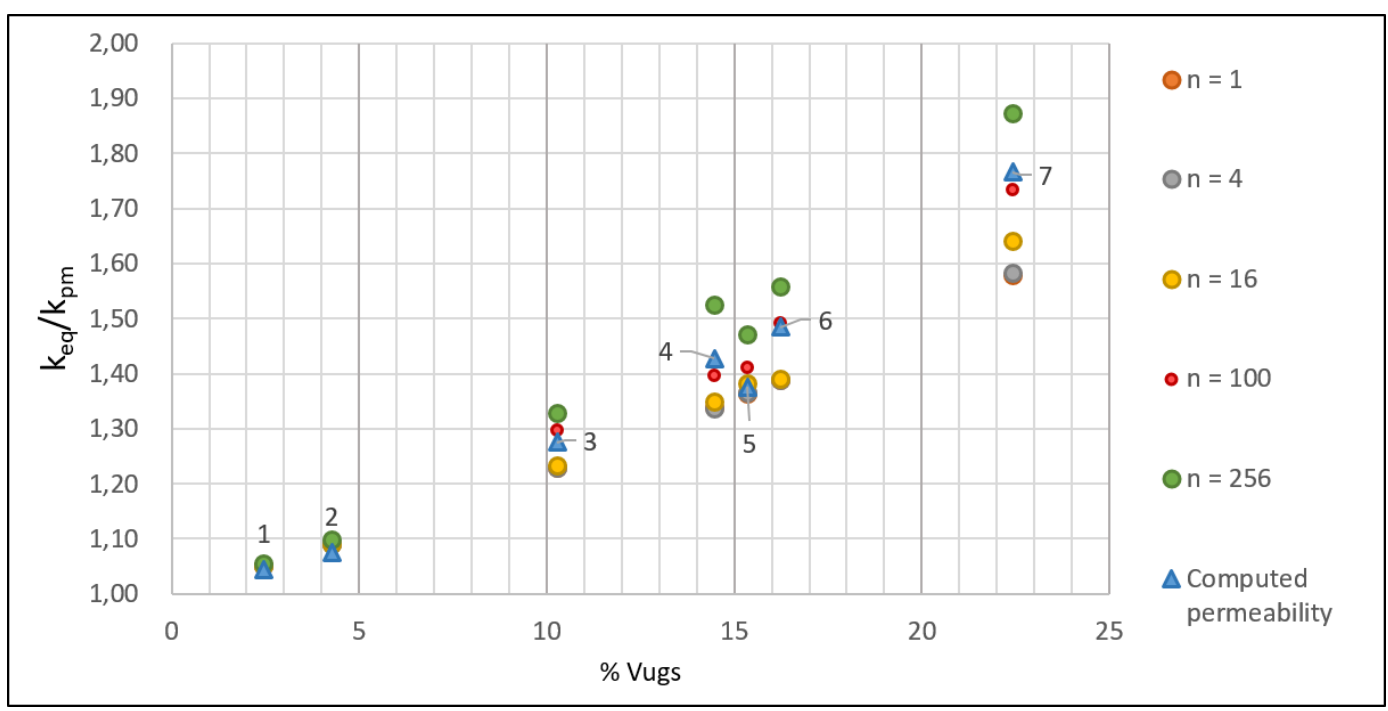

Figure 5.44: Equivalent homogeneous permeability for macroporosity under $25 \%$. 


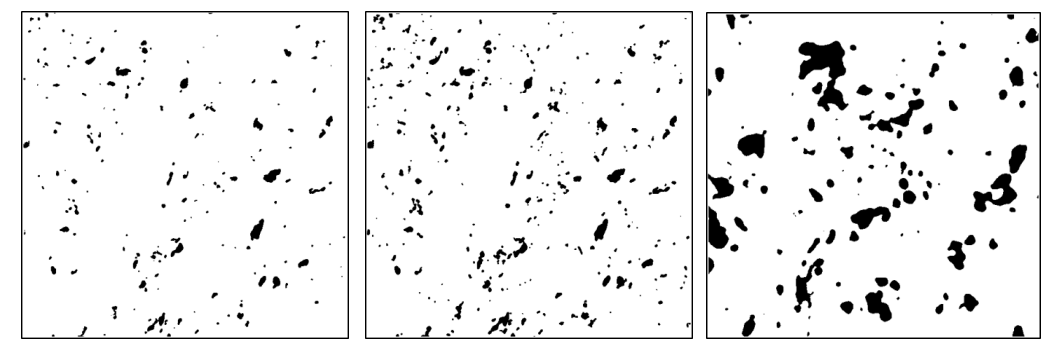

(a) 1

(b) 2

(c) 3

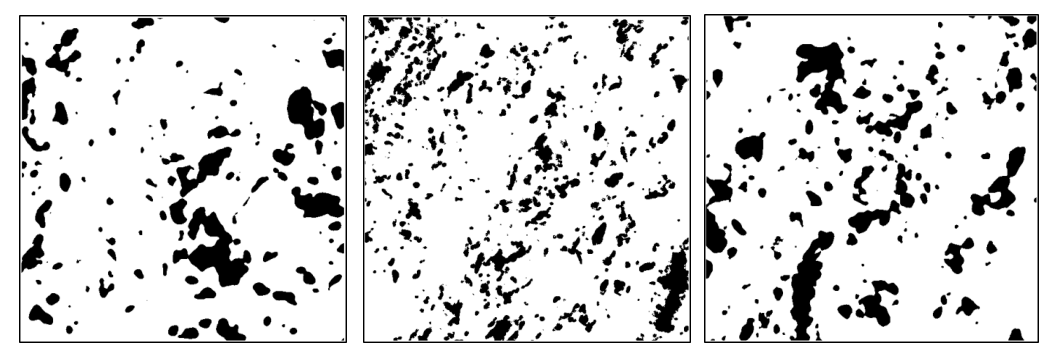

(d) 4

(e) 5

(f) 6

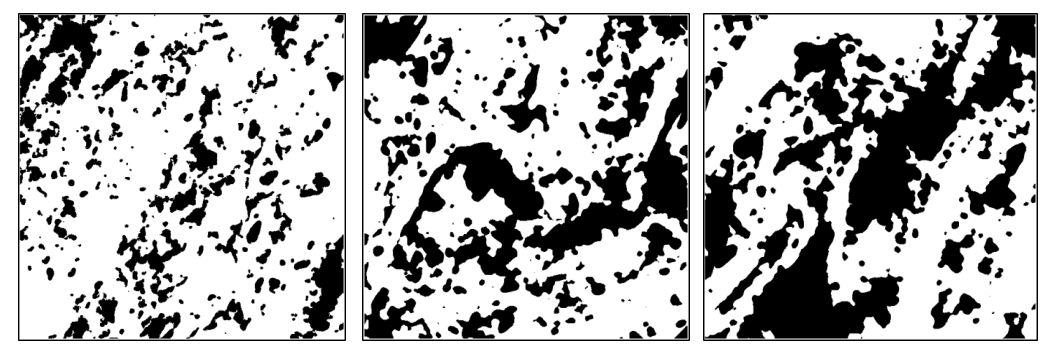
(g) 7
(h) 8
(i) 9

Figure 5.45: Images used to build graphic 5.43. Figures 5.45a, 5.45b, 5.45e and $5.45 \mathrm{~g}$ were extracted from sample $2, \mathrm{Am} 05$. The others are images based on sample 1, Am08. 


\section{6 \\ Discussion and conclusions}

In this work, the effect of the macropores presence on the flow in porous media was studied by solving the Brinkman's equation. To achieve the objective of develop a methodology to estimate the permeability in heterogeneous porous medium, a numerical model was implemented using the finite element method and the library of FEniCS platform for solving partial differential equations.

This study parametrically investigated the effect of vug area and structure on the flow in carbonate rock samples. Before that, it was necessary to carry out the validation of the model and to analyze the interface condition between free flow region and permeable material.

Based on the finite element method, we derived a model using DarcyBrinkman equation to simulate flow through heterogeneous porous media. The study of coupled parallel flows within a two-region domain elucidated the mode in which Brinkman equation is able to replicate the flow between the transition zone by comparison with the classic Beavers and Joseph[5] analysis.

Despite the lack of equivalence concerning the effect of the porous bounding on the mass flow rate increment, the presence of channels that connect opposite faces in our model showed consistent results relative to the velocity profile and the velocity slip value.

After these analysis, we were able to focus on the main purpose of this work, which was the estimation of the permeability in representative sections of carbonate rock samples. For this, a 2D geometry representative model was built from a tomography image reading.

In order to upscale the equivalent permeability tensor, flow in two different directions through the discrete segmented regions of the sample was imposed. The presence of vugs with different sizes and locations resulted in an anisotropic scaled-up permeability. The velocity magnitude field and the back-calculated permeabilities clearly showed a preferential path aligned with the direction of vugs and connectivity. This relation was evidenced when the vugs were enlarged, by eroding the holes with the threshold technique. This method divided the pixels of the samples images into two distinct regions by choosing a cut off value.

The numerical solution of the flow in slices and manipulated images of 
carbonate samples did not show a simple relation between macroporosity and permeability, as expected for this type of rock. Next, an analytic formula that describes the phenomenon observed was searched and an equation to determine the permeability for vugs of circular shape was tested. For the purpose of using this equation, an equivalent circular shape was calculated based on the percentual of the free area of the image. The comparison between computed permeability and analytical for one large circular vug showed promising values.

For achieving better approximations, some images studied were divided into blocks. For each block, an equivalent circular inclusion was calculated in order to analytically estimate the permeability. These values for sub-slices were upscaled using the weighted and the harmonic average. The mean value of the estimated permeability for images, using this technique, reached extremely close values to the previously numerically determined, except at exceedingly large values of vug area fraction. These large vug area values are extreme cases that no longer match the real case. The equivalent permeabilities obtained from the two approaches presented less than $3 \%$ of deviation for macroporosity under $25 \%$ and sub-divisions with 100 blocks.

\section{1 \\ Future works}

There are some open questions regarding the study of carbonate karst reservoirs due to their high heterogeneity in small sections. The equivalent permeability as a function of the geometry and structure of the vugs could be interesting as an input parameter in simulators. For this to be feasible, it would be necessary to analytically determine the equivalent permeability for a wide range of macroporosities, using various combinations of fracture and vug models. Then, a major step would be to measure and understand how the flow is affected by the direction and angles of the void spaces.

In relation to the coupled parallel flow and its surface condition, the work of Beavers and Joseph is a representative physical experiment on the coupling of the single-phase flow problem. It was difficult to reproduce numerically the same experiments, mainly due to the relevance of the structure characteristic of porous media. Thus, further analysis of the relative increase of flow through the channel due to sliding with the porous surface is required.

Another important point is to expand the analysis to the 3D model in order to provide a more accurate permeability estimate. There are many benefits involving a 3D approach, since it would incorporate more precisely the effects of the connection between pores, allowing for a more exact comparison of numerical results with experimental data. 
When upscaling permeability from a fine scale model to a coarse scale, several considerations should be taken into account. One step forward in this direction could be the use of permeability and microporosity measured in laboratory from small pieces of core samples in order to allow the comparison of the modelled results with reservoirs based on the geological statistical database of wells. 


\section{Bibliography}

[1] Schlumberger carbonates. http://www.slb.com/services/.. /carbonates. aspx. Accessed: 2017-11-12.

[2] Rodolfo Oliveira and Marcio da Silveira Carvalho. Finite element method applied to flow in heterogeneous porous media. Departamento de Engenharia Mecânica. Pontifícia Universidade Católica do Rio de Janeiro, 2014.

[3] Jun Yao and Zhao-Qin Huang. Fractured Vuggy Carbonate Reservoir Simulation. Springer, 2017.

[4] T Arbogast, DS Brunson, SL Bryant, and JW Jennings Jr. A preliminary computational investigation of a macro-model for vuggy porous media. In Developments in Water Science, volume 55, pages 267-278. Elsevier, 2004.

[5] Gordon S Beavers and Daniel D Joseph. Boundary conditions at a naturally permeable wall. Journal of fluid mechanics, 30(1):197-207, 1967.

[6] Philip Geoffrey Saffman. On the boundary condition at the surface of a porous medium. Studies in applied mathematics, 50(2):93-101, 1971.

[7] Andro Mikelić and Willi Jäger. On the interface boundary condition of beavers, joseph, and saffman. SIAM Journal on Applied Mathematics, 60(4):1111-1127, 2000.

[8] ZF Tian, C Xu, and PA Dowd. Numerical simulation of flows in a channel with impermeable and permeable walls using finite volume methods. Flow and Transport in Subsurface Environment, pages 119-140, 2018.

[9] IP Jones. Low reynolds number flow past a porous spherical shell. In Mathematical proceedings of the Cambridge philosophical society, volume 73, pages 231-238. Cambridge University Press, 1973.

[10] Qianlong Liu and Andrea Prosperetti. Pressure-driven flow in a channel with porous walls. Journal of fluid mechanics, 679:77-100, 2011. 
[11] Gordon S Beavers, Ephraim M Sparrow, and Ronald Allen Magnuson. Experiments on coupled parallel flows in a channel and a bounding porous medium. Journal of Basic Engineering, 92(4):843-848, 1970.

[12] S Richardson. A model for the boundary condition of a porous material. part 2. Journal of Fluid Mechanics, 49(2):327-336, 1971.

[13] GS Beavers, EM Sparrow, and BA Masha. Boundary condition at a porous surface which bounds a fluid flow. AIChE Journal, 20(3):596-597, 1974.

[14] B Goyeau, D Lhuillier, D Gobin, and MG Velarde. Momentum transport at a fluid-porous interface. International Journal of Heat and Mass Transfer, 46(21):4071-4081, 2003.

[15] DA Nield. The beavers-joseph boundary condition and related matters: a historical and critical note. Transport in porous media, 78(3):537, 2009.

[16] RE Larson and JJL Higdon. Microscopic flow near the surface of twodimensional porous media. part 1. axial flow. Journal of Fluid Mechanics, 166:449-472, 1986.

[17] HC Brinkman. A calculation of the viscosity and the sedimentation constant for solutions of large chain molecules taking into account the hampered flow of the solvent through these molecules. Physica, 13:447448, 1947.

[18] Jie He, John E Killough, F Fadlelmula, M Mohamed, Michael Fraim, et al. A unified finite difference model for the simulation of transient flow in naturally fractured carbonate karst reservoirs. In SPE Reservoir Simulation Symposium. Society of Petroleum Engineers, 2015.

[19] KR Rajagopal. On a hierarchy of approximate models for flows of incompressible fluids through porous solids. Mathematical Models and Methods in Applied Sciences, 17(02):215-252, 2007.

[20] GI t Taylor. A model for the boundary condition of a porous material. part 1. Journal of Fluid Mechanics, 49(2):319-326, 1971.

[21] Graham Neale and Walter Nader. Practical significance of brinkman's extension of darcy's law: coupled parallel flows within a channel and a bounding porous medium. The Canadian Journal of Chemical Engineering, 52(4):475-478, 1974.

[22] HC Brinkman. On the permeability of media consisting of closely packed porous particles. Flow, Turbulence and Combustion, 1(1):81, 1949. 
[23] Michael Le Bars and M Grae Worster. Interfacial conditions between a pure fluid and a porous medium: implications for binary alloy solidification. Journal of Fluid Mechanics, 550:149-173, 2006.

[24] Peter Popov, Yalchin Efendiev, and Guan Qin. Multiscale modeling and simulations of flows in naturally fractured karst reservoirs. Communications in computational physics, 6(1):162, 2009.

[25] Astrid Fossum Gulbransen, Vera Louise Hauge, Knut-Andreas Lie, et al. A multiscale mixed finite element method for vuggy and naturally fractured reservoirs. Spe Journal, 15(02):395-403, 2010.

[26] Guan Qin, Linfeng Bi, Peter Popov, Yalchin Efendiev, Magne Espedal, et al. An efficient upscaling procedure based on stokes-brinkman model and discrete fracture network method for naturally fractured carbonate karst reservoirs. In International Oil and Gas Conference and Exhibition in China. Society of Petroleum Engineers, 2010.

[27] Fabrice Golfier, D Lasseux, and M Quintard. Investigation of the effective permeability of vuggy or fractured porous media from a darcy-brinkman approach. Computational Geosciences, 19(1):63-78, 2015.

[28] Marcin Krotkiewski, Ingeborg S Ligaarden, Knut-Andreas Lie, and Daniel W Schmid. On the importance of the stokes-brinkman equations for computing effective permeability in karst reservoirs. Communications in Computational Physics, 10(5):1315-1332, 2011.

[29] Hiroshi Okabe and Martin J Blunt. Pore space reconstruction of vuggy carbonates using microtomography and multiple-point statistics. Water Resources Research, 43(12), 2007.

[30] Schlumberger the oilfield glossary: Where the oil field meets the dictionary. https://www.glossary.oilfield.slb.com/en/Terms/p/ porous_medium.aspx. Accessed: 2018-10-18.

[31] Richard C Selley and Stephen A Sonnenberg. Geologia do Petróleo: Tradução da 3a edição. Elsevier Brasil, 2016.

[32] Adalberto José Rosa, Renato de Souza Carvalho, and José Augusto Daniel Xavier. Engenharia de reservatórios de petróleo. Interciência, 2006.

[33] How to upscale permeability. http://www.epgeology.com/ static-modeling-f39/how-upscale-permeability-t6045.html.

Accessed: 2019-02-20. 
[34] Gary Nichols. Sedimentology and stratigraphy. John Wiley \& Sons, 2009.

[35] GJS Terra, AR Spadini, AB França, CL Sombra, EE Zambonato, LC da S Juschaks, LM Arienti, MM Erthal, M Blauth, MP Franco, et al. Classificação de rochas carbonáticas aplicável às bacias sedimentares brasileiras. Boletin Geociencias Petrobras, 18:9-29, 2010.

[36] Erik Flügel. Microfacies of carbonate rocks: analysis, interpretation and application. Springer Science \& Business Media, 2013.

[37] Denise Prado Kronbauer. Determinação de propriedades petrofísicas de rochas reservatórios usando modelos de redes de poros. $\mathrm{PhD}$ thesis, Universidade Federal de Santa Catarina, 2014.

[38] M.C.O. Lima, L.P. Martins, A. Orlandi, M. Mendes, L.F. Borghi, and P. Couto. Análise do espaço poroso de coquinas da formação morro do chaves através de ressonância magnética nuclear e microtomografia de raio-x. In CONGRESSO BRASILEIRO DE GEOLOGIA, volume 49, 2018.

[39] Philip W Choquette and Lloyd C Pray. Geologic nomenclature and classification of porosity in sedimentary carbonates. AAPG bulletin, 54(2):207-250, 1970.

[40] Clyde H Moore. Carbonate diagenesis and porosity, volume 46. Elsevier, 1989.

[41] O. C. Zienkiewicz, J. Z. Zhu, and R. L. Taylor. The Finite Element Method: Its Basis and Fundamentals., volume Seventh edition. Butterworth-Heinemann, 2013.

[42] Thomas JR Hughes. The finite element method: linear static and dynamic finite element analysis. Courier Corporation, 2012.

[43] Hans Petter Langtangen and Anders Logg. Solving pdes in python the fenics tutorial volume i, 2016.

[44] Robert W. Fox, Philip J. Pritchard, and Alan T. McDonald. Introdução à mecânica dos fluidos. Rio de Janeiro : LTC, c2014., 2014.

[45] Douglas N Arnold and Anders Logg. Periodic table of the finite elements. SIAM News, 47(9):212, 2014. 
[46] GG Garcia, MH Henriques, SAST Figueiredo, KAL Santos, MVS Dantas, AJV Garcia, and E Roemers-Oliveira. Análise tafonômica das associações registradas na formação morro do chaves (bacia sergipe-alagoas) na definição da qualidade de reservatório. In CONGRESSO BRASILEIRO DE GEOLOGIA, volume 48, 2016.

[47] Caroline A Schneider, Wayne S Rasband, and Kevin W Eliceiri. Nih image to imagej: 25 years of image analysis. Nature methods, 9(7):671, 2012.

[48] Cyprien Soulaine. On the origin of darcy's law, 2015.

[49] Josef Kozeny. Uber kapillare leitung der wasser in boden. Royal Academy of Science, Vienna, Proc. Class I, 136:271-306, 1927.

[50] Philip Crosbie Carman. Fluid flow through granular beds. Trans. Inst. Chem. Eng., 15:150-166, 1937.

[51] Antonio Costa. Permeability-porosity relationship: A reexamination of the kozeny-carman equation based on a fractal pore-space geometry assumption. Geophysical Research Letters, 33(2), 2006. 


\section{A}

\section{FEniCS Algorithms}

Scripts A.1: Main Program

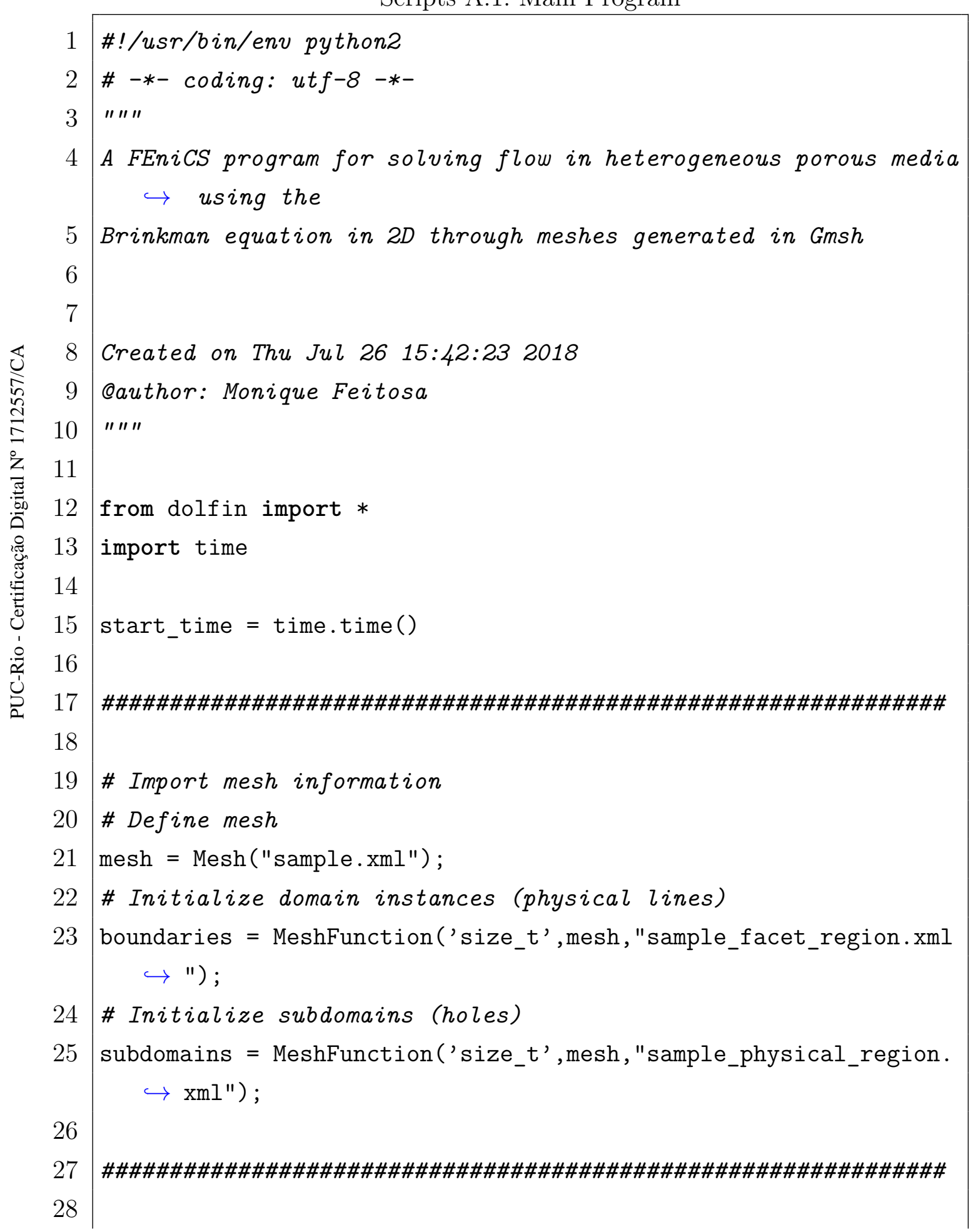


29

30

31

32

33

34

35

36

37

38

39

40

41

42

43

44

45

46

47

\# Function space over the mesh

$\mathrm{V}$ = VectorElement (' $\mathrm{CG}$ ' , mesh.ufl_cell ()$, 2)$

$Q=$ FiniteElement $\left({ }^{\prime} \mathrm{CG} \mathrm{G}^{\prime}\right.$, mesh.ufl_cell ()$\left., 1\right)$

Element $=\mathrm{V} * \mathrm{Q}$

$\mathrm{W}=$ FunctionSpace (mesh,Element)

\# Define variational problem

$(u, p)=$ TrialFunctions $(W)$

$(v, q)=$ TestFunctions $(W)$

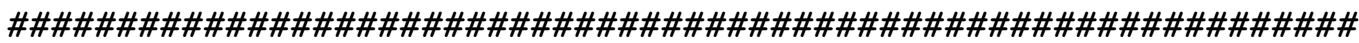

\# Material properties

$\mathrm{mu}=0.001002$ \# Water viscosity [Pa.s]

$\mathrm{k}=1 \mathrm{E}-14 \quad$ \# Porous media permeability [m2] $(1$ Darcy $=E-12 \mathrm{~m} 2)$

$\mathrm{pr}=6894.76$ \# Pressure $=1$ psi

pin $=2 * \mathrm{pr}$ \# Imposed pressure at the entrance [Pa]

pout $=\mathrm{pr} \#$ Imposed pressure on output [Pa]

\# Define expressions used in variational forms

$\mathrm{dp}=$ pin-pout

$\mathrm{u}_{\text {_in }}=$ Constant $((0.0))$ \#Initial velocity in $x[\mathrm{~m} / \mathrm{s}]$

$\mathrm{f}=$ Constant $((0.0,0.0))$ \#External force

$\mathrm{n}=$ FacetNormal (mesh) \#Normal vector to mesh

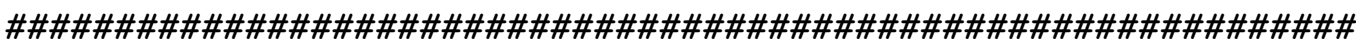

\# Define boundary conditions

$\mathrm{bc1}=\operatorname{DirichletBC}(\mathrm{W} \cdot \operatorname{sub}(0) \cdot \operatorname{sub}(0)$, Constant $(0.0)$, boundaries, 7$)$

$\mathrm{bc} 2=\operatorname{DirichletBC}(\mathrm{W} \cdot \operatorname{sub}(0) \cdot \operatorname{sub}(0)$, Constant $(0.0)$, boundaries , 8)

$\mathrm{bc3}=\operatorname{DirichletBC}(\mathrm{W} \cdot \operatorname{sub}(0) \cdot \operatorname{sub}(0)$, Constant $(0.0)$, boundaries, 9$)$

$\mathrm{bc} 4=\operatorname{DirichletBC}(W \cdot \operatorname{sub}(0) \cdot \operatorname{sub}(0)$, Constant $(0.0)$, boundaries, 10$)$

$b c s=[b c 1, b c 2, b c 3, b c 4]$

\# Define measures associated with the boundaries and holes

$\mathrm{ds}=$ Measure('ds', domain=mesh, subdomain_data = boundaries)

$\mathrm{dx}=$ Measure ('dx', domain=mesh, subdomain_data $=$ subdomains $)$ 


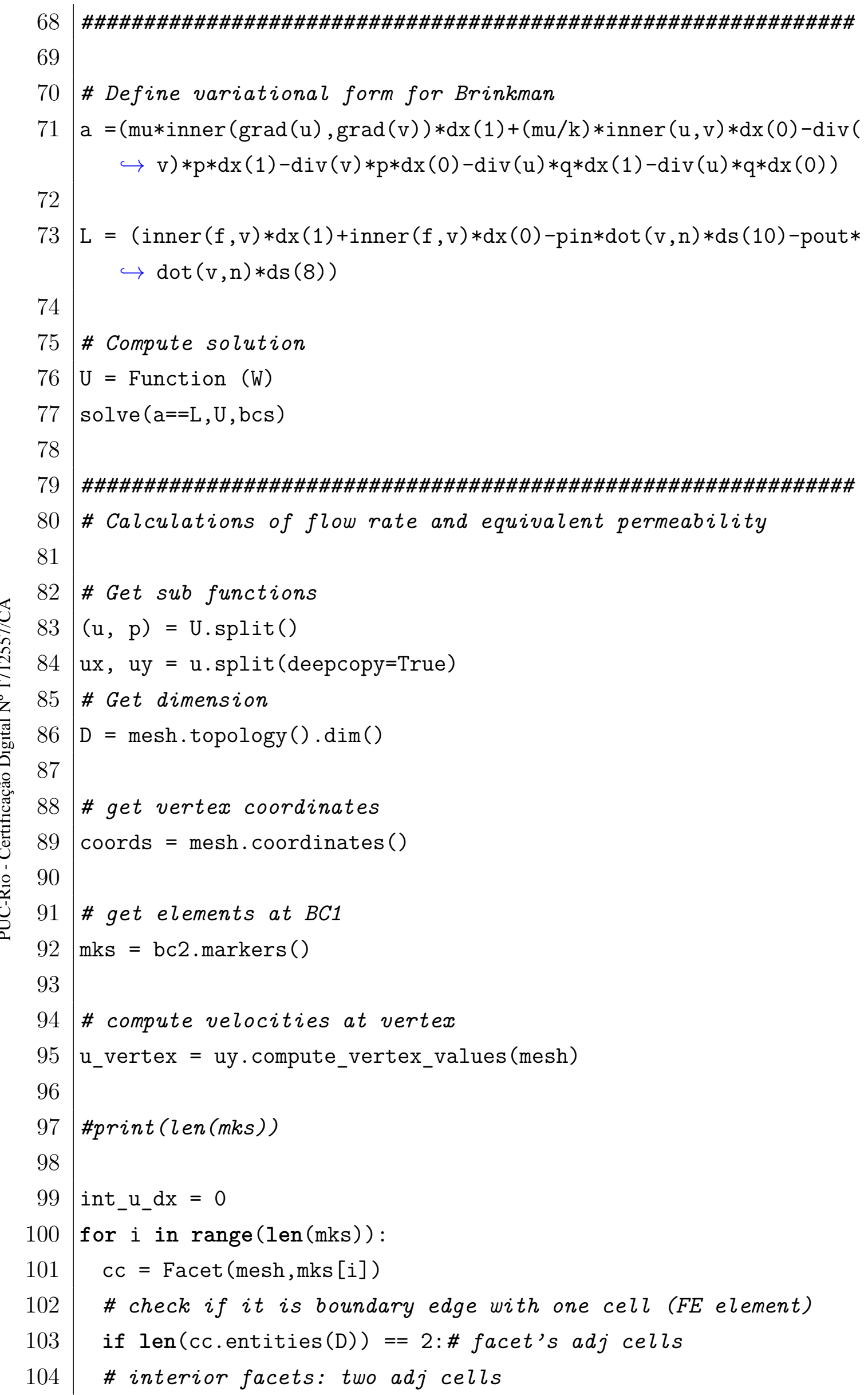




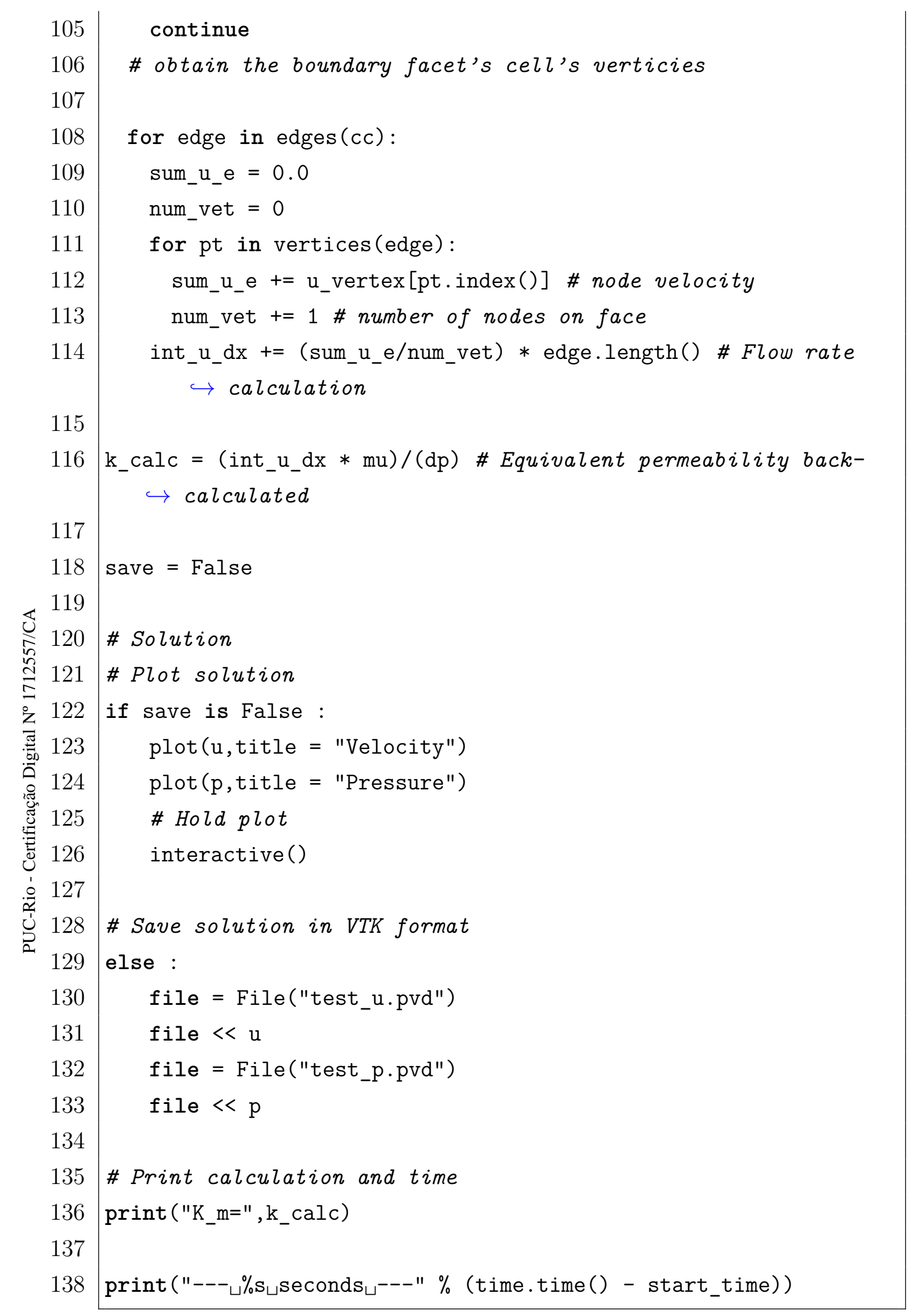


Scripts A.2: Finite element mesh

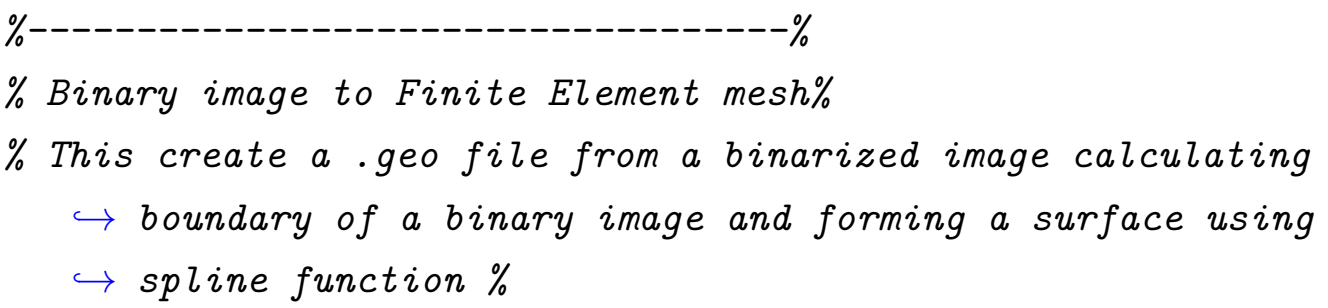




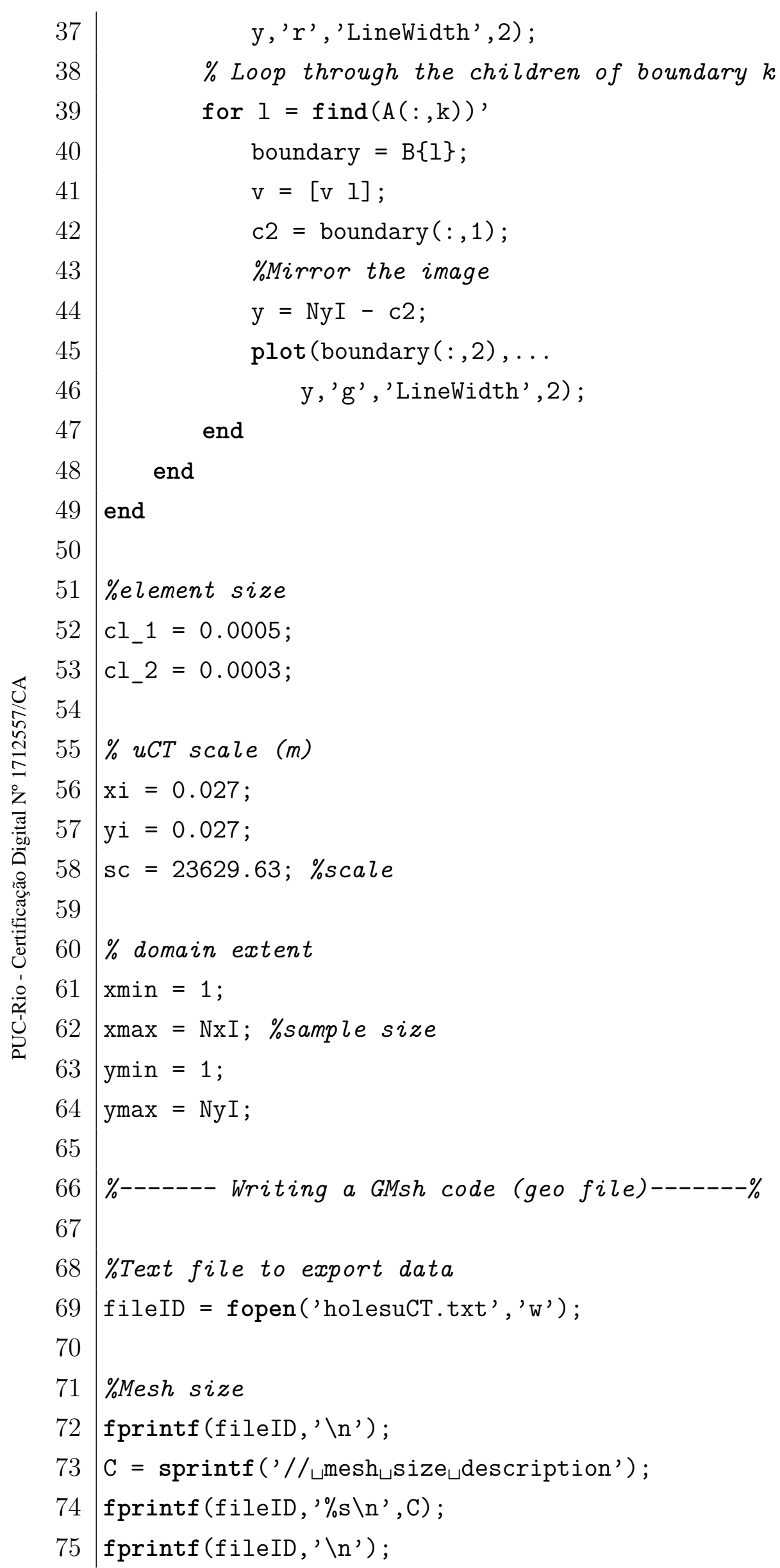




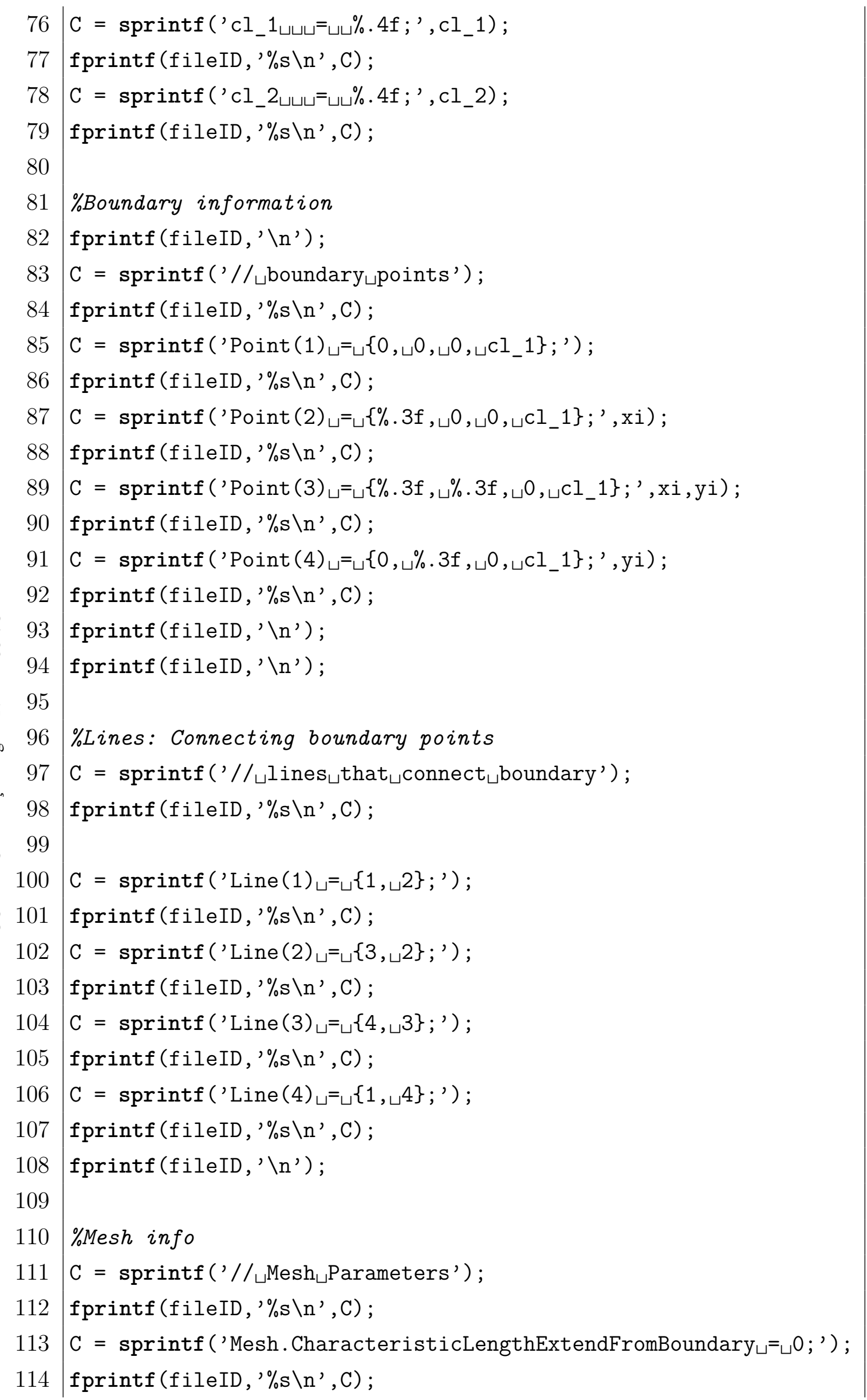




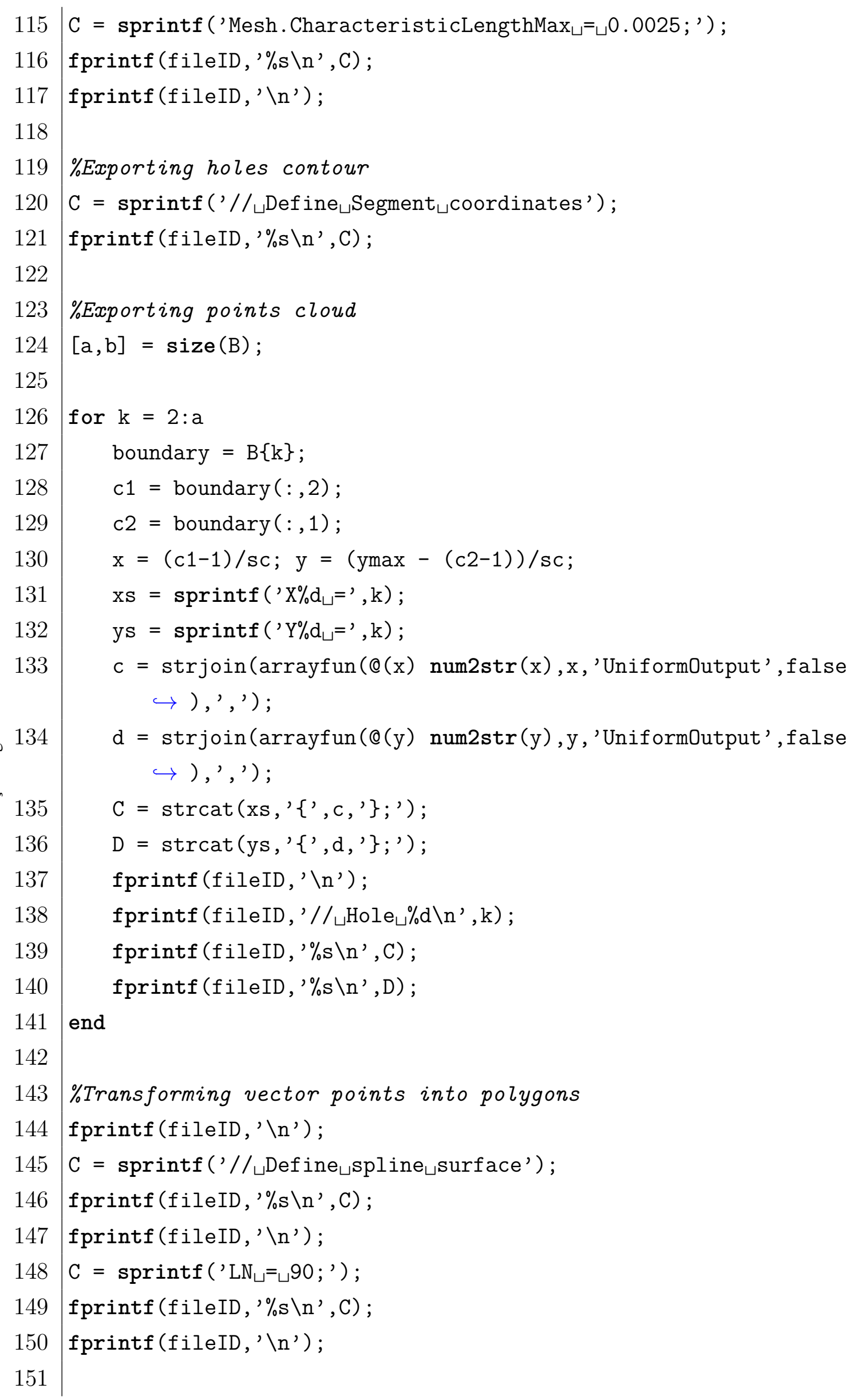




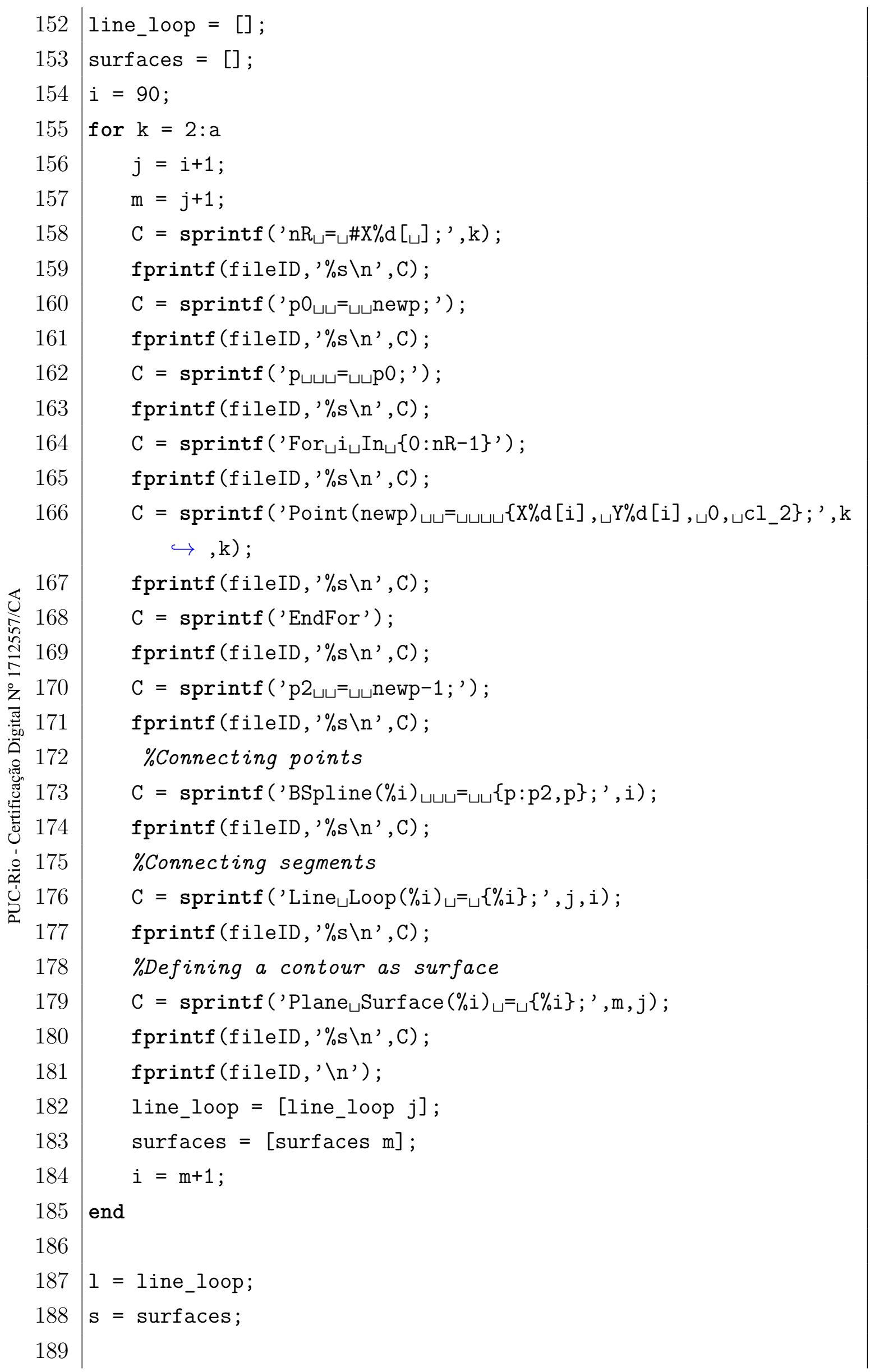




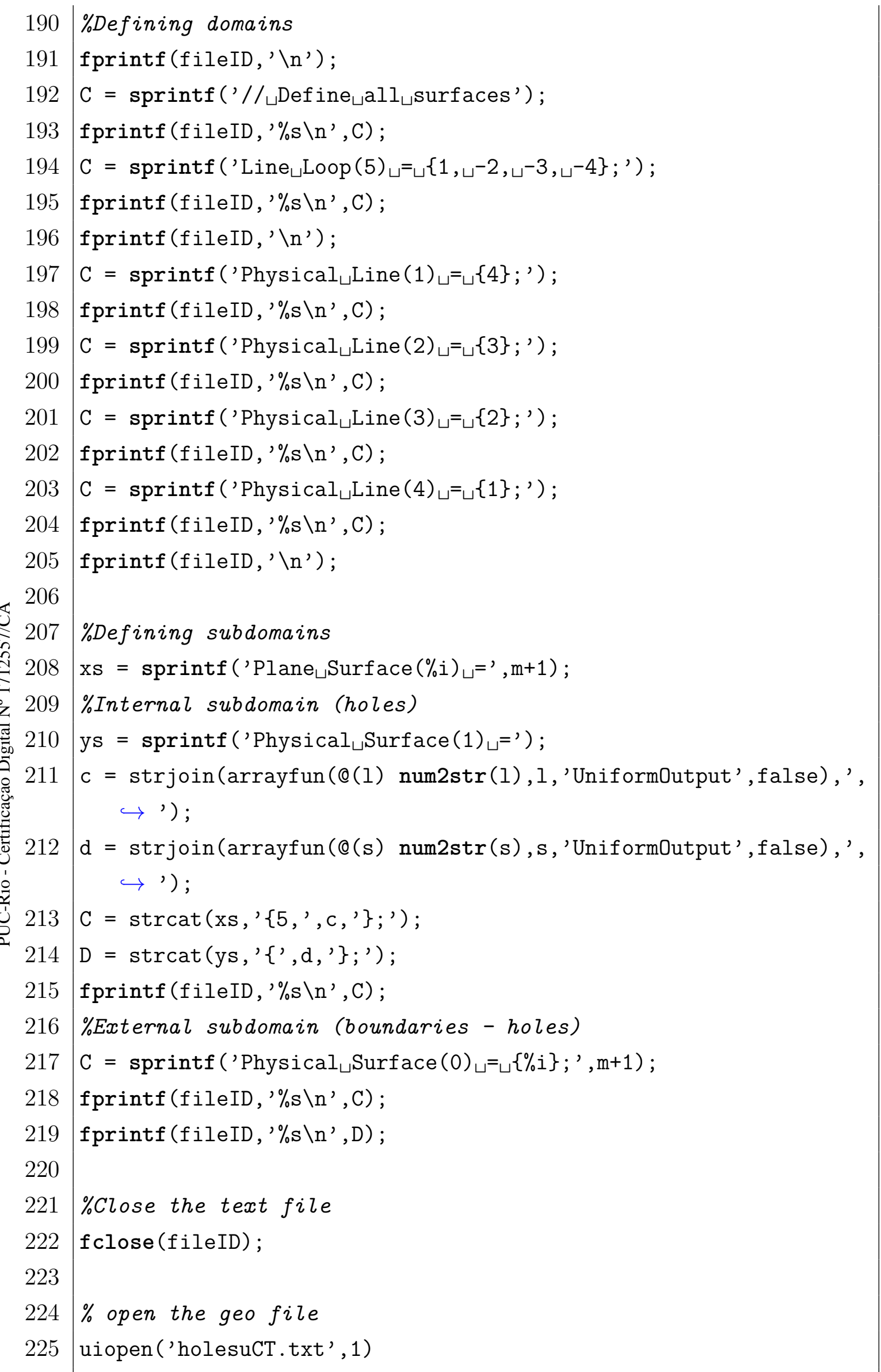

